\title{
MEMBERS OF THE SOCIETY
}

\author{
September, 1942
}

This directory includes the name of every person who is a member of the Society as of September, 1942.

Personal information is as follows: (a) surname; (b) initials or one name with parentheses around parts usually omitted in correspondence; first names of women members are written out; (c) official title or position; (d) name and address of institution or organization; (e) mail address if different from the institution address; (f) in italic type, information regarding temporary or special assignments.

For many members in foreign countries, it has been necessary to repeat information which appeared in the previous directory.

The $\dagger$ before a name indicates life membership.

The $\S$ indicates membership as a nominee of an institutional member.

Ablow, C. M. 1943 N. Van Ness Ave., Los Angeles, Calif. Ensign U.S.N.R., Naval Base, Long Beach, Calif.

Abramowitz, Milton. Asso. Mathematician, Mathematical Tables Project, National Bureau of Standards, U.S. Dept of Commerce, 70 Columbus Ave., New York, N.Y. 2775 Kingsbridge Ter., Bronx.

Adams, C. R. Prof., Brown Univ., Providence, R.I. 60 Intervale Road.

Adams, E. P. Prof., Princeton Univ., Princeton, N.J. Edgerstoune.

Adams, G. J. Junior Civil Engineer, State Highway Dept., Linden, Ala. 2411 14th Ave., Sheffield, Ala.

Adams, Dr. Rachel B. (Mrs. C. R.). Radcliffe Coll., Cambridge, Mass. 60 Intervale Road, Providence, R.I.

Ader, O. B. Asst. Prof., Wofford Coll., Spartanburg, S.C. 492 N. Church St.

Adkisson, V. W. Prof., Univ. of Arkansas, Fayetteville, Ark. 236 Buchanan St. Adler, Dr. Claire F. (Mrs. E. H.). 189-21 Tioga Dr., St. Albans, N.Y.

§Adler, F. T. Visiting Asst. Prof., Physics Dept., Univ. of Wisconsin, Madison, Wis.

Adshead, J. G. Asso. Prof., Dalhousie Univ., Halifax, N.S., Canada.

Agard, H. L. Prof., Williams Coll., Williamstown, Mass.

Agnew, R. P. Prof., Cornell Univ., Ithaca, N.Y. White Hall.

Alaoglu, Dr. Leonidas. Instr., Purdue Univ., Lafayette, Ind.

Albert, A. A. Prof., Univ. of Chicago, Chicago, I1l. Eckhart Hall.

Albert, Dr. G. E. Instr., Ohio State Univ., Columbus, Ohio. University Hall.

Albert, O. W. Prof., Univ. of Redlands, Redlands, Calif. 629 Buena Vista St.

Alden, H. H. Prof., New Mexico Military Inst., Roswell, N.Mex. Box 1237.

Alexander, J. W. Prof., Inst. for Advanced Study, Princeton, N.J. 29 Cleveland Lane.

Alfieri, F. A. Metropolitan Life Ins. Co., 1 Madison Ave., New York, N.Y. 9 Metropolitan Oval, Bronx.

Alger, P. L. Staff Asst., General Electric Co., Schenectady, N.Y. 1758 Wendell Ave.

Allen, E. B. Prof., Rensselaer Polytech. Inst., Troy, N.Y. 4 Sheldon Ave.

Allen, E. S. Asso. Prof., Iowa State Coll., Ames, Iowa.

Allen, Dr. Florence E. Instr., Univ. of Wisconsin, Madison, Wis. 219 Lathrop St.

Allen, Harriet W. Asst. Prof., Hollins Coll., Hollins College, Va. Physicist, Bureau of Ordnance, U. S. Navy Dept., Washington, D.C. 2141 Eye St. N.W.

Allendoerfer, C. B. Asso. Prof., Haverford Coll., Haverford, Pa. Asso. Physicist, U. S. Navy Dept., Washington, D.C. 8603 Hempstead Ave., Bethesda, Md.

Alsberg, Julius. Consulting Engineer. Office of Chief of Chemical Warfare Service, U. S. War Dept., Washington, D.C. 2515 K St. N.W.

§Alton, T. P. Asst., Univ. of I1linois, Urbana, I11. 153 Math. Bldg.

Ambrose, Dr. Warren. Instr., Princeton Univ., Princeton, N.J. Fine Hall.

Ames, D. B. Asst. Prof., Rensselaer Polytech. Inst., Troy, N.Y. 117 Elmgrove Ave.

Ames, L. D. Prof., Univ. of Southern California, Los Angeles, Calif. 
Amundson, N. R. Instr., Univ. of Minnesota, Minneapolis, Minn. 208 Main Engineering Bldg.

Ananda-Rau, K. Prof., Presidency Coll., Madras, India.

Anastasia Maria, Sister. Prof., Immaculata Coll., Immaculata, $\mathrm{Pa}$.

Andersen, C. C. 1917 Warren Blvd., Chicago, Ill. 2d Lt., U.S.A., Air Corps, Morris Field, Charlotte, N.C.

Anderson, Mae R. Prof., Concordia Coll., Moorhead, Minn. 507 10th St. S.

Anderson, Dr. P. H. Instr., Louisiana State Univ., University, La.

Anderson, R. Lucile. Asst. Prof., Hunter Coll., 695 Park Ave., New York, N.Y.

Anderson, T. W. Instr., Princeton Univ., Princeton, N.J. 2435 Laundale Ave., Evanston, I11.

Anderson, W. E. Prof., Miami Univ., Oxford, Ohio. 112 E. Walnut St.

§Annenberg, Lawrence. 131 E. 21st St., Brooklyn, N.Y.

Anthony, Lucius. Woodinville, Wash.

Archibald, H. F. Asso. Prof., Keuka Coll., Keuka Park, N.Y.

†Archibald, R. C. Prof., Brown Univ., Providence, R.I.

Archibald, R. G. Asst. Prof., Queens Coll., Flushing, N.Y.

Arms, R. A. Prof., Gettysburg Coll., Gettysburg, $\mathrm{Pa} .59$ Lincoln.

Armstrong, Dr. Beulah M. Asso., Univ. of Illinois, Urbana, I11. 364 Math. Bldg.

Arnold, Dr. B. H. Instr., Purdue Univ., Lafayette, Ind.

Arnold, Dr. H. A. 2480 Ĺake St., Lincoln, Neb. Lt. (j.g.), U.S.N.R

Arnold, H. E. Asso. Prof., Wesleyan Univ., Middletown, Conn. Wesleyan Station.

†Arnold, J. W. Lt. Col., U.S.A., Coast Artillery Corps, Camp Haan, Calif.

\$Arnold, Dr. K. J. Instr., Massachusetts Inst. of Tech., Cambridge, Mass.

Arnoldy, Sister M. Nicholas. Marymount Coll., Salina, Kan.

Aroian, Dr. L. A. Instr., Hunter Coll., Bedford Park Blvd. and Navy Ave., New York, N.Y.

Arrow, K. J. Fellow, Economics Dept., Columbia Univ., New York, N.Y. 749 West End Ave.

Arthur, R. S. Univ. of California at Los Angeles, Los Angeles, Calif. 314 Buena Vista St., Redlands, Calif.

Artin, Emil. Prof., Indiana Univ., Bloomington, Ind. 715 S. Fess Ave.

Ashman, E. T. Darien High School, Darien, Conn. 11 Fairview Ave.

Assadourian, Dr. Fred. Instr., New York Univ., University Heights, New York, N.Y.

Astrachan, Max. Asso. Prof., Antioch Coll., Yellow Springs, Ohio.

Atanasoff, J. V. Asso. Prof., Iowa State Coll., Ames, Iowa. Physics Bldg. Official Investigator, National Defense Research Com.

§Atchison, W. F. Asst., Univ. of Illinois, Urbana, I11. 153 Math. Bldg.

\$Atkins, H. P. Instr., Univ. of Rochester, Rochester, N.Y. River Campus.

\$Avann, Dr. S. P. Instr., Washington Univ., St. Louis, Mo.

Ayres, Frank. Asso. Prof., Dickinson Coll., Carlisle, Pa. 426 S. Pitt St.

Ayres, H. C. Asst. Prof., U. S. Naval Acad., Annapolis, Md. Lt., U.S.N.R., U.S.S. Texas, Care of Postmaster, New York, N.Y.

Ayres, w. L. Prof., Purdue Univ., Lafayette, Ind.

Babcock, R. W. Dean, School of Arts and Sciences, Kansas State Coll. of Agric. and Applied Science, Manhattan, Kan.

Babcock, Wealthy. Asso. Prof., Univ. of Kansas, Lawrence, Kan.

†Bacon, Clara L. Prof. Emeritus, Goucher Coll., Baltimore, Md. 2316 N. Calvert St.

Bacon, H. M. Asst. Prof., Stanford Univ., Stanford University, Calif. Box 1144.

Baer, Reinhold. Asso. Prof., Univ. of Illinois, Urbana, Ill.

Bagby, L. C. Educational Dir., Aviation School, Hartung Aircraft Corp., Detroit, Mich. 7027 E. Warren.

Baidaff, B. I. Prof., Univ. of Buenos Aires, Buenos Aires, Argentina. Av. de Mayo 560 .

Bailey, H. W. Dir., Personnel Bureau, and Asso. Prof., Univ. of Illinois, Urbana, III.

Bailey, R. P. Asst. Prof., Lafayette Coll., Easton, Pa. 628 Monroe St.

Baker, Frances E. Asso. Prof., Vassar Coll., Poughkeepsie, N.Y. 
Baker, Dr. G. A. Instr. and Junior Statistician, Coll. of Agric., Univ. of California, Davis, Calif.

Bal, Y. K. Columbia Univ., New York, N.Y. 500 Riverside Dr.

Ball, N. H. Asst. Prof., U.S. Naval Acad., Annapolis, Md.

Ballantine, Dr. Constance R. (Mrs. J. P.). Asso., Univ. of Washington, Seattle, Wash. 1802 Ravenna Blvd.

Ballantine, J. P. Prof., Univ, of Washington, Seattle, Wash. 1802 Ravenna Blvd.

Ballard, Dr. Ruth M. (Mrs. F. K.). Wright Junior Coll., Chicago, I11. 636 Wellington Ave.

Ballou, D. H. Asst. Prof., Middlebury Coll., Middlebury, Vt. 18 S. Pleasant St.

Bamforth, F. R. Asso. Prof., Ohio State Univ., Columbus, Ohio.

Bankier, Dr. J. D. Instr., Johns Hopkins Univ., Baltimore, Md.

Banks, G. B. Prof., Niagara Univ., Niagara University, N.Y.

Baños, Alfredo. Dir., Inst. of Physics, and Prof., Univ. of Mexico, Tacuba 5, Mexico, D.F., Mexico.

§Barankin, E. W. Asst., Univ. of California, Berkeley, Calif. 2540 LeConte Ave.

Barber, Dr. S. F. Instr., Coll. of the City of New York, 139th St. and Convent Ave., New York, N.Y. Instr., Defense Training Inst., 375 Pearl St., Brooklyn, N.Y.

Bardell, R. H. Asst. Prof. and Acting Asst. Dir., Univ. of Wisconsin at Milwaukee, Milwaukee, Wis.

Bareis, Grace M. Asst. Prof., Ohio State Univ., Columbus, Ohio. Canal Winchester, Ohio.

§Bargmann, Dr. Valentin. Asst., Inst. for Advanced Study, Princeton, N.J.

Barker, J. E. Asso. Mathematician, U.S. Naval Proving Ground, Dahlgren, Va.

Barlaz, Joshua. Instr., Coll. of Engineering and Commerce, Univ. of Cincinnati, Cincinnati, Ohio.

Barnard, R. W. Asso. Prof., Univ. of Chicago, Chicago, Ill. Eckhart Hall.

Barnes, J. L. Prof., Tufts Coll., Medford, Mass. 41 Harold Ave., Princeton, N.J.

Barnett, I. A. Asso. Prof., Univ. of Cincinnati, Cincinnati, Ohio.

Barnett, Joseph. Asst. Prof., Oklahoma Agric. and Mech. Coll., Stillwater, Okla. 520 Maple Ave.

Baron, Louis. Instr., Washington Square Coll., New York Univ., New York, N.Y.

Barral-Souto, José. Prof., Univ. of Buenos Aires, Buenos Aires, Argentina. Córdoba 1459.

Barron, J. J. Asso. Prof., St. Joseph Coll., West Hartford, Conn.

Barrow, D. F. Prof., Univ. of Georgia, Athens, Ga. 260 Cherokee Ave.

Barta, V. P. Research Physicist, Massachusetts Inst. of Tech., Cambridge, Mass. 247 N. 11th Ave., Corvallis, Ore.

Bartels, Dr. R. C. F. Instr., Univ. of Michigan, Ann Arbor, Mich. Engineer, Structures Section, Bureau of Aeronautics, U. S. Navy Dept., Washington, D.C.

Barten, H. J. Johns Hopkins Univ., Baltimore, Md. 1518 Moreland Ave.

Bartky, Walter. Asso. Dean of Physical Sciences and Prof., Univ. of Chicago, Chicago, I11.

Bartleson, E. L. Asst. Mathematician, Actuarial Dept. General, Prudential Ins. Co. of America, Newark, N.J.

Basch, Dr. A. E. Coll. of the City of New York, New York, N.Y. 503 W. 121st St.

Basoco, M. A. Prof., Univ. of Nebraska, Lincoln, Neb.

§Bassford, H. R. Actuary, Metropolitan Life Ins. Co., 1 Madison Ave., New York, N.Y.

Basye, Dr. R. E. 5529 Brooklyn Ave., Kansas City, Mo. Lt. (j.g.), U.S.N.R.

Batchelder, P. M. Asso. Prof., Univ. of Texas, Austin, Tex. 910 W. $22 \mathrm{~d}$ St.

Bateman, Harry. Prof., California Inst. of Tech., Pasadena, Calif.

Bateman, P. T. 421 Lawnton Road, Willow Grove, Pa.

Bates, O. K. Prof., St. Lawrence Univ., Canton, N.Y. 44 E. Main St.

†Battig, Leon. Instr., Univ. of Wisconsin at Milwaukee, Milwaukee, Wis. 2535 Elizabeth St., Sheboygan, Wis.

Bauer, P. S. Vice Pres., North Shore News Co., 50 Exchange St., Lynn, Mass.

Baumgart, J. K. 919 Adams St., Maywood, I1l. Instr., U.S.A., Air Corps Tech. School, Madison, Wis.

Beal, F. W. Prof., Univ. of Pennsylvania, Philadelphia, Pa. College Hall. 
Beale, F. S. Asst. Prof., Lehigh Univ., Bethlehem, Pa. 316 W. Packer Ave. Beamish, Sister M. Amator. 5601 Kingsessing Ave., Philadelphia, Pa.

Bearman, J. E. 1811 Queen Ave. N., Minneapolis, Minn. U.S.A., Coast Artillery Replacement Training Center, Fort Eustis, Va. Care of H. Nisselson, 1630 $R$ St. N.W., Washington, D.C.

Beatley, Ralph. Asso. Prof., Graduate School of Education, Harvard Univ., Cambridge, Mass.

Beatty, Samuel. Dean, Faculty of Arts, Univ. of Toronto, Toronto, Ont., Canada.

Beaty, Dr. Marjorie H. (Mrs. D. W.). 717 E. Main St., Vermillion, S.Dak.

Beaumont, Dr. R. A. Instr., Univ. of Washington, Seattle, Wash.

Beaver, R. A. Asst. Prof., New York State Coll. for Teachers, Albany, N.Y.

Beckenbach, E. F. Asst. Prof., Univ. of Michigan, Ann Arbor, Mich.

Becker, F. A. Instr., St. Mary's Univ., San Antonio, Tex.

Becker, Dr. Miriam F. Care of A. Mazur, 65 Park Ter. E., New York, N.Y.

Beckwith, Ethelwynn R. (Mrs. W. E.). Prof., Milwaukee-Downer Coll., Milwaukee, Wis.

Beckwith, W. S. Asso. Prof., Univ, of Georgia, Athens, Ga.

Beegle, B. L. Dean and Prof,, Seattle Pacific Coll., Seattle, Wash.

Beenken, May M. Prof., State Teachers Coll., Oshkosh, Wis.

Beesley, E. M. Asst. Prof., Univ. of Nevada, Reno, Nev.

Belcher, D. R. Asst. Chief Statistician, American Telephone and Telegraph Co., 195 Broadway, New York, N.Y.

Bell, Clifford. Asso. Prof., Univ. of California at Los Angeles, Los Angeles, Calif. 10514 Rochester Ave.

Bell, E. T. Prof., California Inst. of Tech., Pasadena, Calif.

$\S$ Bell, J. C. Asst., Univ. of Illinois, Urbana, I11. 160 Math. Bldg.

Bell, Dr. J. H. Research Asst., National Research Council, Ottawa, Ont., Canada. 95 Barker St., London, Ont., Canada.

Bell, Lois E. Instr., Independence Junior Coll., Independence, Kan. Box 83.

Bell, P. O. Asst. Prof., Univ. of Kansas, Lawrence, Kan. 2201 New Hampshire St.

Bellamy, B. C. Civil Engineer. Lamont, Wyo.

§Bellman, Richard. No address.

Benac, Dr. T. J. Instr., U.S. Naval Acad., Annapolis, Md.

Bender, J. R. Asso. Prof., Univ. of Idaho, Moscow, Idaho.

†Bennett, A. A. Prof., Brown Univ., Providence, R.I.

Bennett, B. M. Columbia Univ., New York, N.Y. Asst. Mathematician, U.S. Naval Proving Ground, Dahlgren, Va. $1410 M \cdot S t$. N.W., Washington, D.C.

Bennett, Theodore. Asst. Prof., Marietta Coll., Marietta, Ohio.

Benscoter, S. U. Asst. Engineer, U.S. Engineer Office, Vicksburg, Miss.

§Benson, Kathryn E. Asst., Univ. of California, Berkeley, Calif.

Bentley, Dr. A. F. R.F.D. 2, Paoli, Ind.

$\S$ Bentz, R. P. Instr., Pennsylvania State Coll., State College, Pa.

Berg, Dr. W. D. Instr., State Univ. of Iowa, Iowa City, Iowa. 211 Physics Bldg.

Bergman, Dr. Stefan. School of Mech., Brown Univ., Providence, R.I.

Bergmann, P. G. Asst. Prof., Physics Dept., Lehigh University, Bethlehem, Pa.

$\S$ Bernard, R. R. Univ. of Virginia, University Station, Charlottesville, Va.

Bernstein, B. A. Prof., Univ. of California, Berkeley, Calif.

Bernstein, Dr. Dorothy L. Instr. and Research Asso., Univ. of California, Berkeley, Calif.

Bernstein, Felix. Prof., New York Univ., 477 1st Ave., New York, N.Y.

Bernstorf, Ella E. Dean, State Teachers Coll., California, Pa.

Berry, A. C. Asso. Prof., Lawrence Coll., Appleton, Wis.

Berry, W. J. Prof., Polytech. Inst. of Brooklyn, Brooklyn, N.Y. 224 St. John's P1.

Berwick, W. E. H. Prof. Emeritus, University Coll. of North Wales, Bangor, North Wales. Care of E. G. Phillips.

Besancon, R. M. Coll. of Pharmacy, Univ. of Illinois, Chicago, I11. $2 d$ Lt., A.U.S.

Beth, R. A. Asso. Prof., Michigan State Coll., East Lansing, Mich. Consultant, National Defense Research Com.; Physicist, Com. on Passive Protection Against Bombing. Palmer Physics Lab., Princeton Uniw., Princeton, N.J. 
Betz, Dr. E. E. Instr., U.S. Naval Acad., Annapolis, Md. 1106 Maple St. Lt. (j.g.), U.S.N.R.

Betz, Herman. Asst. Prof., Univ. of Missouri, Columbia, Mo.

Betz, Dr. William. Mathematics Specialist, Public Schools of Rochester, Rochester, N.Y. 652 Melville St.

Beveridge, H. R. Prof., Monmouth Coll., Monmouth, I11. 1043 E. Detroit Ave.

Bibb, S. F. Asso. Prof., Illinois Inst. of Tech., Chicago, I11.

Biesele, Dr. F. C. Instr., Univ. of Utah, Salt Lake City, Utah.

Bilgery, Conrad. Prof., Regis Coll., Denver, Colo.

Biot, M. A. Asst. Prof., Columbia Univ., New York, N.Y. Research Asso. in Aeronautics, California Inst. of Tech., Pasadena, Calif.

Birchby, W. N. Asst. Prof., California Inst. of Tech., Pasadena, Calif.

Birchenough, Harry. Prof., New York State Coll. for Teachers, Albany, N.Y.

Bird, M. T. Asst. Prof., Utah State Agric. Coll., Logan, Utah.

Birkhoff, Garrett. Asso. Prof., Harvard Univ., Cambridge, Mass. 45 Fayerweather St.

Birkhoff, G. D. Prof., Harvard Univ., Cambridge, Mass. 987 Memorial Dr.

Birnbaum, Z. W. Asst. Prof., Univ. of Washington, Seattle, Wash.

Bisshopp, K. E. 1136 LaSalle St., Beloit, Wis.

§Bissinger, B. H. Instr., Cornell Univ., Ithaca, N.Y.

Black, A. H. Prof., Lebanon Valley Coll., Annville, Pa. 485 Maple St.

Black, Florence L. Asso. Prof., Univ. of Kansas, Lawrence, Kan.

Black, H. L. Prof., Westminster Coll., New Wilmington, Pa. $200 \mathrm{High} \mathrm{St.}$

Blackall, Dr. C. J. North Baltimore, Ohio. U.S.A., Camp Wallace, Tex.

Blackwell, Dr. David. Instr., Southern Univ., Scotlandville, La.

Blake, Dr. Archie. Engineer, National Inventors Council, Washington, D.C. 2812 Cathedral Ave. N.W.

Blake, Dr. E. M. Drawer A, Pratt Station, Brooklyn, N.Y.

Blake, R. G. Hernando High School, Brooksville, Fla. 303 E. Jefferson St.

Blanc, Charles. Prof., Univ. of Lausanne, Lausanne, Switzerland. Ave. Jolimont 13.

Blanch, Dr. Gertrude. Asso. Mathematician, Mathematical Tables Project, National Bureau of Standards, U.S. Dept. of Commerce, 70 Columbus Ave., New York, N.Y. 4823 Clarendon Road, Brooklyn, N.Y.

Blanche, Dr. E. E. Instr., Michigan State Coll., East Lansing, Mich.

§Blankinship, W. A. Industrial Mathematician, U.S. Rubber Co., Detroit, Mich. 5026 Chalmers Ave.

Blaquier, Juan. Prof., Univ. of Buenos Aires, Buenos Aires, Argentina. Arroyo 1000.

Blau, J. H. Ordnance Lab., Navy Yard, U.S. Navy Dept., Washington, D.C.

Bleick, Dr. W. E. Instr., U. S. Naval Acad., Annapolis, Md. 114 Monticello Ave. Lt., U.S.N.R., Bureau of Ordnance, U.S. Navy Dept., Washington, D.C.

Blichfeldt, H. F. Prof. Emeritus, Stanford Univ., Stanford University, Calif. 520 W. Crescent Dr., Palo Alto, Calif.

†Bliss, G. A. Prof. Emeritus, Univ. of Chicago, Chicago, I11.

Blumberg, Henry. Prof., Ohio State Univ., Columbus, Ohio. 76 E. Blake Ave.

Blumenthal, L. M. Asso. Prof., Univ. of Missouri, Columbia, Mo.

Boas, Mary L. (Mrs. R. P.). Instr., Duke Univ., Durham, N.C. 2708 Legion Ave.

Boas, Dr. R. P. 2708 Legion Ave., Durham, N.C. Junior Ground School Instr., U.S.N. Pre-Flight School, Chapel Hill, N.C.

Bobonis, Augusto. Asso. Prof., Univ. of Puerto Rico, Rio Piedras, Puerto Rico.

Bochner, Salomon. Asso. Prof., Princeton Univ., Princeton, N.J. Fine Hall.

Bode, Dr. H. W. Bell Telephone Labs., 463 West St., New York, N.Y.

Boeder, Dr. Paul. American Optical Co., Southbridge, Mass.

Boehm, Frank. Manager, Life Ins. Dept., 80 Maiden Lane, New York, N.Y.

Boeker, Mary I. D. (Mrs.). Instr., Brooklyn Coll., Brooklyn, N.Y. Collingwood Hotel, 45 W. 35th St., New York, N.Y.

Boggs, Dr. Elizabeth M. (Mrs. F. W.). Lecturer, Physics Dept., Univ. of Pittsburgh, Pittsburgh, Pa.

Bohnenblust, H. F. Asso. Prof., Princeton Univ., Princeton, N.J. 
Bohr, H. A. Prof., Univ. of Copenhagen, Copenhagen, Denmark. Maglevaenget 9, Charlottenlund, Denmark.

Boldyreff, A. W. Asso. Prof., Univ. of Arizona, Tucson, Ariz. 2309 E. 5th St.

Bolza, Oskar. Prof., Univ. of Freiburg, Freiburg i. Br., Germany. Hotel Ochsen, Tubingen, Germany.

Bond, W. M. Instr., Polytech. Inst. of Brooklyn, Brooklyn, N.Y.

Borgman, W. M. Asst. Prof., Wayne Univ., Detroit, Mich.

Borofsky, Samuel. Asst. Prof., Brooklyn Coll., Brooklyn, N.Y. 245 Lenox Road.

Boswell, J. M. Prof., Cumberland Coll., Williamsburg, Ky. Lt., U.S.N.R.

Botts, T. A. Fellow, Univ. of Virginia, University Station, Charlottesville, Va.

Bouckaert, Dr. L. P. 112 Rue Christine, Ostend, Belgium.

Bourgin, D. G. Asst. Prof., Univ. of Illinois, Urbana, I1l.

§Bourne, S. G. Johns Hopkins Univ., Baltimore, Md.

Bowden, Joseph. Prof. Emeritus, Adelphi Coll., Garden City, N.Y. 21 Carleton P1., Baldwin, N.Y.

Bowden, Muriel. 405 Park Ave., New York, N.Y.

Bower, Julia W. Asso. Prof., Connecticut Coll., New London, Conn.

Bower, Dr. O. K. Instr., Univ. of Illinois, Urbana, I11. 369 Math. Bldg.

§Bowie, Clagett. Johns Hopkins Univ., Baltimore, Md. Rowland Hall.

Bowie, H. E. Prof., American International Coll., Springfield, Mass. 14 Gunn Sq. Boyce, M. G. Asst. Prof., Western Reserve Univ., Cleveland, Ohio.

Boyd, P. P. Dean, Coll. of Arts and Sciences, Univ. of Kentucky, Lexington, Ky. Boyer, C. B. Asst. Prof., Brooklyn Coll., Brooklyn, N.Y.

Bradley, A. D. Asst. Prof., Hunter Coll., Bedford Park Blvd. and Navy Ave., New York, N.Y. 66 Villard Ave., Hastings-on-Hudson, N.Y.

$\S$ Bradshaw, C. H. Johns Hopkins Univ., Baltimore, Md.

Bradshaw, J. W. Prof., Univ. of Michigan, Ann Arbor, Mich. 1304 Cambridge Road.

Bradt, Paul. Physicist, National Bureau of Standards, U.S. Dept. of Commerce, Washington, D.C. 1847 Mintwood P1. N.W.

Brady, Dr. C. P. Los Angeles City Coll., Los Angeles, Calif. Lt., U.S.N.R., U. S. Naval Acad., Annapolis, Md. Box 232 A, Fairfax Road, R.F.D. 1.

$\S$ Brady, G. V. Metropolitan Life Ins. Co., 180 Wellington St., Ottawa, Ont., Canada.

Brady, Mary L. Asst. Prof., Coll. of Mt. St. Vincent, New York, N.Y.

Brahana, H. R. Prof., Univ. of Illinois, Urbana, I1l. 707 S. McCullough.

Brainard, Millar. 17220 Aldersyde Dr., Shaker Heights, Cleveland, Ohio.

Bramble, C. C. Prof., Postgraduate School, U. S. Naval Acad., Annapolis, Md. 145 Monticello Ave.

Brand, Louis. Prof., Univ. of Cincinnati, Cincinnati, Ohio. Coordinator of Research in Math. and Mech., Wright Field, Dayton, Ohio.

Brandeberry, J. B. Prof., Univ. of Toledo, Toledo, Ohio.

Branson, H. R. Asst. Prof., Howard Univ., Washington, D.C. Chemistry B1dg.

Brant, Dr. Laura. 33 Capwell Ave., West Warwick, R.I.

Brasefield, S. E. Prof., Rutgers Univ., New Brunswick, N.J.

Bratton, W. A. Prof., Whitman Coll., Walla Walla, Wash.

§Brauer, A. T. Asst. Prof., Univ. of North Carolina, Chapel Hill, N.C. 408 North St.

Brauer, Richard. Asst. Prof., Univ. of Toronto, Toronto, Ont., Canada.

Bray, H. E. Prof., Rice Inst., Houston, Tex.

Breit, Gregory. Prof., Physics Dept., Univ. of Wisconsin, Madison, Wis. Sterling Hall.

Brenke, W. C. Prof., Univ. of Nebraska, Lincoln, Neb. 1250 S. 21st St.

Brennan, J. F. 1769 Broadway, San Francisco, Calif. Lt., U.S.N.R.

Brenner, Dr. J. L. 2431 N. Underwood St., East Falls Church, Arlington, Va.

Brewer, B. W. Asst. Prof., Agric. and Mech. Coll. of Texas, College Station, Tex. 17 S. Bluff St., Janesville, Wis. Lt. (j.g.), U.S.N.R.

Briant, R. C. Fellow, Mellon Inst., Pittsburgh, Pa. 403 Glasgow Road.

§Bridgman, P. W. Prof., Harvard Univ., Cambridge, Mass. Research Physics Lab.

Bright, S. K. Instr., Angleton Public Schools, Angleton, Tex. 
Brink, R. W. Prof., Univ. of Minnesota, Minneapolis, Minn. 2243 Hoyt Ave., St. Paul, Minn.

Brinkmann, H. W. Asso. Prof., Swarthmore Coll., Swarthmore, Pa.

Bristow, Dr. Leonard. Instr., Univ. of Illinois, Urbana, I1l. 360 Math. Bldg.

Britton, J. R. Asst. Prof., Univ. of Colorado, Boulder, Colo.

Brixey, J. C. Asso. Prof., Univ. of Oklahoma, Norman, Okla. 927 S. Pickard.

Brodie, Helen C. Fellow, Coll. of the City of New York, New York, N.Y. 29 Washington Sq. W.

Brooks, F. L. Asst. Prof., Kent State Univ., Kent, Ohio. Box 224.

Brothers, W. H. Asso. Prof., Talladega Coll., Talladega, Ala.

Brown, Dr. A. A. F. Research Dept., L. Bamberger and Co., Newark, N.J. 11 Edgehill St., Princeton, N.J.

Brown, A. B. Asst. Prof., Queens Coll., Flushing, N.Y.

Brown, B. H. Prof., Dartmouth Coll., Hanover, N.H. 7 Ripley Road.

Brown, B. L. Asso. Prof., Amherst Coll., Amherst, Mass. 19 Hitchcock Road.

Brown, Dr. F. W. Asso. Physicist, Bureau of Mines, U.S. Dept. of Interior, Bruceton, $\mathrm{Pa}$.

Brown, Dr. G. W. Research Asst., Princeton Univ., Princeton, N.J. 172 Nassau St.

Brown, Dr. H. K. Instr., Rensselaer Polytech. Inst., Troy, N.Y. 703 Grand St.

Brown, Myrtle C. Asso. Prof., North Texas State Teachers Coll., Denton, Tex. 5334 Teachers College Station.

Brown, O. E. Asso. Prof., Case School of Applied Science, Cleveland, Ohio. 1842 Taylor Road, East Cleveland, Ohio.

Brown, R. D. Engineer, Patent Dept., Philco Corp., Philadelphia, Pa. Blue Bell, Montgomery Co., Pa.

Brown, T. H., Prof., Graduate School of Business Administration, Harvard Univ., Soldiers Field, Boston, Mass.

Browne, E. T. Prof., Univ. of North Carolina, Chapel Hill, N.C. 730 E. Franklin St.

Bruce, R. E. Prof., Boston Univ., Boston, Mass.

Bruck, Dr. R. H. Instr., Univ. of Wisconsin, Madison, Wis. 1430 Chandler St.

Brunk, H. D. Fellow, Rice Inst., Houston, Tex.

Bruton, G. S. Asso. Prof., Univ. of the South, Sewanee, Tenn.

Bryan, N. R. Asso. Prof., Univ. of Maine, Orono, Me. 4 University P1.

Bubb, C. R. Univ. of Oregon, Eugene, Ore.

Buchanan, Daniel. Dean, Faculty of Arts and Science, Univ. of British Columbia, Vancouver, B.C., Canada.

Buchanan, H. E. Prof., Tulane Univ., New Orleans, La.

Buck, Thomas. Prof., Univ. of California, Berkeley, Calif. Faculty Club.

Buell, Dr. E. L. Instr., Northwestern Univ., Evanston, I11.

Bula, Clotilde A. Asst. Prof., Univ. of Buenos Aires, Buenos Aires, Argentina; Research Worker, Univ. of Rosario, Rosario, Argentina. Calle Moreno 364, Rosario, Argentina.

Bull, Adelaide W. Research Representative, Encyclopoedia Britannica, Inc., 20 Wacker Dr., Chicago, I1l. 47 Montclair Ave., Montclair, N.J.

Bullard, J. A. Prof., Coll. of Engineering, Univ. of Vermont and State Agric. Coll., Burlington, Vt. 110 Summit St.

Bullitt, W. M. Lawyer, 1711 Kentucky Home Life Bldg., Louisville, Ky.

Bulloch, G. E. Asst. Engineer, New Jersey Bell Telephone Co., 540 Broad St., Newark, N.J. 290 Livingston Ave., Lyndhurst, N.J.

Bullock, R. C. Asso. Prof., North Carolina State Coll., Raleigh, N.C. 5548 State College Station.

Bumer, C. T. Prof., Kenyon Coll., Gambier, Ohio.

Bunyan, L. H. Asst. Prof., Rutgers Univ., New Brunswick, N.J.

Burcham, Dr. P. B. Instr., Central Coll., Fayette, Mo. 510 N. Church St.

Bures, Dr. C. E. National Jewish Hospital, Denver, Colo.

Burgess, Louis. Attorney, 233 Broadway, New York, N.Y.

Burgess, Dr. R. W. Chief Economist, Western Electric Co., 195 Broadway, New York, N.Y. 
Burington, R. S. Asso. Prof., Case School of Applied Science, Cleveland, Ohio. Research Mathematician, Bureau of Ordnance, U. S. Navy Dept., Washington, D.C. 5200 Carlin Springs Road, Arlington Forest, Va.

Burk, J. D. Asst. Prof., Univ. of Toronto, Toronto, Ont., Canada.

§Burke, Barbara H. (Mrs. T. F.). 581 San Antonio St., San Diego, Calif.

Burke, Sister Leonardo. Prof., Regis Coll., Weston, Mass.

Burkett, F. J. H. Asst. Prof., Union Coll., Schenectady, N.Y. 1030 Park Ave.

Burks, Dr. A. W. Instr., Moore School of Electrical Engineering, Univ. of Pennsylvania, Philadelphia, Pa.

Burnam, J. E. Prof., Hardin-Simmons Univ., Abilene, Tex. 1141 Grape St.

Burns, H. E. Asst. Prof., Indiana Univ. Extension, East Chicago, Ind. 3901 Indianapolis Blvd.

Burns, Sister M. Roberdette. Instr., Clarke Coll., Dubuque, Iowa.

Burr, I. W. Asst. Prof., Purdue Univ., Lafayette, Ind.

Burt, Mary M. (Mrs. L. N.). Retired. 800 24th Ave. N., St. Petersburg, Fla.

Burton, E. T. Research Engineer, Bell Telephone Labs, 463 West St., New York, N.Y.

$\S$ Burton, L. P. No address.

Burwell, Dr. W. R. 3311 Perkins Ave., Cleveland, Ohio.

Busemann, Dr. Herbert. Instr., Illinois Inst. of Tech., Chicago, Ill.

Bush, K. A. 7 Walling Blvd., Oneonta, N.Y.

Bush, L. E. Prof., Coll. of St. Thomas, St. Paul, Minn.

Bush, Vannevar. Pres., Carnegie Inst., Washington, D.C. 1530 P St. N.W. Dir., Office of Scientific Research and Development.

Bushey, Hobart. Asso. Prof., Hunter Coll., 695 Park Ave., New York, N.Y.

Bushey, Jewell H. (Mrs. Hobart). Asso. Prof., Hunter Coll., 695 Park Ave., New York, N.Y.

Bushkovitch, A. V. Asso. Prof. of Physics and Philosophy, Coll. of Charleston, Charleston, S.C.

Bussey, W. H. Asst. Dean, Coll. of Science, Literature and the Arts, and Prof., Univ. of Minnesota, Minneapolis, Minn. 106 Folwell Hall.

Butler, J. F. Univ. of Detroit, Detroit, Mich.

Butler, L. G. Instr., State Coll. of Washington, Pullman, Wash.

Butter, F. A. Asst. Prof., Univ. of Southern California, Los Angeles, Calif. Box 24.

§Byerly, R. W. 225 Broadway, New York, N.Y.

Byrne, W. E. Prof., Virginia Military Inst., Lexington, Va. Box 836. Col., Virginia Militia.

Cahn, Albert. Research Asst., Inst. of Meteorology, Univ. of Chicago, Chicago, Ill.

Cairns, S. S. Asst. Prof., Queens Co11., Flushing, N.Y. 42-14 149th P1.

Cairns, W. D. Prof. Emeritus, Oberlin Coll., Oberlin, Ohio. 153 E. College St.

Calkin, J. w. Asst. Prof., Illinois Inst. of Tech., Chicago, I1l. Bureau of Ordnance U. S. Navy Dept., Washington, D.C. 7813 Old Chester Road, Bethesda, Md.

Calkins, Helen. Prof., Pennsylvania Coll. for Women, Pittsburgh, Pa.

Callahan, Ethel B. Asso. Prof., Shepherd State Teachers Coll., Shepherdstown, W.Va.

Callaway, Iris. Asso. Prof., Univ. of Georgia, Athens, Ga. 258 Hill St.

Cameron, E. A. Asso. Prof., Univ. of North Carolina, Chapel Hill, N.C. 121 Kenan St. Ground School Instr., U.S.N. Pre-Flight School, Chapel Hill, N.C.

Cameron, R. H. Asst. Prof., Massachusetts Inst. of Tech., Cambridge, Mass. 17 Frost Road, Belmont, Mass.

†Camp, B. H. Prof., Wesleyan Univ., Middletown, Conn. 110 Mt. Vernon St.

Camp, C. C. Prof., Univ. of Nebraska, Lincoln, Neb.

Camp, E. J. Asso. Prof., Macalester Coll., St. Paul, Minn.

Campaigne, Dr. H. H. Instr., Univ. of Minnesota, Minneapolis, Minn. Lt. (j.g.), U.S.N.R., U. S. Navy Dept., Washington, D.C. 232 N. Fillmore St., Arlington, $V a$.

Campbell, A. D. Prof., Syracuse Univ., Syracuse, N.Y. 208 Westminster Ave. 
Campbell, Dr. D. F. Consulting Actuary. 160 N. LaSalle St., Chicago, Ill.

Campbell, Dr. G. A. Retired. 129 Bellevue Ave., Upper Montclair, N.J.

Campbell, J. W. Prof., Univ. of Alberta, Edmonton, Alta., Canada.

Campbell, W. B. Asso. Prof. of Engineering, Pennsylvania Military Coll., Chester, Pa. 6848 N. 7th St., Philadelphia, Pa.

Candy, A. L. Prof. Emeritus, Univ. of Nebraska, Lincoln, Neb. Station A.

†Canning, Joseph. Roundhouse Foreman, Northern Pacific Railway Co., Garrison, Mont.

Cannon, Dr. E. W. Instr., Univ. of Delaware, Newark, Del. Cannon, Del.

Capecelatro, Dr. Achille. 100 E. Mosholu Pkwy. S., Bronx, New York, N.Y.

Caraher, Loula M. Asso. Prof., Arkansas State Teachers Coll., Conway, Ark. 342 Donaghey Ave.

Carey, C. E. Prof., State Teachers Coll., Clarion, Pa. 125 S. 5th Ave.

Carey, E. F. A. Asso. Prof., Montana State Univ., Missoula, Mont.

Caris, P. A. Asso. Prof., Univ. of Pennsylvania, Philadelphia, Pa. 717 Shadeland Ave., Drexel Hill, $\mathrm{Pa}$.

Carlen, Mildred E. Registrar, Graduate School, and Instr., Brown Univ., Providence, R.I.

Carlitz, Leonard. Asso. Prof., Duke Univ., Durham, N.C. 4674 Duke Station.

$\S$ Carlson, B. E. Asst., Yale Univ., New Haven, Conn. 109 Davenport Ave.

Carlson, Elizabeth. Ässt. Prof., Univ. of Minnesota, Minneapolis, Minn. 3024 14th Ave. S.

Carman, M. G. Prof., Murray State Teachers Coll., Murray, Ky.

Carmichael, R. D. Dean, Graduate School, Univ. of Illinois, Urbana, I11. 207 W. Washington Blvd.

Carner, F. E. Stress Analyst, Airplane Div., Curtiss-Wright Corp., Buffalo, N.Y. 303 Humboldt Pkwy.

Carpenter, A. F. Prof., Univ. of Washington, Seattle, Wash.

Carpenter, R. V. A. Retired. 66 Park Ave., New York, N.Y.

Carroll, C. L. Instr., Georgia School of Tech., Atlanta, Ga.

Carroll, I. S. Asso. Prof., Syracuse Univ., Syracuse, N.Y. 511 Comstock Ave.

Carroll-Rusk, Evelyn (Mrs. W. S. Rusk). Dean and Prof., Wells Coll., Aurora, N.Y.

$\S$ Carruth, Dr. P. W. 2843 Southington Road, Shaker Heights, Cleveland, Ohio. $2 d$ Lt., U.S.A., Fort Bliss, Tex.

†Carruth, w. M. Prof., Hamilton Coll., Clinton, N.Y. College Hill.

Carslaw, H. S. Prof. Emeritus, Univ. of Sydney, Sydney, Australia. Burradoo, Australia.

Carson, Dr. A. B. Instr., Louisiana State Univ., University, La. Care of Mrs. Jessie Roth, 748 N. Laramie Ave., Chicago, I1l. $2 d$ Lt., A.U.S., Miami Beach, Fila.

Cartan, E. J. Prof., Univ. of Paris, Paris, France. 95 Blvd. Jourdan, Paris, 14, France.

Carter, E. R. Actuary, Washington National Ins. Co., Evanston, I11.

Carter, H. C. Prof., Mary Washington Coll., Fredericksburg, Va. 1307 Littlepage St.

Carter, P. S. Research Engineer, Radio Corp. of America Labs., Rocky Point, N.Y.

§Carter, w. C. 5737 University Ave., Chicago, I11. Ensign, U.S.N.R.

Carver, W. B. Prof., Cornell Univ., Ithaca, N.Y. White Hall.

Case, J. E. Prof., St. Louis Univ., St. Louis, Mo.

Cassidy, Dr. W. F. St. John's Univ., Brooklyn, N.Y.

Cassity, Dr. C. R. Instr., Univ. of Alabama, University, Ala. Office of the Chief Signal Officer, U.S. War Dept., Washington, D.C. 1110 N. Kensington St., Arlington, $V a$.

Castle, S. N. Engineer, Kalanianaole Highway, Honolulu, Hawaii.

Caton, W. B. Asso. Prof., Southwestern Coll., Winfield, Kan.

Caywood, T. E. Fellow, Harvard Univ., Cambridge, Mass. 1734 Cambridge St. Research Asso., Cruft Lab., Harvard Univ.

Cederberg, W. E. Prof., Augustana Coll., Rock Island, I11. 2542 22ז $1 / 2$ Ave.

Cell, J. W. Asso. Prof., North Carolina State Coll., Raleigh, N.C. 
de Cesare, E. A. Prof., Univ. of LaPlata, LaPlata, Argentina. Calle Rivadavia 1757, Buenos Aires, Argentina.

§Chandrasekhar, Subrahmanyan. Asso. Prof., Yerkes Observ., Univ. of Chicago, Williams Bay, Wis.

Chaney, J. G. Asst. Prof., Agric. and Mech. Coll. of Texas, College Station, Tex. Lt. (j.g.), U.S.N.R.

Chanler, Dr. Josephine H. Asso., Univ. of Illinois, Urbana, I1l. 364 Math. Bldg. Chapman, D. G. Meteorologist, Meteorological Services of Canada. 25 Arlington Ave., Westmount, Quebec, Canada.

Charnes, Abraham. Harvard Univ., Cambridge, Mass. 4 Arrow St.

Charney, J. G. Asst., Univ. of California at Los Angeles, Los Angeles, Calif.

Chatland, Harold. Asst. Prof., Montana State Univ., Missoula, Mont. 518 Evans Ave.

Cheney, W. F. Prof., Univ. of Connecticut, Storrs, Conn.

Cherry, T. M. Prof., Melbourne Univ., Melbourne, Australia.

Chesna, John. Physicist, Bausch and Lomb Optical Co., Rochester, N.Y. 29 Barons St.

Chevalley, Claude. Asst. Prof., Princeton Univ., Princeton, N.J. Fine Hall.

Chittenden, E. W. Prof., State Univ. of Iowa, Iowa City, Iowa. 221 Physics Bldg.

Christie, Dr. D. E. Instr., Bowdoin Coll., Brunswick, Me. Searles Physics Lab.

Church, Alonzo. Asso. Prof., Princeton Univ., Princeton, N.J. Fine Hall.

Church, Randolph. Asst. Prof., Postgraduate School, U.S. Naval Acad., Annapolis, Md. 316 N. Glen Ave. Lt., U.S.N.R., Office of Chief of Naval Operations, U.S. Navy Dept., Washington, D.C.

Churchill, R. V. Prof., Univ. of Michigan, Ann Arbor, Mich. 924 S. Forest Ave.

§Civin, Dr. Paul. Instr., Univ. of Michigan, Ann Arbor, Mich.

Clark, C. E. Asst. Prof., Purdue Univ., Lafayette, Ind.

$\S$ Clark, C. L. Univ. of Virginia, University Station, Charlottesville, Va. Monroe Apts., Brandon Ave.

Clarke, E. H. Prof., Hiram Coll., Hiram, Ohio.

Clarkson, Helen E. Prof., Jacksonville Coll., Jacksonville, Tex. 1806 Emmet St., Omaha, Neb.

Clarkson, J. A. Asst. Prof., Univ. of Pennsylvania, Philadelphia, Pa. College Hall.

Clarkson, J. M. Asso. Prof., North Carolina State Coll., Raleigh, N.C. State Co1lege Station.

Claytor, Dr. W. W. S. 1710 P St. N.W., Washington, D.C.

Clement, Dr. Mary Dean. Instr., Wells Coll., Aurora, N.Y.

§Clement, P. A. Asst., Univ. of California at Los Angeles, Los Angeles, Calif. 220 Diamond, Laguna Beach, Calif.

Clements, G. R. Prof., U.S. Naval Acad., Annapolis, Md. 7 Thompson St.

Clifford, A. H. Asst. Prof., Massachusetts Inst. of Tech., Cambridge, Mass. Lt. (j.g.), U.S.N.R., 3130 Wisconsin Ave. N.W., Washington, D.C.

$\S$ Clifford, H. E. Dean, Engineering School, Harvard Univ., Cambridge, Mass. Pierce Hall.

Clippinger, Dr. R. F. Instr., Carnegie Inst. of Tech., Pittsburgh, Pa. 235 Lehigh St.

Coble, A. B. Prof., Univ. of Illinois, Urbana, I11. 702 W. Washington Blvd.

Coburn, Dr. Nathaniel. Instr., Univ. of Texas, Austin, Tex. 5401 Aurora Dr.

Coddington, E. A. Junior Physicist, Ordnance Lab., Navy Yard, U.S. Navy Dept., Washington, D.C. 808 Portland St. S.E.

Coe, C. J. Asst. Prof., Univ. of Michigan, Ann Arbor, Mich.

Coffin, L. M. Prof., Coe Coll., Cedar Rapids, Iowa. 1027 2d Ave.

Cohen, Abraham. Prof. Emeritus, Johns Hopkins Univ., Baltimore, Md.

$\S$ Cohen, Dr. I. S. Asst., Inst. for Advanced Study, Princeton, N.J.

Cohen, Leo. 1337 Tuckerman St. N.W., Washington, D.C.

Cohen, L. w. Prof., Univ. of Kentucky, Lexington, Ky.

Cohen, Teresa. Asso. Prof., Pennsylvania State Coll., State College, Pa. 315 S. Atherton St.

§Cohn, Harvey. Asst., New York Univ., New York, N.Y. 847 E. 172d St., Bronx.

$\S$ Cohn, R. M. 905 West End Ave., New York, N.Y. Inspector of Radio Equipment, U.S.A., Signal Corps. 5000 Wissahukon Ave., Philadelphia, Pa. 
Coker, E. C. Asst. Prof., Clemson Coll., Clemson, S.C. Box 284.

$\S$ Cole, K. S. Asso. Prof., Columbia Univ., New York, N.Y. Inst. for Advanced Study, Princeton, N.J.

Cole, Dr. Nancy. Instr., Sweet Briar Coll., Sweet Briar, Va.

Cole, Dr. R. H. Instr., Univ., of Western Ontario, London, Ont., Canada.

Coleman, A. J. Sec., Student Christian Movement, Univ. of Toronto, Toronto, Ont., Canada. Hart House.

Coleman, Dr. J. B. 620 Bull St., Columbia, S.C.

Coleman, W. B. Instr., Agric. and Mech. Coll. of Texas, College Station, Tex. 253 Faculty Exch. 2d Lt., U.S.A., Miami Beach, Fla.

Coler, Dr. M. A. Tech. Dir., Engineering Products Div., Paragon Paint and Varnish Corp., Long Island City, N.Y. 328 W. 86th St., New York, N.Y.

Collier, Myrtie. Prof., Immaculate Heart Coll., Los Angeles, Calif. 1307 S. Beverly Glen Blvd.

Collingwood, Dr. E. F. Trinity Coll., Univ. of Cambridge, Cambridge, England. Lilburn Tower, Alnwick, Northumberland, England.

$†$ Collins, O. C. Asst. Prof. of Astronomy, Univ. of Nebraska, Lincoln, Neb. The Observatory.

Colvin, B. H. Asst., Univ. of Wisconsin, Madison, Wis.

Comegys, Dr. Esther. Instr., Univ. of Maine, Orono, Me. 6 University P1.

Comenetz, Dr. George. Tutor, St. John's Coll., Annapolis, Md.

§Comer, Louise M. Asst., Barnard Coll., Columbia Univ., New York, N.Y. Milbank Hall.

Comfort, Dr. E. G. H. Instr., Univ. of Arkansas, Fayetteville, Ark. Capt., U.S.A., Kelly Field, Tex.

Comstock, C. E. Prof. Emeritus, Bradley Polytech. Inst., Peoria, Ill. 203 Fredonia Ave.

Conkwright, N. B. Asst. Prof., State Univ. of Iowa, Iowa City, Iowa. 209-B Physics Bldg.

Constable, Mary L. Philadelphia High School for Girls, Philadelphia, Pa. The Whittier, 140 N. 15th St.

Conwell, Dr. G. M. St. Paul's School, Concord, N.H. 307 Pleasant St.

Conwell, H. H. Dean and Prof., Beloit Coll., Beloit, Wis. 1621 Emerson St.

Cook, A. J. Asso. Prof., Univ. of Alberta, Edmonton, Alta., Canada.

Cook, R. H. Asst. Prof., South Dakota State School of Mines, Rapid City, S.Dak.

Cook, Sister Rose M. Prof., Loretto Heights Coll., Loretto, Colo.

Cooley, H. R. Asst. Prof., Washington Square Coll., New York Univ., New York, N.Y.

†Coolidge, J. L. Prof. Emeritus, Harvard Univ., Cambridge, Mass. 27 Fayerweather St.

§Coolidge, T. J. Vice Pres., The First National Bank of Boston, 67 Milk St., Boston, Mass.

Coombs, W. C. Radio Div., Bureau of Ships, U.S. Navy Dept., Washington, D.C. 1833 Ingelside Ter. N.W.

Cooper, Clara M. St. Vincent's Coll., Shreveport, La.

Cooper, Dr. Elizabeth M. Hunter Coll. High School, New York, N.Y. 201 E. 71 st St.

Cope, T. F. Asso. Prof., Queens Coll., Flushing, N.Y. 33-69 167th St.

Copeland, A. H. Asso. Prof., Univ. of Michigan, Ann Arbor, Mich. 616 Oswego St.

†Copeland, Lennie P. Prof., Wellesley Coll., Wellesley, Mass.

Copenhagen, Helen M. Univ. of Washington, Seattle, Wash.

Copson, E. T. Prof., University Coll., St. Andrews Univ., Dundee, Scotland.

Coral, Max. Asst. Prof., Wayne Univ., Detroit, Mich.

Cordrey, W. A. Prof., Southeastern Louisiana Coll., Hammond, La. 3930 Clermont Dr., New Orleans, La.

Corliss, J. J. Prof., De Paul Univ., Chicago, Ill.

Corona, Sister Maria. Dean, Coll. of Mt. St. Joseph, Mt. St. Joseph, Ohio.

Corral-Aleman, J. I. Dir., Mountains and Mines of the Republic of Cuba, Havana, Cuba. Calzada esquina 13, Vedado.

Cosby, Dr. Byron. Instr., U. S. Naval Acad., Annapolis, Md. 
Cothran, Dr. J. C. Chemistry Dept., State Teachers Coll., Duluth, Minn. 512 N. 19th Ave. E.

Courant, Richard. Prof., Washington Square Coll., New York Univ., New York, N.Y. 142 Calton Road, New Rochelle, N.Y.

Court, N. A. Prof., Univ of Oklahoma, Norman, Okla. 425 W. Eufaula St.

Cowan, G. P. St. John's Univ., Brooklyn, N.Y.

Cowgill, A. P. Asst. Prof., Syracuse Univ., Syracuse, N.Y. 519 Allen St. Maj., U.S.A., retired.

Cowles, W. H. H. Acting Dir., School of Science and Tech., Pratt Inst., Brooklyn, N.Y. 132 Joralemon St.

Cox, Dr. L. C. English Hotel, Indianapolis, Ind.

Cox, Mary J. Univ, of Virginia, University Station, Charlottesville, Va. 21 University Circle.

Coxeter, H. S. M. Asst. Prof., Univ. of Toronto, Toronto, Ont., Canada. 24 Strathearn, Blvd.

Craig, A. T. Asso. Prof., State Univ. of Iowa, Iowa City, Iowa. 119 Physics Bldg.

Craig, C. C. Prof., Univ. of Michigan, Ann Arbor, Mich. 3020 Angell Hall.

Craig, H. v. Prof., Univ. of Texas, Austin, Tex. 1816 W. 36th St.

Cramblet, W. H. Pres., Bethany Coll., Bethany, W.Va.

Cramer, G. F. Asst. Prof., Tulane Univ., New Orleans, La. 1333 Lowerline St. Lt., U.S.N.R. 3026 Porter St. N.W., Washington, D.C.

Cramer, R. H. Aerodynamics Section, Curtiss-Wright Corp., Buffalo, N.Y. 500 Grand Ave., Rochester, N.Y.

Cramlet, C. M. Asso. Prof., Univ. of Washington, Seattle, Wash.

§Crane, C. H. Pres., St. Joseph Lead Co., 250 Park Ave., New York, N.Y.

Crathorne, A. R. Prof., Univ. of Illinois, Urbana, Ill. 802 Pennsylvania Ave.

Craw, A. R. Instr., De Paul Univ., Chicago, I11. 5601 Drexel Ave. Ensign, U.S.N.R.

Crawford, Jane E. Editorial Asst., American Mathematical Society, 531 W. 116th St., New York, N.Y.

$\S$ Crawford, W. S. H. Asst., Univ. of Minnesota, Minneapolis, Minn. 103 Folwell Hall.

Creane, Sister James S. Instr., Sacred Heart High School, Indianapolis, Ind. 10 E. Palmer St.

Cromwell, J. W. 1815 13th St., Washington, D.C.

Cronvich, Dr. L. L. Asst. Stress Analyst, McDonnell Aircraft Corp., St. Louis, Mo. 7023 Stanford Ave.

Crosby, D. R. Box 543, Banff, Alta., Canada. Flying Officer, R.C.A.F., No. 9 Air Observors School, St. Johns, Quebec, Canada.

Crout, P. D. Asso. Prof., Massachusetts Inst. of Tech., Cambridge, Mass.

Crow, Dr. E. L. Bureau of Ordnance, U.S. Navy Dept., Washington, D.C. 119 5th St., N.E.

Culver, M. M. Prof., Univ. of Pittsburgh, Pittsburgh, Pa.

Cummings, H. K. Chief, Automotive Power Plants Section, National Bureau of Standards, U. S. Dept. of Commerce, Washington, D.C. 4213 Ellicott St. N.W.

§Cunningham, Dr. A. B. Instr., DuBois Undergraduate Center, Pennsylvania State College, Dubois, Pa.

§Curran, Mary T. Johns Hopkins Univ., Baltimore, Md. 5 E. Preston St.

Currier, A. E. Asst. Prof., U. S. Naval Acad., Annapolis, Md.

Curry, H. B. Prof., Pennsylvania State Coll., State College, Pa. Asso. Mathematician, Frankford Arsenal, Philadelphia, Pa. 6709 N. 6th St.

Curtis, H. B. Prof., Lake Forest Coll., Lake Forest, I11. 11 College Campus.

Curtiss, D. R. Prof., Northwestern Univ., Evanston, I11.

Curtiss, J. H. Asst. Prof., Cornell Univ., Ithaca, N.Y. White Hall.

Cutler, E. H. Asst. Prof., Lehigh Univ., Bethlehem, Pa.

Dadourian, H. M. Prof., Trinity Coll., Hartford, Conn. 125 Vernon St.

Dalaker, H. H. Prof. Emeritus, Univ. of Minnesota, Minneapolis, Minn. 523 Walnut St. S.E.

$\dagger$ Dalal, R. D. Care of Swiss Bank Corp., 11c Regent St., London, S.W. 1, England.

Daly, Dr. J. F. Instr., Catholic Univ. of America, Washington, D.C. 
Danielson, J. O. 2319 Maryland Ave., Superior, Wis.

Dantzig, G. B. Asso. Statistician, U. S. War Dept., Washington, D.C. 4720 7th Road N., Arlington, Va.

Dantzig, Tobias. Prof., Univ. of Maryland, College Park, Md. University Lane.

Darkow, Marguerite D. Asso. Prof., Hunter Coll., 695 Park Ave., New York, N.Y. 16 E. 82nd St.

Daugherty, R. D. Asst. Prof., Kansas State Coll. of Agric. and Applied Science, Manhattan, Kan. Box 64.

Daum, Dr. J. A. Instr., Agric. and Mech. Coll. of Texas, College Station, Tex. 3804 Ames Ave., Omaha, Neb. $2 d$ Lt., U.S.A., Camp Bowie, Tex.

Daus, P. H. Asso. Prof., Univ. of California at Los Angeles, Los Angeles, Calif.

Davids, Dr. Norman. Junior Physicist, U. S. Engineer Div., Cincinnati Testing Lab., Mariemont, Ohio.

Davidson, Anthony. Prof., Wilberforce Univ., Wilberforce, Ohio. 316 E. Church St., Xenia, Ohio.

Davis, D. R. Prof., New Jersey State Teachers Coll., Upper Montclair, N.J.

Davis, H. A. Asso. Prof., West Virginia Univ., Morgantown, W.Va. 307 Duquesne Ave.

$\dagger$ Davis, H. N. Pres., Stevens Inst. of Tech., Hoboken, N.J.

Davis, James Edgar. Asso. Prof., Central Y.M.C.A. Coll., 19 S. LaSalle St., Chicago, Ill.

Davis, James Elmer. Prof., Drexel Inst. of Tech., Philadelphia, Pa. Colonel, U.S.A.

§Davis, M. E. Asso. Actuary, Metropolitan Life Ins. Co., 1 Madison Ave., New York, N.Y.

Davis, W. M. Asst. Prof., Cornell Coll., Mt. Vernon, Iowa. 603 N. 6th St. W.

Davison, Charlotte I. Prof., Wilson Coll., Chambersburg, Pa.

Dawkins, W. S. 3310 Carlisle Ave., Baltimore, Md.

$\S$ Day, A. S. Asst., Yale Univ., New Haven, Conn. 2723 Yale Station.

Day, Dr. M. M. Instr., Univ. of Illinois, Urbana, Ill. 312 E. Healey St., Champaign, Ill.

Dean, Alice C. Fellow and Acting Lib., Rice Inst., Houston, Tex.

Dean, J. P. Radio Engineer, U.S.A. Signal Corps, Hq. Bldg. 100, Presidio of San Francisco, Calif. 1635 Gough St.

Dearborn, D. C. Asso. Prof. and Registrar, Catawba Coll., Salisbury, N.C.

Dearman, C. C. Instr., East Central Junior Coll., Decatur, Miss. Ground School Instr., U. S. Naval Air Station, Pensacola, Fla. 7 S. H St.

Decherd, Mary E. Asst. Prof., Univ. of Texas, Austin, Tex. 2313 Nueces St.

DeCicco, Dr. John. Instr., Illinois Inst. of Tech., Chicago, I1l. 6340 Blackstone Ave.

Deck, L. J. Prof., Muhlenberg Coll., Allentown, Pa. 232 N. 15th St.

Decker, F. F. Prof., Syracuse Univ., Syracuse, N.Y. 312 Marshall St.

DeCleene, Rev. L. A. V. Prof., St. Norbert's Abbey, West De Pere, Wis.

DeCou, E. E. Prof. Emeritus, Univ. of Oregon, Eugene, Ore. 929 Hilyard St.

$\dagger$ Dederick, Dr. L. S. Prin. Mathematician, Ballistic Research Lab., Aberdeen Proving Ground, Md.

DeFoe, O. K. Prof., St. Louis Coll. of Pharmacy, St. Louis, Mo. 4588 Parkview P1.

Dehn, Dr. M. W. Visiting Lecturer, Illinois Inst. of Tech., Chicago, I11.

Deimel, R. F. Prof., Stevens Inst. of Tech., Hoboken, N.J.

$\nmid$ DeLong, I. M. Prof. Emeritus, Univ. of Colorado, Boulder, Colo. 2009 13th St.

DeLury, Dr. D. B. Lecturer, Univ. of Toronto, Toronto, Ont., Canada.

Demers, M. R. 1st Lt., U.S.A., Air Forces, Gravelly Point, Washington, D.C.

Denbow, Carl. Asst. Prof., Ohio Univ., Athens, Ohio. Valley View Dr.

Dennis, J. J. Prof., Clark Univ., Atlanta, Ga. 1315 Marcy St.

Denton, W. W. Dean, Great Lakes Coll., 4211 3d Ave., Detroit, Mich.

DePrima, C. R. Asst., New York Univ., New York, N.Y. 307 6th Ave., Paterson, N.J.

Derby, G. T. Maj., U.S.A., Corps of Engineers, New Orleans, La. 1403 Calhoun St.

Derry, Douglas. Asst. Prof., Univ. of Saskatchewan, Saskatoon, Sask., Canada. 619 Temperance St. 
§Derthick, P. W. Asst., Univ. of Kentucky, Lexington, Ky. Signal Corps, U.S. War Dept., Washington, D.C. 5 Columbia Ave., Takoma Park, D.C.

Diamond, A. H. Prof., Oklahoma Agric. and Mech. Coll., Stillwater, Okla. $2 d$ Lt., U.S.A., Air Forces, Monroe Tower Hotel, Miami Beach, Fla.

Dickson, L. E. Prof. Emeritus, Univ. of Chicago, Chicago, I11.

Dieckmann, H. C. Asst. Prof., Occidental Coll., Los Angeles, Calif.

Dietzold, R. L. Research Engineer, Bell Telephone Labs., 463 West St., New York, N.Y. 34 W. 11th St.

Dilworth, Dr. R. P. Instr., Yale Univ., New Haven, Conn. North Sheffield Hall.

Dimick, C. E. Prof., U.S. Coast Guard Acad., New London, Conn. Com., U.S. C.G.

Dimsdale, Dr. Bernard. Instr., Univ. of Idaho, Moscow, Idaho. Hastings, Neb.

Dines, L. L. Prof., Carnegie Inst. of Tech., Pittsburgh, $\mathrm{Pa}$.

Disert, Margaret C. Dean, Wilson Coll., Chambersburg, Pa. Lt., U.S.N.R., Widener Bldg., Philadelphia, $\mathrm{Pa}$.

Dix, Dr. C. H. Chief Seismologist, United Geophysical Co. 628 Alameda St., Altadena, Calif.

Dixon, A. L. Prof., Magdalen Coll., Univ. of Oxford, Oxford, England.

Dixon, W. J. Instr., Univ. of Oklahoma, Norman, Okla.

Dobbie, Dr. J. M. Instr., Northwestern Univ., Evanston, I11.

DoBell, H. A. Prof., New York State Coll. for Teachers, Albany, N.Y.

Dodd, E. L. Prof., Univ. of Texas, Austin, Tex. 3012 West Ave.

Doob, J. L. Asst. Prof., Univ. of Illinois, Urbana, Ill. 4431 Greenwich Pkwy. N.W., Washington, D.C.

Doole, H. P. Asst. Prof., Univ. of Nebraska, Lincoln, Neb.

Dorroh, J. L. Asst. Prof., Louisiana State Univ., University, La.

Dorwart, H. L. Asst. Prof., Washington and Jefferson Coll., Washington, Pa. Gabby Heights, R.F.D. 6.

Dostal, B. F. Asst. Prof., Univ. of Florida, Gainesville, Fla. Peabody Hall.

Dotterer, R. H. Prof., Philosophy Dept., Pennsylvania State Coll., State College, Pa. 825 W. Foster Ave.

Douglas, Jesse. Prof., Columbia Univ., New York, N.Y.

Dowker, Dr. C. H. Instr., Johns Hopkins Univ., Baltimore, Md.

Downey, J. F. Consulting Engineer, American Associated Consultants Inc., 250 Park Ave., New York, N.Y. 20 N. Broadway, White Plains, N.Y.

Downing, H. H. Prof., Univ. of Kentucky, Lexington, Ky. 138 State St.

Downs, Dr. T. L. Instr., Trinity Coll., Hartford, Conn. Lt. (j.g.) U.S.N.R., U.S. Naval Acad., Annapolis, Md.

$\S$ Doyle, Martina E. Connecticut General Life Ins. Co., Hartford, Conn. 316 White St.

Doyle, Dr. T. C. Instr., Stanford Univ., Stanford University, Calif.

Doyle, W. C. Asst. Prof., Rockhurst Coll., 5225 Troost Ave., Kansas City, Mo.

Dresch, Dr. F. W. Instr., Univ. of California, Berkeley, Calif. Lt. (j.g.), U.S.N.R., U.S. Naval Proving Ground, Dahlgren, Va.

Dresden, Arnold. Prof., Swarthmore Coll., Swarthmore, Pa. 606 Elm Ave.

Dressel, F. G. Asst. Prof., Duke Univ., Durham, N.C. 309 Francis St.

Dressler, R. F. Univ. of Pennsylvania, Philadelphia, Pa. 115 S. 42d St. Electrical Engineer, Radia Corp. of America, Camden, N.J.

Dribin, Dr. D. M. Junior Research Analyst, Office of Chief Signal Officer, U. S. War Dept., Washington, D.C.

Drum, M. L. Prof. of Surveying, Bucknell Univ., Lewisburg, Pa. 55 S. Water St.

§Dubisch, Roy. Instr., Montana State Univ., Missoula, Mont.

Duffin, Dr. R. J. Physicist, Terrestrial Magnetism Dept., 5241 Broad Branch Road N.W., Washington, D.C.

Duncan, Dr. D. C. Instr., Los Angeles City Coll., Los Angeles, Calif.

Dunford, Margaret J. Southeastern High School, Detroit, Mich. 2526 Glendale Ave.

Dunford, Nelson. Asst. Prof., Yale Univ., New Haven, Conn. 215 Norton St.

Dunkel, Otto. Asso. Prof. Emeritus, Washington Univ., St. Louis, Mo.

Durand, Janet C. Prof., Beaver Coll., Jenkintown, Pa. 144 Walnut St.

†Durand, W. F. Prof. Emeritus, Stanford Univ., Stanford University, Calif. 540 Aero Lab. 
Durell, Dr. Fletcher. Belleplain, N.J.

Duren, W. L. Asso. Prof., Tulane Univ., New Orleans, La.

Durfee, Walter H. Dean, Hobart Coll., Geneva, N.Y. Coxe Hall.

§Durfee, William H. Instr., Cornell Univ., Ithaca, N.Y.

Dushnik, Ben. Asst. Prof., Univ. of Michigan, Ann Arbor, Mich. 205 W. Engi. neering Bldg.

Duthie, Dr. W. D. Instr., Univ. of Michigan, Ann Arbor, Mich. Lt., U.S.N.R., Postgraduate School, U.S. Naval Acad., Annapolis, Md.

§Dutka, Jacques. 56 W. 180th St., Bronx, New York, N.Y.

Dwyer, P. S. Asst. Prof., Univ. of Michigan, Ann Arbor, Mich. 2509 James St.

Dye, L. A. Asst. Prof., The Citadel, Charleston, S.C.

Dyer-Bennet, Dr. John. Instr., Vanderbilt Univ., Nashville, Tenn. U.S.A., Camp Blanding, Fla.

Dysart, Robert. Certified Public Accountant, 50 State St., Boston, Mass.

Eachus, Dr. J. J. Instr., Purdue Univ., Lafayette, Ind. Lt. (j.g.), U.S.N.

Eagle, E. L. 1320 Huffman Ave., Dayton, Ohio. Supervisor of Training, U.S.A., Air Corps, Patterson Field, Air Depot, Fairfield, Ohio.

Earl, J. M. Prof., Municipal Univ. of Omaha, Omaha, Neb. 528 S. 53d St.

Eaton, Dr. J. E. Instr., Queens Coll., Flushing, N.Y. 43-55 Kissena Blvd.

Eaves, E. D. Asso. Prof., Univ. of Tennessee, Knoxville, Tenn. University Station.

Eberhart, Paul. Asso. Prof., Washburn Municipal Univ. of Topeka, Topeka, Kan.

Eberlein, W. F. Propeller Research Analyst, U. S. Navy Dept., Washington, D.C. $2223 \mathrm{H}$ St. N.W.

Eberman, J. W. 141-72 85th Road, Jamaica, N.Y.

Echols, R. L. Asst. Prof., Postgraduate School, U. S. Naval Acad., Annapolis, Md.

Eckweiler, H. J. Instr., New York Univ., New York, N.Y. 9303 49th Ave., Elmhurst, N.Y.

§Eddy, R. P. Rockton, I11. U.S.A., Signal Corps, Burr School, 1621 Wabansia, Chicago, Ill.

Edington, W. E. Prof., DePauw Univ., Greencastle, Ind. E. Franklin St.

Edison, T. M. Pres., Calibron Products Inc., 51 Lakeside Ave., West Orange, N.J.

Efird, Laura C. Hugh Morson High School, Raleigh, N.C. 506 Cutler St.

Eide, Margaret C. (Mrs. R. B.). Asso. Prof., State Teachers Coll., River Falls, Wis. 308 S. 2d St.

Eiesland, J. A. Prof. Emeritus, West Virginia Univ., Morgantown, W.Va. 316 Demain Ave.

Eilenberg, Samuel. Asst. Prof., Univ. of Michigan, Ann Arbor, Mich.

Eisenhart, Churchill. Asst. Prof., Univ. of Wisconsin, Madison, Wis. 1662 Monroe $\mathrm{St}$.

$\dagger$ Eisenhart, L. P. Dean, Princeton Univ., Princeton, N.J.

Elder, Alfonso. Dean, Liberal Arts, North Carolina Coll. for Negroes, Durham, N.C.

Elder, C. C. Hydraulic Engineer, Metropolitan Water District of Southern California, 306 W. 3d St., Los Angeles, Calif.

Ellingson, H. E. Luther Coll., Decorah, Iowa.

Elliott, E. R., Asst. Prof., Hampden-Sydney Coll., Hampden-Sydney, Va. 906 Buford St., Corpus Christi, Tex.

Elliott, W. W. Prof., Duke Univ., Durham, N.C. 4721 Duke Station.

Ellis, Dr. G. A. Lawyer, 120 Broadway, New York, N.Y.

Ellis, J. R. Booker T. Washington High School, Tulsa, Okla. 1110 E. Pine St. §Ellis, Wade. 921 Woodlawn, Ann Arbor, Mich.

Elston, J. S. Asst. Actuary, Travelers Ins. Co., Hartford, Conn.

Elveback, Mary L. Hunter Coll., 695 Park Ave., New York, N.Y.

$\S$ Embree, R. S. Asst., Univ. of Wisconsin, Madison, Wis.

Emch, Arnold. Prof. Emeritus, Univ. of Illinois, Urbana, Ill. 1002 S. Orchard St.

Engstrom, H. T. Asso. Prof., Yale Univ., New Haven, Conn. Lt. Com., U.S.N.R., U.S. Navy Dept., Washington, D.C. 406 N. Thomas St., Arlington, Va.

Epstein, Dr. Benjamin. Physicist, Ballistic Lab., Frankford Arsenal, Philadelphia, Pa. 4721 Leiper St.

§Epstein, Dr. Marion G. (Mrs. Jess). 132 W. Maple Ave., Moorestown, N.J. 
Erdös, Dr. Paul. Univ. of Pennsylvania, Philadelphia, Pa. Bennett Hall.

Erickson, Dr. R. W. Instr., Hibbing Junior Coll., Hibbing, Minn.

Erickson, W. S. Instr., St. Olaf Coll., Northfield, Minn.

Erkiletian, D. H. Instr., School of Mines and Metallurgy, Univ. of Missouri, Rolla, Mo.

$\dagger$ Escott, E. B. Actuary. 1019 S. East Ave., Oak Park, Ill.

$\S$ Escott, F. H. R.F.D. 1, Bridgeport, Conn.

Esty, T. C. Prof. Emeritus, Amherst Coll., Amherst, Mass. First National Bank.

Ettlinger, H. J. Prof., Univ. of Texas, Austin, Tex. 3110 Harris Park Ave.

$\dagger$ Evans, G. C. Prof., Univ. of California, Berkeley, Calif. Wheeler Hall.

Evans, G. W. 16 Beverly Road, Swampscott, Mass.

Evans, H. B. Prof. Emeritus, Univ. of Pennsylvania, Philadelphia, Pa. 88 Merbrook Lane, Merion Station, $\mathrm{Pa}$.

Evans, H. P. Prof., Univ. of W.isconsin, Madison, Wis. North Hall.

Evans, J. O. Consulting Aeronautical Engineer, 558 14th St. S.E., Washington, D.C.

Everett, Dr. C. J. Instr., Univ. of Wisconsin, Madison, Wis. 2554 Kendall Ave.

Everett, H. S. Prof., Univ. of Chicago, Chicago, Ill.

$\S$ Ewing, Frances E. Asst., Univ. of Illinois, Urbana, I11. 710 Nevada St.

Ewing, G. M. Asst. Prof., Univ. of Missouri, Columbia, Mo. 213 Engineering Bldg.

Ewing, (W.) M. Asso. Prof. of Geophysics, Lehigh Univ., Bethlehem, Pa. Asso. in Submarine Geology, Woods Hole Oceanographic Inst., Woods Hole, Mass.

Falconer, Dr. B. L. 315 Ward St., Marlin, Tex.

Falvey, Frances E. Ward-Belmont School, Nashville, Tenn.

Fan, Dr. S. C. Care of L. C. Chia, Lane 483-56 Yu Yuan Road, Shanghai, China.

§Farley, B. G. Radiation Lab., Massachusetts Inst. of Tech., Cambridge, Mass.

Farnau, E. F. Prof., Chemistry Dept., Univ. of Cincinnati, Cincinnati, Ohio.

Farrell, O. J. Asso. Prof., Union Coll., Schenectady, N.Y. Stone Ridge Road, R.F.D. 1.

Faulkner, Donald. Prof., John B. Stetson Univ., De Land, Fla. 335 W. Wisconsin Ave.

Fay, E. A. Central Y.M.C.A., Rochester, N.Y.

Fazel, Dr. C. S. Chief of Research, Nitrogen Div., The Solvay Process Co., Hopewell, Va.

Feather, E. H. Capt., U.S.A., retired. Prof., Valley Forge Military Junior Coll., Wayne, Pa. $526 \mathrm{~W}$. Beechtree Lane.

Fejér, Lipót. Prof., Univ. of Budapest, Budapest, Hungary. Mathematical Seminary VIII, Muzeum-Körut 6-8.

Feld, Dr. J. M. Instr., Queens Coll., Flushing, N.Y. Care of Miss Helen Kogut, 751 St. Marks Ave., Brooklyn, N.Y. U.S.A.

Feller, W. K. Asso. Prof., Brown Univ., Providence, R.I.

$\S$ Fenichel, S. A. 301 S. Illinois St., Belleville, I11.

Fenn, I. H. Prof., Polytech. Inst. of Brooklyn, Brooklyn, N.Y.

$\S$ Ferguson, W. A. Roanoke, Mo. Lt., U.S.A., Engineer Replacement Training Center, Camp Claiborne, La.

Ferguson, W. E. Instr., Univ. of Missouri, Columbia, Mo. 511 S. 6th St.

Fernbach, Sidney. Junior Physicist, Ballistic Lab., Frankford Arsenal, Philadelphia, Pa. 1011 N. 6th St.

$\dagger$ Ferry, F. C. Pres. Emeritus, Hamilton Coll., Clinton, N.Y. 324 Hart St., New Britain, Conn.

§Fialkow, Dr. A. D. Lecturer, School of Pharmacy, Columbia Univ., New York, N.Y. 1755 Weeks Ave., Bronx. Instr., Defense Training Inst., 375 Pearl St., Brooklyn, N.Y.

Ficken, F. A. Prof., Univ. of Tennessee, Knoxville, Tenn.

Field, Peter. Prof., Univ. of Michigan, Ann Arbor, Mich. 904 Olivia Ave.

Finan, E. J. Asso. Prof., Catholic Univ. of America, Washington, D.C. 604 Girard St. N.E.

§Finch, J. V. 1226 Sweet Briar Road, Shorewood Hills, Madison, Wis. 
Findlay, William. Prof., McMaster Univ., Hamilton, Ont., Canada. 131 Chedoke Ave.

§Fine, N. J. Instr., Purdue Univ., Lafayette, Ind.

§Finkel, B. F. Prof. Emeritus, Drury Coll., Springfield, Mo. N. Clay Ave.

Fischer, C. H. Asst. Prof., Univ. of Michigan, Ann Arbor, Mich. 3016 Angell Hall.

Fisher, Dr. F. G. Instr., Univ. of Santa Clara, Santa Clara, Calif.

Fisher, H. A. Prof., North Carolina State Coll., Raleigh, N.C. State College Station.

$\dagger$ Fiske, T. S. Prof. Emeritus, Columbia Univ., New York, N.Y. 2 Oak Crescent, Poughkeepsie, N.Y.

$\dagger$ Fite, Dr. W. B. Retired. Columbia Univ., New York, N.Y.

Fithian, J. H. Prof., Newark Coll. of Engineering, Newark, N.J. 157 Halsted St., East Orange, N.J.

Fitterer, J. C. Prof., Colorado School of Mines, Golden, Colo. 1620 Maple St.

Fitzpatrick, J. D. Instr., Creighton Univ., Omaha, Neb. 2102 W. Wisconsin Ave., Milwaukee, Wis.

Flanders, D. A. Asso. Prof., New York Univ., University Heights, New York, N.Y. Bedford Road, Chappaqua, N.Y.

Fleiger, A. G. Research Engineer, Detroit Edison Co., Detroit, Mich. 13193 Wisconsin St.

Flexner, W. W. Asso. Prof., Cornell Univ., Ithaca, N.Y. 109 Iroquois Road.

Flood, Dr. M. M. Research Asso., Princeton Univ., Princeton, N.J. 20 Nassau St. Official Investigator, National Defense Research Com.

Focke, T. M. Dean, Case School of Applied Science, Cleveland, Ohio. 10900 Euclid Ave.

Folley, K. W. Asso. Prof., Wayne Univ., Detroit, Mich.

$\S$ Folz, C. H. Asst. Actuary, Western and Southern Life Ins. Co., 4th and Broadway, Cincinnati, Ohio.

Foraker, F. A. Prof., Univ. of Pittsburgh, Pittsburgh, Pa. 1313 Macon Ave.

Ford, L. R. Prof., Illinois Inst. of Tech., Chicago, Ill.

$\dagger$ Ford, Dr. W. B. Retired. Hayts Corners, N.Y.

Forder, H. G. Prof., University Coll., Auckland, New Zealand.

Forrest, J. H. Prof., Langston Univ., Langston, Okla. Box 185.

Forsyth, C. H. Prof., Dartmouth Coll., Hanover, N.H.

§Forsythe, Alexandra I. (Mrs. G. E.). Research Dept., Douglas Aircraft Co., E1 Segunda, Calif. 555 Kelton Ave., West Los Angeles, Calif.

Forsythe, Dr. G. E. 1819 Hill St., Ann Arbor, Mich. U.S.A., Air Corps.

Fort, Tomlinson. Dean, Graduate School, Lehigh Univ., Bethlehem, Pa.

Foster, A. L. Asst. Prof., Univ. of California, Berkeley, Calif.

Foster, M. C. Prof., Wesleyan Univ., Middletown, Conn. 30 Gordon P1.

Foster, R. M. Be11 Telephone Labs., 463 West St., New York, N.Y. 122 E. Dudley Ave., Westfield, N.J.

Fowler, F. H. Engineer, Propeller Div., Curtiss-Wright Corp., Caldwell, N.J.

Fowler, Sister M. Charlotte. Nazareth Coll., Louisville, Ky.

Fox, A. H. Asst. Prof., Union Coll., Schenectady, N.Y. 1101 Millington Road.

Fox, Mother Ethelyn. Prof., Barat Coll. of the Sacred Heart, Lake Forest, I11.

Fox, Dr. R. H. Asso., Univ. of Illinois, Urbana, I11. 507 S. McCullough St.

Foyle, Grace A. (Mrs. W. R.). 21-45 77th St., Jackson Heights, N.Y.

Frame, J. S. Asso. Prof., Allegheny Coll., Meadville, Pa. 351 Woodland P1.

Frankel, E. T. Asso. Statistician, New York State Dept. of Social Welfare, Albany, N.Y. 491 State St.

$\S$ Frankel, S. P. Univ. of California, Berkeley, Calif.

Franklin, Constance W. (Mrs. Philip). 312 Pleasant St., Belmont, Mass.

Franklin, Philip. Prof., Massachusetts Inst. of Tech., Cambridge, Mass. 312 Pleasant St., Belmont, Mass.

Fraser, Peter. Prof., Univ. of Bristol, Bristol, England.

$\S$ Fraser, Dr. W. C. G. Asst. Gauge Examiner, Gauge Testing Lab., National Research Council, Ottawa, Ont., Canada.

Fréchet, Maurice. Prof., Inst. Henri Poincaré, Univ. of Paris, Paris, France.

Frecheville, George. Agric. Economics Research Inst., Univ. of Oxford, Oxford, England. 56 Holywell St. 
French, A. T. Instr., State Teachers Coll., Winona, Minn.

Frey, E. U. 2962 Decatur Ave., Bronx, New York, N.Y.

Fried, Dr. Hans. Research Asst., Sproul Observ., Swarthmore Coll., Swarthmore, $\mathrm{Pa} .361$ Lancaster Ave., Haverford, $\mathrm{Pa}$.

Friedman, Dr. Bernard. Instr., Wilson Junior Coll., Chicago, I11. 5454 Cornell Ave.

§Friedman, Morris. Instr., Wayne Univ., Detroit, Mich. 3764 Clements Ave.

Friedrichs, K. O. Asso. Prof., New York Univ., New York, N.Y. 39 Glenorchy Road, New Rochelle, N.Y.

Frink, Dr. Aline H. (Mrs. Orrin). Instr., Pennsylvania State Coll., State College, Pa. 706 Sunset Road.

Frink, Orrin. Prof., Pennsylvania State Coll., State College, Pa. 706 Sunset Road.

Fry, Dr. T. C. Mathematical Research Dir., Bell Telephone Labs., 463 West St., New York, N.Y.

Fry, W. J. Physicist, Sound Div., Research Lab., U. S. Navy Dept., Washington, D.C.

§Fubini, Dr. Guido. Inst. for Advanced Study, Princeton, N.J.; Lecturer, New York Univ., New York, N.Y. 88 Central Park W., New York, N.Y.

Fudge, Dr. Helen G. Holmes Junior High School, Philadelphia, Pa. Rosemont, $\mathrm{Pa}$.

Fuller, Dr. D. L. Physical Chemist, Shell Development Co., Emeryville, Calif.

\$Fullerton, R. E. Asst., Yale Univ., New Haven, Conn. 2727 Yale Station.

Fulton, D. G. Asst. Prof., Ohio Northern Univ., Ada, Ohio. 805 S. Main St.

Gaba, M. G. Prof., Univ. of Nebraska, Lincoln, Neb. 2755 Rathbone Road.

Gage, W. H. Asso. Prof., Univ. of British Columbia, Vancouver, B.C., Canada.

Gaines, R. E. Prof., Univ. of Richmond, University of Richmond, Va.

Galbraith, A. S. Asst. Prof., Colby Coll., Waterville, Me. 47 Redington St.

Gale, A. S. Dean Emeritus and Prof., Univ. of Rochester, Rochester, N.Y. River Campus.

Garabedian, C. A. Prof., Wheaton Coll.. Norton, Mass.

Garabedian, H. A. Asst. Actuary, John Hancock Mutual Life Ins. Co., 197 Clarendon St., Boston, Mass.

Garabedian, H. L. Asso. Prof., Northwestern Univ., Evanston, I11.

García, Godofredo. Dean, Univ. of San Marcos, Lima, Peru. Apartado 1979, Casa San Miguel, Bolivar, 280.

§Garcia, Mariano. Fellow, Univ. of Virginia, University Station, Charlottesville, Va. Box 1411.

Gardner, R. S. 711 W. High St., Bryan, Ohio. U.S.A., Air Forces, Wendover Field, Utah.

§Garfin, Dr. Louis. Instr., U.S.A., Air Corps Tech. School, Scott Field, I11. 525 E. Washington, Belleville, Ill.

Garis, C. F. F. Dean and Prof., Union Coll., Schenectady, N.Y.

Garrahan, Sister M. Immaculata. Instr., Coll. Misericordia, Dallas, Pa.

Garrison, Dr. G. N. Instr., Coll. of the City of New York, 139th St. and Convent Ave., New York, N.Y.

Garvin, Sister M. Cleophas. Prof., Science Dept., Notre Dame Coll., South Euclid, Ohio.

Gaskell, Dr. R. E. Fellow, School of Mech., Brown Univ., Providence, R.I. 61 Barnes St.

Gaspar, F. L. Dir., Inst. of Applied Math., and Prof., Univ. of Rosario, Rosario, Argentina. Calle 25 de Diciembre 1433.

§Gassensmith, Rev. F. M. Asso. Prof., Univ. of Notre Dame, Notre Dame, Ind.

Gatewood, B. E. Asst. Prof., Louisiana Polytech. Inst., Ruston, La. Stress Engineer, McDonnell Aircraft Corp., St. Louis, Mo. 9038 McNulty Dr., Overland, St. Louis, Mo.

Gay, H. J. Prof., Worcester Polytech. Inst., Worcester, Mass. 7 Belvidere Ave.

Gehman, H. M. Prof., Univ. of Buffalo, Buffalo, N.Y. 163 Winspear Ave.

§Geiringer, Dr. Hilda P. Visiting Lecturer, Bryn Mawr Coll., Bryn Mawr, Pa.

Gelbart, Dr. Abe. Instr., North Carolina State Coll., Raleigh, N.C. School of Mech., Brown Univ., Providence, R.I. 
Gentry, F. C. Asso. Prof., Louisiana Polytech. Inst., Ruston, La. 2644 Dwight Way, Berkeley, Calif. Asso. Instr., U.S.N. Pre-Flight School, St. Mary's College, Calif.

George, Dr. T. S. Instr., Univ. of Florida, Gainesville, Fla. Lt. (j.g.), U.S.N.R. U.S.S. Sangamon, Care of Postmaster, New York, N.Y.

Gere, Dr. B. H. Arnold, Md. Lt. (j.g.), U.S.N.R., Postgraduate School, U. S. Naval Acad., Annapolis, Md.

Gergen, J. J. Prof., Duke Univ., Durham, N.C. 4771 Duke Station.

Gerhardt, Henry. Merchant. 8 N. Royal St., Mobile, Ala.

Gerst, Rev. F. J. Prof., Loyola Univ., Chicago, I11.

Getchell, B. C. Asst. Prof., Butler Univ., Indianapolis, Ind.

Ghent, K. S. Asst. Prof., Univ. of Oregon, Eugene, Ore.

Gibbens, Gladys. Asst. Prof., Univ. of Minnesota, Minneapolis, Minn. 121 Folwell Hall.

Gibson, J. L. Prof. Emeritus, Univ. of Utah, Salt Lake City, Utah. 1337 Harrison Ave.

§Gibson, R. W. Asst., Univ. of Illinois, Urbana, Ill.

Giddings, H. A. Asso. Prof., Univ. of New Hampshire, Durham, N.H.

Giese, Dr. J. H. Instr., Purdue Univ., Lafayette, Ind.

Gilbarg, Dr. David. Asst. Physicist, Ordnance Lab., Navy Yard, U. S. Navy Dept., Washington, D.C. 2701 14th St. N.W.

Gilbert, Dr. P. W. Instr., Texas Tech. Coll., Lubbock, Tex.

Gilchrist, Lachlan. Asso. Prof., Physics Dept., Univ. of Toronto, Toronto, Ont., Canada.

Gill, B. P. Prof., Coll. of the City of New York, 139th St. and Convent Ave., New York, N.Y. 493 Warwick Ave., West Englewood, N.J.

Gillam, Dr. B. E. Instr., Univ. of Missouri, Columbia, Mo. 208 Engineering Bldg.

Gillespie, William. Prof. Emeritus, Princeton Univ., Princeton, N.J. Fine Hall.

Gillis, Dr. Joseph. Lecturer, Queen's Univ., Belfast, Northern Ireland.

§Gillman, Leonard. Fellow, Columbia Univ., New York, N.Y. 549 Riverside Dr.

Gilman, R. E. Asso. Prof., Brown Univ., Providence, R.I.

§Gingerich, H. F. Asst., Univ. of Illinois, Urbana, I1l. 160 Math. Bldg.

Gingery, w. G. Prin., George Washington High School, Indianapolis, Ind. 2215 W. Washington St.

§Ginsberg, N. M. Fellow, Univ. of Illinois, Urbana, I1l. 306 E. Healey St., Champaign, Ill.

Ginsburg, Jekuthiel. Prof., Yeshiva Coll., New York, N.Y. 610 W. 139th St.

Girshick, M. A. Senior Agric. Statistician, Div. of Statistical and Historical Research, Bureau of Agric. Economics, U. S. Dept. of Agric., Washington, D.C.

Givens, Dr. J. W. Instr., Northwestern Univ., Evanston, I11.

Glass, T. F. Instr., Marion Inst., Marion, Ala.

Glazier, Harriet E. Asst. Prof. Emeritus, Univ. of California at Los Angeles, Los Angeles, Calif. 1307 Lucile Ave.

§Gleason, A. M. 21 Howe P1., Bronxville, N.Y. Enisign, U.S.N.R., 2017 Eye St., Washington, D.C. Arts Club.

Gleason, R. E. Prof., Temple Univ., Philadelphia, Pa. 2022 N. Park Ave.

Glenn, W. H. Instr., Pasadena Junior Coll., Pasadena, Calif. 1425 Beech St., South Pasadena, Calif.

Gleyzal, André. Prof., St. Michael's Coll., Winooski Park, Vt.

Glover, R. E. Senior Engineer, Bureau of Reclamation, U. S. Dept. of Interior, Denver, Colo.

Godfrey, E. L. Instr., Fenn Coll., Cleveland, Ohio.

Gödel, Dr. Kurt. Inst. for Advanced Study, Princeton, N.J.

Goff, J. A. Dean, Towne Scientific School, Univ. of Pennsylvania, Philadelphia, Pa. 109 Engineering Bldg.

Goheen, Dr. H. E. Ensign, U.S.N.R., Midshipmen's School, Northwestern Univ., Chicago, Ill.

Gokhale, V. D. Prof., Univ. of the Philippines, Manila, Philippine Islands.

Gold, J. S. Asso. Prof., Bucknell Univ., Lewisburg, Pa. 306 S. 3d St.

Goldberg, Michael. Engineer, Bureau of Ordnance, U. S. Navy Dept., Washington, D.C. 5823 Potomac Ave. N.W. 
Goldstine, Dr. H. H. Instr., Univ. of Michigan, Ann Arbor, Mich. 1st Lt., U.S.A., Air Forces.

Goldsworthy, E. C. Asst. Prof., Univ. of California, Berkeley, Calif. 201 Admin. Bldg.

Golomb, Dr. Michael. Instr., Purdue Univ., Lafayette, Ind.

Gonzalez, J. M. Asst. Prof., Spring Hill Coll., Spring Hill, Ala.

González-Domínguez, Dr. Alberto. Univ. of Buenos Aires, Buenos Aires, Argentina. Perú 222.

González-Rodríguez, M. O. Asst. Prof., Univ. of Havana, Havana, Cuba. Faculty of Sciences.

Good, R. A. Asst., Univ. of Wisconsin, Madison, Wis. North Hall.

Goodier, J. N. Prof., Sibley School of Mechanical Engineering, Cornell Univ., Ithaca, N.Y. 109 Cayuga Heights Rd.

Gordon, W. O. Asst. Prof., Pennsylvania State Coll., State College, Pa.

Gore, G. D. Prof., Central Y.M.C.A. Coll., 19 S. LaSalle St., Chicago, I11. 1707 Burlington Ave., Downers Grove, Ill.

§Gorn, Dr. Saul. 2390 Tiebout Ave., Bronx, New York, N.Y. U.S.A., Air Forces, Tampa, Fla.

Gottschalk, W. H. Asst., Univ. of Virginia, University Station, Charlottesville, Va. 1229 Wertland St.

Gough, Sister M. de Lellis. Prof., Incarnate Word Coll., San Antonio, Tex.

†Gould, Alice B. Care of Samuel Vaughan, 35 Congress St., Boston, Mass.

Gould, S. H. Asst. Prof., Victoria Coll., Univ. of Toronto, Toronto, Ont., Canada.

Gourin, Dr. Eli. Instr., Univ. Extension, Columbia Univ., New York, N.Y. 33 E. 208th St., Bronx.

Gouwens, Cornelius. Asso. Prof., Iowa State Coll., Ames, Iowa.

Graber, M. E. Prof., Morningside Coll., Sioux City, Iowa.

Grable, E. S. Instr., Univ. of Richmond, University of Richmond, Va.

Graesser, R. F. Prof., Univ. of Arizona, Tucson, Ariz. 1648 E. 5th St.

Graham, P. H. Asso. Dean and Prof., Washington Square Coll., New York Univ., New York, N.Y.

$\dagger$ Grant, Alice A. Ontario Coll. of Education, Toronto, Ont., Canada. 215 College St.

Grant, Dr. Anna M. C. 185 N. Village Ave., Rockville Centre, N.Y.

Grant, H. S. Asst. Prof., Rutgers Univ., New Brunswick, N.J.

§Grau, A. A. Fellow, Univ. of Michigan, Ann Arbor, Mich. 713 North Ave. N.E., Grand Rapids, Mich.

Graustein, Dr. Mary C. (Mrs. W. C.). Radcliffe Coll., Cambridge, Mass. 32 Shepard St.

Graves, C. H. Asst. Prof., Pennsylvania State Coll., State College, Pa. Asso. Educational Statistician, Federal Security Agency, U.S. Office of Education, Washington, D.C. 6201 Broad St., Brookmont, Md.

Graves, G. H. Asso. Prof., Purdue Univ., Lafayette, Ind. 227 S. Grant, West Lafayette, Ind.

Graves, L. M. Prof., Univ. of Chicago, Chicago, I1l. Eckhart Hall.

Graves, W. L. Asst. Prof., Drury Coll., Springfield, Mo.

Gray, Dr. Marion C. Tech. Research, Bell Telephone Labs., 463 West St., New York, N.Y.

Greeley, J. B. Instr., Polytech. Inst. of Brooklyn, Brooklyn, N.Y.

Green, Sister Agnes A. Instr., Immaculate Heart Coll., Los Angeles, Calif. 5515 Franklin Ave.

Green, Dr. F. W. Vice Pres., St. Louis Southwestern Railway Lines, St. Louis, Mo. 6203 Washington Ave.

Green, J. W. Asst. Prof., Univ. of Rochester, Rochester, N.Y. River Campus.

Green, Dr. L. J. Instr., Case School of Applied Science, Cleveland, Ohio.

§Greenberg, H. J. Fellow, School of Mech., Brown Univ., Providence, R.I.

\$Greenspan, Bernard. Instr., Brooklyn Coll., Brooklyn, N.Y. 2438 E. 26th St.

Greenwald, Lewis. Junior Physicist, U.S.A., Signal Corps, Radar Lab., Rumson, N.J. Box 199 C, R.F.D. 2, Neptune, N.J.

Greenwood, Dr. R. E. Instr., Univ. of Texas, Austin, Tex. U.S. Navy Dept., Washington, D.C. 1374 Harvard St. N.W. 
Greitzer, S. L. 1535 Undercliff Ave., Bronx, New York, N.Y.

Grennan, Dr. Elizabeth B. (Mrs. John). 719 S. 7 th St., Ann Arbor, Mich.

Greville, Dr. T. N. E. Actuarial Mathematician, Bureau of the Census, U. S. Dept. of Commerce, Washington, D.C. 1714 34th St. N.W.

Griffin, F. L. Prof., Reed Coll., Portland, Ore.

Griffin, Harriet. Asst. Prof., Brooklyn Coll., Brooklyn, N.Y. 1575 Brooklyn Ave.

Griffiths, Lois W. Asso. Prof., Northwestern Univ., Evanston, Ill.

† Groat, B. F. Consulting Engineer. 6733 Emlen St., Philadelphia, Pa.

Gross, Dr. G. L. Asst. Prof., Agric. and Mech. Coll. of Texas, College Station, Tex. 13 Faculty Exch.

Grossman, E. A. Actuarial Dept., U.S. Life Ins. Co., 101 5th Ave., New York, N.Y. U.S.A., Aberdeen Proving Ground, $M d$.

§Groth, A. O. Asst. Actuary, Equitable Life Ins. Co. of Iowa, Des Moines, Iowa.

Grove, C. C. Asst. Prof., Coll. of the City of New York, 17 Lexington Ave., New York, N.Y. 143 Milburn Ave., Baldwin, N.Y.

Grove, V. G. Prof., Michigan State Coll., East Lansing, Mich. 438 Rosewood Ave.

§uenther, Dr. P. E. Instr., Case School of Applied Science, Cleveland, Ohio.

Guggenbiihl, Laura. Asst. Prof., Hunter Coll., Bedford Park Blvd. and Navy Ave., New York, N.Y.

Guinand, Dr. A. P. Care of J. F. Turnbull, Bramshott Farm, Orillia, Ont., Canada. Flight Lt., R.C.A.F., Chatham, N.B., Canada.

Gumbel, E. J. Visiting Prof., New School for Social Research, 66 W. 12th St., New York, N.Y. 3820 Waldo Ave.

†Gummere, H. V. Dir., Strawbridge Memorial Observ., Haverford Coll., Haverford, $\mathrm{Pa}$.

Gunder, D. F. Asso. Prof., Colorado State Coll. of Agric. and Mech. Arts, Fort Collins, Colo.

§Gunderson, N. G. Instr., Cornell Univ., Ithaca, N.Y. White Hall.

Gurney, Dr. Margaret. Asso. Economist, Bureau of the Budget, Washington, D.C. 6102 Lombard St., Cheverly, Hyattsville, Md.

Gutzman, Dr. W. W. Instr., Hunter Coll., 695 Park Ave., New York, N.Y.

Hackney, Lilian. Retired. 89 Kingwood St., Morgantown, W.Va.

§Hadamard, Jacques. Prof. Emeritus, Coll. of France and Polytech. School, Paris, France; Visiting Prof., Columbia Univ., New York, N.Y. 523 W. 112th St., New York, N.Y.

Hadley, Laurence. Prof., Purdue Univ., Lafayette, Ind.

Hagen, Beatrice L. Asst. Prof., Pennsylvania State Coll., State College, Pa.

Haight, N. L. No address.

§Hailperin, Theodore. Instr., Cornell Univ., Ithaca, N.Y. White Hall.

Halbert, K. W. Statistician, American Telephone and Telegraph Co., 195 Broadway, New York, N.Y.

§Halfar, Edwin. Asst., State Univ. of Iowa, Iowa City, Iowa. 316A Physics B1dg.

Hall, Dr. D. W. Instr., Brown Univ., Providence, R.I.

Hall, F. C. Instr., Evening Session, Hunter Coll., New York, N.Y. 430 W. 119th St.

Hall, Marshall. Asst. Prof., Yale Univ., New Haven, Conn. 1805 Silliman Coll.

Hall, Dr. N. A. Asst. Admin. Engineer, Vought-Sikorsky Aircraft, Stratford, Conn.

Hall, Philip. Lecturer, King's Coll., Univ. of Cambridge, Cambridge, England.

$\S$ Hallam, Sarah M. Univ. of California, Berkeley, Calif.

Hallett, Dr. W. N. Instr., Maryland State Teachers Coll., Frostburg, Md. 31 Park Ave.

Halmos, Dr. P. R. Asso., Univ. of Illinois, Urbana, I11. 909 W. California St.

Halperin, Israel. Asst. Prof., Queen's Univ., Kingston, Ont., Canada.

Haltiner, G. J. Ensign, U.S.N.R., Inst. of Meteorology, Univ. of Chicago, Chicago, Ill.

Hamilton, H. J. Asst. Prof., Pomona Coll., Claremont, Calif. 451 W. 7th.

Hamilton, J. A. Actuarial Div., Metropolitan Life Ins. Co., 1 Madison Ave., New York, N.Y.

Hamilton, O. H. Asso. Prof., Oklahoma Agric. and Mech. Coll., Stillwater, Okla. 503 Pine St. Lt., U.S.N.R. 
Hammer, Dr. Hans-Karl. Research Lab., Texas Co., Beacon, N.Y. Instr. in German, U.S. Military Acad., West Point, N.Y.

Hammer, Dr. P. C. Instr., Oregon State Coll., Corvallis, Ore.

\$Hamming, Dr. R. W. Instr., Univ. of Illinois, Urbana, I1l. 359 Math. B1dg.

Hammond, E. S. Dir. of Admissions and Prof., Bowdoin Coll., Brunswick, Me. 9 Thompson St.

Hancock, Harris. Prof. Emeritus, Univ. of Cincinnati, Cincinnati, Ohio. University Station, Charlottesville, Va.

Hansman, Dr. Margaret M. Instr., Colorado Coll., Colorado Springs, Colo. 2038 Armstrong Ave.

Hanson, E. H. Prof., North Texas State Teachers Coll., Denton, Tex. 619 W. Oak St.

Hanson, W. T. Asst. Prin., Tech. High School, Atlanta, Ga. R.F.D. 1, Smyrna, Ga.

Hardy, G. H. Prof., Trinity Coll., Univ. of Cambridge, Cambridge, England.

†Hardy, Dr. J. G. Retired. Box 32, Williamstown, Mass.

Harkin, D. C. 3000 Crest Ave., Cheverly, Hyattsville, Md.

Harrington, C. H. Instr., Glendale Junior Coll., Glendale, Calif. 1330 Hillside Dr.

Harrington, Dr. W. J. Instr., Pennsylvania State Coll., State College, Pa.

Harris, V. C. 2517 Greenwood, Wilmette, Ill. Ensign, U.S.N.R., Great Lakes, Ill.

$\S$ Harrison, Gerald. Fellow, California Inst. of Tech., Pasadena, Calif.

Harrison, M. G. Math. Consultant, Aerodynamics Group, Republic Aviation Corp., Farmingdale, N.Y. 94 Bayview, Port Washington, N.Y.

Harrold, O. G. Asst. Prof., Louisiana State Univ., University, La.

Harshbarger, Frances. Asso. Prof., Kent State Univ., Kent, Ỏhio.

Hart, W. L. Prof., Univ. of Minnesota, Minneapolis, Minn. 1955 E. River Ter.

Hart, W. W. Retired. 139 Tudor P1., Kenilworth, Ill.

Harter, G. A. Prof. Emeritus, Univ. of Delaware, Newark, Del. 4577 Boston Post Road, Pelham, Manor, N.Y.

Hartman, Dr. Philip. Instr., Queens Coll., Flushing, N.Y. 1st Lt., A.U.S., Field Artillery School, Fort Sill, Okla.

Hartnell, George. Retired. Wyoming, N.Y.

Hartung, M. L. Asst. Prof., Univ. of Chicago, Chicago, I11. Graduate Education Bldg.

Hartzler, H. H. Asso. Prof., Goshen Coll., Goshen, Ind.

Harvey, G. G. Asst. Prof., Physics Dept., Massachusetts Inst. of Tech., Cambridge, Mass.

Harvey, W. R. Tech. Staff, Bell Telephone Labs., 463 West St., New York, N.Y.

Haskins, C. N. Prof., Dartmouth Coll., Hanover, N.H. Hanover Road, Lebanon, N.H.

Haskins, E. E. Asso. Prof., Northeastern Univ., Boston, Mass. 213 Jackson St., Newton Centre, Mass.

Hassler, J. O. Prof., Univ. of Oklahoma, Norman, Okla. 425 Lahoma Ave.

Hastings, Cecil. Shelton Hotel, Lexington Ave. and 49th St., New York, N.Y.

Hatcher, T. W. Prof., Virginia Polytech. Inst., Blacksburg, Va.

$\S$ Hattan, Corinne. Asst., Univ. of Illinois, Urbana, I1l. 153 Math. Bldg.

Hausman, B. A. Prof., Univ. of Detroit, Detroit, Mich.

Haviland, E. K. Prof., Lincoln Univ., Lincoln University, Pa. Box 382, Oxford, $\mathrm{Pa}$.

Hawkes, H. E. Dean, Columbia Coll., Columbia Univ., New York, N.Y.

Hawkins, Ernest. Asso. Prof., U. S. Naval Acad., Annapolis, Md. 4 Steele Ave. Hay, G. E. Asst. Prof., Univ. of Michigan, Ann Arbor, Mich.

Hayden, Camilla. Prof., St. Mary-of-the-Woods Coll., St. Mary-of-the-Woods, Ind.

Hayes, Dr. C. A. U.S.A. Engineers, 74 New Montgomery St., San Francisco, Calif. 1417 Dolores St.

Hayes, J. J. Instr., Univ. of Utah, Salt Lake City, Utah.

Haynes, Dr. Nola A. (Mrs. E. S.). 1408 Rosemary Lane, Columbia, Mo.

\$Hayward, Nathan. Engineer, 12 S. 12th St., Philadelphia, Pa.

Hazard, C. T. Prof., Purdue Univ., Lafayette, Ind.

Hazard, Dr. Katherine E. Instr., Wellesley Coll., Wellesley, Mass.

Hazeltine, (L.) A. Prof., Physics Dept., Stevens Inst. of Tech., Hoboken, N.J. 
$\dagger$ Hazlett, Olive C. Asso. Prof., Univ. of Illinois, Urbana, Ill. 574 Station A, Champaign, I11.

H'Doubler, Dr. F. T. R.F.D. 7, Springfield, Mo.

Heaslet, M. A. Asso. Prof., San Jose State Coll., San Jose, Calif.

Hebbert, Dr. C. M. Tech. Staff, Bell Telephone Labs., 463 West St., New York, N.Y.

Hedberg, E. A. Asso. Prof., Baylor Univ., Waco, Tex. Asso. Instr., U.S.N. PreFlight School, Athens, Ga. 180 Milledge Circle.

Hedge, L. B. Asst. Prof., The Citadel, Charleston, S.C. Capt., U.S.A., Ordnance Dept., Kansas Ordnance Plant, Parsons, Kan.

Hedlund, G. A. Prof., Univ. of Virginia, University Station, Charlottesville, Va. 1834 Fendall Ave.

Hedrick, Dr. E. R. Retired. Univ. of California at Los Angeles, Los Angeles, Calif.

Hefner, R. A. Prof., Georgia School of Tech., Atlanta, Ga. Faculty Exch.

Heins, A. E. Asst. Prof., Purdue Univ., Lafayette, Ind. Radiation Lab., Massachusetts Inst. of Tech., Cambridge, Mass.

$\S$ Heins, M. H. Asst. Prof., Tilinois Inst. of Tech., Chicago, Ill.

$\S$ Heller, G. S. Radiation Lab., Massachusetts Inst. of Tech., Cambridge, Mass.

Hellinger, Dr. E. D. Lecturer, Northwestern Univ., Evanston, I11. 2215 Maple Ave.

Helly, Dr. Edward. Instr., Monmouth Junior Coll., Long Branch, N.J. 369 Broadway.

Helmer, Dr. Olaf. Instr., Coll. of the City of New York, New York, N.Y. 496 Hudson St.

$\S$ Helton, F. F. Asst., Univ, of Illinois, Urbana, I11. 153 Math. Bldg.

$\S$ Hemmingsen, Erik. Asst. Instr., Univ. of Pennsylvania, Philadelphia, Pa. 125 S. 37th St. Junior Physicist, Navy Yard, U. S. Navy Dept., Philadelphia, Pa.

Hendrickson, Dr. Morris. Instr., Bowling Green State Univ., Bowling Green, Ohio.

Hennel, Cora B. Prof., Indiana Univ., Bloomington, Ind. 410 S. Park Ave.

$\S$ Herman, J. R. Asso. Actuary, Metropolitan Life Ins. Co., 1 Madison Ave., New York, N.Y.

Herpel, Coleman. Asst. Prof., Undergraduate Center, Pennsylvania State Coll., Hazleton, Pa.

Herr, Gertrude A. Asso. Prof., Iowa State Coll., Ames, Iowa. 2404 $1 / 2$ Knapp St.

Herriot, Dr. J. G. Instr., Stanford Univ., Stanford University, Calif.

Herschfeld, Aaron. Junior Actuarial Mathematician, Office of the Actuary, U. S. Social Security Board, Washington, D.C.

Herzberger, Dr. Max. Research Lab., Eastman Kodak Co., Rochester, N.Y.

Herzog, Dr. Fritz. Instr., Cornell Univ., Ithaca, N.Y. White Hall.

Hess, G. W. Asso. Prof., Howard Coll., Birmingham, Ala. 8009 4th Ave. S.

Hestenes, Dr. A. D. Instr., Carnegie Inst. of Tech., Pittsburgh, Pa.

Hestenes, M. R. Asst. Prof., Univ, of Chicago, Chicago, Ill. Eckhart Hall.

Hett, J. H. Asst. Prof., Manhattan Coll., New York, N.Y. 3140 Netherland Ave., Bronx.

$\S$ Hewitt, Dr. Edwin. Instr., Harvard Univ., Cambridge, Mass. Kirkland House.

Heyda, Dr. J. F. Instr., Michigan State Coll., East Lansing, Mich.

Hickman, J. S. Instr., Rochester Junior Coll., Rochester, Minn.

Hicks, H. C. Asst. Prof., Carnegie Inst. of Tech., Pittsburgh, Pa. 844 Country Club Dr. 16. Aeronautical Engineer, Waco Aircraft Co., Troy, Ohio.

Hickson, A. O. Asst. Prof., Duke Univ., Durham, N.C.

Higdon, Archie. Asst. Prof., Theoretical and Applied Mech. Dept., Iowa State Coll., Ames, Iowa. Maj., U.S.A., Air Corps, Jefferson Barracks, Mo.

Highberg, I. E. Asst. Prof., Whitman Coll., Walla Walla, Wash.

Hightower, Ruby U. Prof., Shorter Coll., Rome, Ga.

$\S$ Hildebrand, Dr. F. B. Instr., Massachusetts Inst. of Tech., Cambridge, Mass.

Hildebrandt, E. H. C. Asst. Prof., New Jersey State Teachers Coll., Upper Montclair, N.J.

Hildebrandt, T. H. Prof., Univ. of Michigan, Ann Arbor, Mich. 1930 Cambridge Road.

Hildner, R. C. Asso. Prof., Mt. Union Coll., Alliance, Ohio. 
Hill, C. G. 28 Scott St., Chicago, I11.

Hill, E. L. Asso. Prof., Physics Dept., Univ. of Minnesota, Minneapolis, Minn.

Hill, J. D. Asst. Prof., Michigan State Coll., East Lansing, Mich.

Hill, L. S. Asso. Prof., Hunter Coll., 695 Park Ave., New York, N.Y.

Hill, Sister M. Laetitia. Prof., Our Lady of the Lake Coll., San Antonio, Tex.

Hille, Einar. Prof., Yale Univ., New Haven, Conn. 125 Armory St., Hamden, Conn.

$\S$ Hillman, Abraham. Asst. Mathematician, Mathematical Tables Project, National Bureau of Standards, U. S. Dept. of Commerce, 70 Columbus Ave., New York, N.Y. 3021 Ave. I, Brooklyn, N.Y.

Hinds, Frances C. (Mrs.). Asst. Prof., George Pepperdine Coll., 1121 W. 79th St., Los Angeles, Calif.

Hinrichsen, J. J. L. Asso. Prof., Iowa State Coll., Ames, Iowa.

Hobbs, A. W. Dean, Coll. of Arts and Sciences, Univ. of North Carolina, Chape1 Hill, N.C.

Hochschild, Dr. G. P. 730 Ft. Washington Ave., New York, N.Y. U.S.A., Air Forces, Tampa, Fla.

Hodell, L. R. Research Supervisor, Carter Oil Co., Box 801, Tulsa, Okla.

Hodge, F. H. Asst. Prof. Emeritus, Purdue Univ., Lafayette, Ind. 820 N. Main St., West Lafayette, Ind.

Hoel, P.'G. Asst. Prof., Univ. of California at Los Angeles, Los Angeles, Calif.

Hoersch, Dr. V. A. Asso., Univ. of Illinois, Urbana, I11. 909 S. 1st St., Champaign, I11.

Hoffmann, Banesh. Asst. Prof., Queens Coll., Flushing, N.Y. 149-30 Hollywood Ave.

Hofmann, Dr. Lulu. Box 302, Grass Valley, Calif.

$\S$ Hohaus, R. A. Asst. Actuary, Metropolitan Life Ins. Co., 1 Madison Ave., New York, N.Y.

Hohn, Dr. F. E. Instr., Univ. of Arizona, Tucson, Ariz.

Holgate, T. F. Prof. Emeritus, Northwestern Univ., Evanston, I11. 617 Library P1.

Holl, D. L. Prof., Iowa State Coll., Ames, Iowa. 2323 Donald St.

Holland, Sister M. Charlotte. Prof. and Registrar, St. Francis Xavier Coll. for Women, 4900 Cottage Grove Ave., Chicago, Ill.

Hollcroft, Mary P. (Mrs. T. R.). Aurora, N.Y.

Hollcroft, T. R. Prof., Wells Coll., Aurora, N.Y.

Holley, Dr. J. L. 1318 L St. N.W., Washington, D.C.

Holly, Melita A. Research Mathematician, Ordnance Lab., Navy Yard, U.S. Navy Dept., Washington, D.C. 2139 R St. N.W.

Holmes, C. T. Asso. Prof., Bowdoin Coll., Brunswick, Me.

Hooper, Dorothy S. (Mrs. W. J.). Asst. Prof., Principia Coll., Elsah, I11.

Hopf, Eberhard. Prof., Univ. of Leipzig, Leipzig, Germany. Talstrasse 35.

Hopkins, L. A. Asso. Prof., Univ. of Michigan, Ann Arbor, Mich.

Hopkins, Dr. Margarete W. (Mrs.). 196 Clinton Ave., Brooklyn, N.Y.

Hopper, F. F. Dir., New York Public Lib., 476 5th Ave., New York, N.Y.

Hopper, Grace M. (Mrs. V. F.), Asst. Prof., Vassar Coll., Poughkeepsie, N.Y.

Hopwood, Muriel A. Kauai High School, Lihue, Kauai, Hawaii.

$\S$ Horn, Alfred. Asst., Univ. of California, Berkeley, Calif.

Hornberger, H. J. Actuary, Great Northern Life Ins. Co., 110 S. Dearborn St., Chicago, Ill.

Horsfall, I. O. Dir., Extension Div., and Prof., Univ. of Utah, Salt Lake City, Utah.

Horst, Dr. A. P. Supervisor, Selection Research, Procter and Gamble Co., Cincinnati, Ohio.

Horton-Porter, Goldie. See Porter, Goldie Horton.

Horvay, Dr. Gabriel, Instr., Univ. of Cincinnati, Cincinnati, Ohio. Asst. Physicist, David Tavlor Model Basin, U.S. Navy Dept., Carderock, Md. 13 McKay Circle, Bethesda, Md.

Hosford, H. M. Dean, Coll. of Arts and Sciences, Univ. of Arkansas, Fayetteville, Ark. 722 W. Maple.

Hostetter, I. M. Asst. Prof., Oregon State Coll., Corvallis, Ore.

Hotelling, Harold. Prof., Columbia Univ., New York, N.Y. Mountain Lakes, N.J.

Hotelling, Susanne E. (Mrs. Harold). Mountain Lakes, N. J. 
Householder, Dr. A. S. Research Asso. in Biophysics, Univ. of Chicago, Chicago, I11. 644 Euclid Ave., Glen E1lyn, I11.

Houston, W. V. Prof., Physics Dept., California Inst. of Tech., Pasadena, Calif. Div. of National Defense Research, Columbia Univ., New York, N.Y. 60 Gramercy Park.

Hove, Ethel M. Asso. Prof., Nebraska State Teachers Coll., Wayne, Neb. 416 Main St.

Howard, Awghtum S. (Mrs.). Fellow, Univ. of Kentucky, Lexington, Ky. 121 McVey Hall.

Howland, L. A. Prof., Wesleyan Univ., Middletown, Conn.

Hoy, E. A. Asst. Prof., Univ. of Hawaii, Honolulu, Hawaii. 509 W. 121st St., New York, N.Y.

Hoy, Ruth Y. (Mrs. E. A.). Instr., Univ. of Hawaii, Honolulu, Hawaii. 509 W. 121 st St., New York, N.Y.

Hoyt, R. S. Tech. Staff, Bell Telephone Labs., 463 West St., New York, N.Y.

Hsï, Dr. H. Y. Lecturer, Yenching Univ., Peking, China.

Hubbs, H. N. Prof., Hobart Coll., Geneva, N.Y.

Hubert, W. G. Asso. Prof., Coll. of the City of New York, 139th St. and Convent Ave., New York, N.Y.

Hudson, Dr. C. M. Junior Physicist, Ballistic Lab., Frankford Arsenal, Philadelphia, Pa. 1052 Granite St.

Huff, w. N. Instr., The Hill School, Pottstown, Pa.

Hughes, H. K. Asso. Prof., Purdue Univ., Lafayette, Ind.

Hull, Evelyn M. Office Manager, American Mathematical Society, 531 W. 116th St., New York, N.Y.

Hull, Ralph. Prof., Univ. of British Columbia, Vancouver, B.C., Canada.

Hume, Alfred. Chancellor Emeritus and Prof., Univ. of Mississippi, University, Miss.

Humm, Dr. D. G. Personnel Specialist, 416 W. 8th St., Los Angeles, Calif.

Hummel, P. M. Asst. Prof., Univ. of Alabama, University, Ala. Box 1251 .

Humphreys, Mabel G. Asst. Prof., Newcomb Coll., Tulane Univ., New Orleans, La.

Humphreys, W. J. Prof. Emeritus, George Washington Univ., Washington, D.C. Cosmos Club.

Hunt, G. H. Asst. Prof., Univ. of California at Los Angeles, Los Angeles, Calif.

Hunt, Mildred. Prof., Illinois Wesleyan Univ., Bloomington, Il1.

Hunter, Louise S. (Mrs.). Asst. Prof., Virginia State Coll., Ettrick, Va.

$\S$ Hunter, Dr. R. G. Actuary, Equitable Life Ins. Co. of Iowa, Des Moines, Iowa.

$\nmid$ Huntington, E. V. Prof. Emeritus, Harvard Univ., Cambridge, Mass. 48 Highland St.

Huntley, H. B. Mathematician, Research and Development Dept., Cox and Stevens Aircraft Corp., Box 30, Mineola, N.Y.

Hurd, C. C. Asst. Prof., Michigan State Coll., East Lansing, Mich. Lt. (j.g.), U.S.C.G.R., U.S. Coast Guard Acad., Nere London, Conn.

Hurewicz, Witold. Asst. Prof., Univ. of North Carolina, Chapel Hill, N.C. Box 127.

Hurry, J. A. Prof., Physics Dept., San Antonio Junior Coll., San Antonio, Tex.

Hurst, J. W. Asso. Prof., Montana State Coll., Bozeman, Mont. 522 S. 6th.

Hurst, Mary J. 1105 N. Broad St., Galesburg, I11.

Hurt, J. T. Asst. Prof., Agric. and Mech. Coll. of Texas, College Station, Tex.

$\dagger$ Hurwitz, W. A. Prof., Cornell Univ., Ithaca, N.Y. White Hall.

Huston, R. E. Asst. Prof., Rensselaer Polytech. Inst., Troy, N.Y. 4 Broadview Ter., S. Lake Ave.

Hutcherson, W. R. Prof., Berea Coll., Berea, Ky.

Hutchings, W. L. Asso. Prof., Rollins Coll., Winter Park, Fla. 357 Knowles Ave.

Hutchinson, C. A. Prof., Univ. of Colorado, Boulder, Colo.

Hutchinson, J. D. Asso. Prof., Univ. of Houston, Houston, Tex. 2904 Milam St.

Hutchinson, Dr. L. C. Fellow, School of Mech., Brown Univ., Providence, R.I.

Hyers, Dr. D. H. Fellow, Mech. Engineering Dept., California Inst. of Tech., Pasadena, Calif. 101 N. Hill Ave. 
Ingersoll, B. M. 1st Lt., A.U.S., Instr., U.S. Military Acad., West Point, N.Y.

Ingham, A. E. Lecturer, King's Coll., Univ. of Cambridge, Cambridge, England. Ingraham, M. H. Dean, Coll. of Letters and Science, and Prof., Univ. of Wisconsin, Madison, Wis. South Hall.

Ingram, W. H. 150 Claremont Ave., New York, N.Y.

Irwin, Frank. Asso. Prof. Emeritus, Univ. of California, Berkeley, Calif. 2921 Regent St.

§Irwin, P. C. Asso. Actuary, Equitable Life Ins. Co. of Iowa, Des Moines, Iowa.

Isaacs, Dr. R. P. Analytic Engineer, Hamilton Standard Propellers, United Aircraft Corp., East Hartford, Conn.

Iyengar, K. S. K. Prof., Central Coll., Bangalore, India.

Jablon, Seymour. Asst., Columbia Univ., New York, N.Y. 200 W. 108th St.

†Jackson, Dunham. Prof., Univ. of Minnesota, Minneapolis, Minn. 119 Folwell Hall.

Jackson, J. B. Prof., Univ. of South Carolina, Columbia, S.C. 227 S. Waccamaw Ave.

Jackson, Dr. L. L. Retired. 4335 University Station, Tucson, Ariz.

Jackson, O. B. Seismologist, Shell Oil Co., Tulsa, Okla. Box 638, Graham, Tex.

Jackson, Rosa L. Prof., Alabama Coll., Montevallo, Ala.

Jackson, S. B. Asst. Prof., Univ. of Maryland, College Park, Md.

Jacobsen, R. S. Instr., Luther Coll., Decorah, Iowa. 602 North St.

Jacobson, Nathan. Asso. Prof., Univ. of North Carolina, Chapel Hill, N.C. Box 264. Instr., U.S.N., Pre-Flight School, Chapel Hill, N.C.

Jacobus, Dr. D. S. Stevens Inst. of Tech., Hoboken, N.J. 93 Harrison Ave., Montclair, N.J.

Jaeger, C. G. Prof., Pomona Coll., Claremont, Calif.

Jaeger, Dr. J. C. Lecturer, Univ. of Tasmania, Hobart, Australia.

Jaffe, Dr. Haym. Penn Treaty Junior High School, Philadelphia, Pa. 3800 Chestnut St.

James, Glenn. Asso. Prof., Univ. of California at Los Angeles, Los Angeles, Calif.

§James, R. C. Fellow, California Inst. of Tech., Pasadena, Calif. Box 216, Glendora, Calif.

James, R. D. Prof., Univ. of Saskatchewan, Saskatoon, Sask., Canada.

Janes, W. C. Asst. Prof., Kansas State Coll. of Agric., and Applied Science, Manhattan, Kan.

Jaramillo, Dr. T. J. Actuary, Bureau of Treasury, Manila, Philippine Islands. Box 1045.

Jarnagin, M. P. Asst., Duke Univ., Durham, N.C. 630 Milledge Circle, Athens, Ga.

Jeffery, R. L. Prof., Acadia Univ., Wolfville, N.S., Canada.

Jenkins, E. D. Asso. Prof., Eastern Kentucky State Teachers Coll., Richmond, Ky. 1405 Howard Ave., Utica, N.Y.

Jennings, Dr. S. A. Lecturer, Univ. of British Columbia, Vancouver, B.C., Canada.

Jennings, Walter. Instr., Agric. and Mech. Coll. of Texas, College Station, Tex. Lt. (j.g.), U.S.N.R., U.S. Naval Proving Ground, Dahlgren, Va.

Jensen, C. M. Prof., Kansas Wesleyan Univ., Salina, Kan.

§Jerbert, A. H. Fellow, Univ. of Washington, Seattle, Wash. Philosophy Hall.

Jerbert, A. R. Asso. Prof., Univ. of Washington, Seattle, Wash. 5033 Pullman Ave.

Jessen, Børge. Prof., Royal Tech. Coll., Copenhagen, Denmark. Maltegaardsvej 11, Gentofte, Denmark.

$\dagger$ †offe, S. A. Retired. 515 W. 110th St., New York, N.Y.

Johanson, R. N. Asst. Prof., Bradley Polytech. Inst., Peoria, I11.

John, Fritz. Asso. Prof., Univ. of Kentucky, Lexington, Ky. 569 Webster Ave., New Rochelle, N.Y.

Johns, A. E. Asso. Prof., McMaster Univ., Hamilton, Ont., Canada.

Johnson, Evan. Asst. Prof., Pennsylvania State Coll., State College, Pa. Box 321.

Johnson, Marie M. Asst. Prof., Oberlin Coll., Oberlin, Ohio. 257 W. College St.

§Johnson, P. B. Asst., Univ. of Illinois, Urbana, I11. Civilian Public Service Camp, Glendora, Calif. Box 65. 
Johnson, R. A. Prof., Brooklyn Coll., Brooklyn, N.Y. 3421 80th St., Jackson Heights, N.Y.

Johnson, Dr. R. E. Instr., Univ. of Wisconsin, Madison, Wis.

Johnson, Roberta F. Asst. Prof., Wilson Coll., Chambersburg, Pa.

Johnston, F. E. Prof., George Washington Univ., Washington, D.C.

Jones, A. W. Instr., Yale Univ., New Haven, Conn. Johnson Rd., Woodbridge, Conn.

Jones, B. W. Asso. Prof., Cornell Univ., Ithaca, N.Y. White Hall.

Jones, C. D. 13445 Rosemary, Detroit, Mich.

Jones, F. B. Asst. Prof., Univ. of Texas, Austin, Tex. Research Asso., Underwater Sound Lab., Harvard Univ., Cambridge, Mass. 77 Martin St.

Jones, Dr. G. M. Architect and Civil Engineer. 437 E. Oakwood Blvd., Chicago, I11.

Jones, Harris. Prof., U.S. Military Acad., West Point, N.Y. Col., U.S.A.

Jones, P. C. Bureau of Publication, Bell Telephone Labs., 463 West St., New York, N.Y.

Jones, R. W. Asst. Prof., Univ. of Delaware, Newark, Del. 121 Townsend Road.

Jordan, Dr. H. A. Catholic Univ, of America, Washington, D.C. 2512 Que St. N.W.

Jordan, H. E. Asso. Prof., Univ. of Kansas, Lawrence, Kan. 1600 Kentucky St.

Joseph, J. A. Instr., Civil Engineering Dept., New Mexico Coll. of Agric. and Mech. Arts, State College, N.Mex.

Judd, T. E. R.S.C., Auditing Div., Lafayette Bldg., Washington, D.C.

Justice, H. K. Prof., Coll. of Engineering and Commerce, Univ. of Cincinnati, Cincinnati, Ohio. 927 Kreis Lane.

Kac, Dr. Mark. Instr., Cornell Univ., Ithaca, N.Y.

Kagno, Dr. I. N. 3326 Bainbridge Ave., Bronx, New York, N.Y.

§Kakutani, Shizuo. Asst. Prof., Osaka Imperial Univ., Osaka, Japan.

Kales, Dr. M. L. Univ. of Maine, Orono, Me.

Kalinowski, W. C. St. John's Univ., Collegeville, Minn.

§Kalisch, Dr. G. K. Instr., Univ. of Kansas, Lawrence, Kan.

§Kaplan, Samuel. Fellow, Univ. of Michigan, Ann Arbor, Mich. 1104 Catherine.

Kaplan, Dr. Wilfred. Instr., Univ. of Michigan, Ann Arbor, Mich. 1135 Lincoln Ave.

Kaplansky, Dr. Irving. Instr., Harvard Univ., Cambridge, Mass. 17 Sumner Road.

$\uparrow$ Karapetoff, Vladimir. Prof. Emeritus, Cornell Univ., Ithaca, N.Y. 107 Romaine P1., Leonia, N.J.

§Karlin, Rabbi Meyer. 1890 E. 5th St., Brooklyn, N.Y.

Karnes, H. T. Asst. Prof., Louisiana State Univ., University, La.

Karpinski, L. C. Prof., Univ. of Michigan, Ann Arbor, Mich. 1315 Cambridge Road.

§Karush, Dr. William. Mathematician Geophysical Lab., Carnegie Inst., Washington, D.C. 4844 Western Ave. N.W.

†Kasner, Edward. Prof., Columbia Univ., New York, N.Y. 430 W. 116th St.

Kato, Chosaburo. Asso. Prof., Denison Univ., Granville, Ohio.

Katz, William. Attorney, 70 Pine St., New York, N.Y. 1574 Crotona Park E., Bronx.

Kazarinoff, D. K. Univ. of Michigan, Ann Arbor, Mich. 1515 Cambridge Road.

Kearney, Dora E. Asst. Prof., Iowa State Teachers Coll., Cedar Falls, Iowa.

Keeler, I. F. Prof., North Central Coll., Naperville, I11.

Keffer, Ralph. Actuary, Aetna Life Ins. Co., Hartford, Conn.

Kelley, J. L. Asst. Prof., Univ. of Notre Dame, Notre Dame, Ind. Asso. Mathematician, Ballistic Research Lab., Aberdeen Proving Ground, Md.

Kelley, T. L. Prof., Education Dept., Harvard Univ., Cambridge, Mass. 90 Follen Road, Lexington, Mass.

Kells, L. M. Asso. Prof., U. S. Naval Acad., Annapolis, Md. 23 Thompson St.

§Kemble, E. C. Prof., Physics Dept., Harvard Univ., Cambridge, Mass.

Kemmer, P. H. Col., U.S.A., Air Corps, Wright Field, Dayton, Ohio.

Kempner, A. J. Prof., Univ. of Colorado, Boulder, Colo.

Kendall, Claribel. Asso. Prof., Univ. of Colorado, Boulder, Colo. 1305 Euclid Ave. 
Kenna, Rev. J. H. Asst. Prof., Univ. of Notre Dame, Notre Dame, Ind. Moreau Seminary.

Kennedy, E. C. Asso. Prof., Texas Coll. of Arts and Industries, Kingsville, Tex.

Kennedy, Dr. E. S. Instr., Univ. of Alabama, University, Ala. 1st Lt., U.S.A., Military Intelligence Div., General Staff, U. S. War Dept., Washington, D.C.

Kenney, J. F. Asst. Prof., Univ. of Wisconsin at Milwaukee, Milwaukee, Wis. North Hall, Univ. of Wisconsin, Madison, Wis.

Kennison, L. S. Asst. Prof., Brooklyn Coll., Brooklyn, N.Y. Lt. U.S.N.R., 9 Twin Oak Dr., Hyattsville, $M d$.

Kent, J. R. F. Instr., Univ. of Arkansas, Fayetteville, Ark. 90 Bagot St., Kingston, Ont., Canada. Sub.-Lt., R.C.N.V.R.

Kernaghan, Mother Marie. Prof., Maryville Coll., St. Louis, Mo.

Kershner, Dr. R. B. Asso., Johns Hopkins Univ., Baltimore, Md. 3321 Winterbourne Road.

Kersten, H. J. Asst. Prof., Univ. of Cincinnati, Cincinnati, Ohio.

Ketchum, Dr. Gertrude S. (Mrs. P. W.). 803 W. Illinois St., Urbana, I11.

Ketchum, P. W. Asst. Prof., Univ. of Illinois, Urbana, Ill

†Keyser, C. J. Prof. Emeritus, Columbia Univ., New York, N.Y.

Keyser, Sarah Y. (Mrs. C. J.). Asst. Headmistress, Nightingale-Bamford School, New York, N.Y. 50 Morningside Dr.

Kibbey, Dr. D. E. Instr., Michigan State Coll., East Lansing, Mich.

Kiefer, Dr. Lois. Instr., Univ. of Illinois, Urbana, I11. 153 Math. Bldg.

§Kieval, H. S. Instr., North Carolina State Coll., Raleigh, N.C.

Kimball, Dr. B. F. Senior Statistician, New York State Public Service Com., New York, N.Y. 33 Bogart Ave., Port Washington, N.Y.

Kimball, S. H. Asso. Prof., Univ. of Maine, Orono, Me. 25 Forest Ave.

Kimball, W. S. Prof., Robert Coll., Istanbul, Turkey.

King, F. G. No address.

Kingston, H. R. Prof., Univ. of Western Ontario, London, Ont., Canada.

Kingston, Dr. J. M. Asso., Univ. of Washington, Seattle, Wash.

Kinney, J. M. Prof., Wilson Junior Coll., Chicago, I1l. 8058 Bennett Ave.

Kinsman, A. D. 80 County Road, Ipswich, Mass.

Kiokemeister, Dr. Fred. Instr., Cornell Univ., Ithaca, N.Y.

Kirby, A. R. Lecturer, Fordham Univ., New York, N.Y. 2921 Briggs Ave., Bronx.

Kirby, Mary D. (Mrs. A. R.). Instr., Manhattanville Coll. of the Sacred Heart, New York, N.Y. 2921 Briggs Ave., Bronx.

Kirchen, C. J. U.S. Inspector, Zenith Machine Co., Duluth, Minn. 1101 E. 3d St.

Kiss, S. A. Chemical Engineer. 12 E. 88th St., New York, N.Y.

Kitchens, J. W. Naval Observ., U. S. Navy Dept., Washington, D.C.

Klauber, Dr. L. M. Vice Pres. and General Manager, San Diego Gas and Electric Co., San Diego, Calif. 233 W. Juniper St.

Kleene, S. C. Asso. Prof., Amherst Coll., Amherst, Mass. Lt. (j.g.), U.S.N.R.

Kleinschmidt, R. B. Instr., Coll. of Engineering, Rutgers Univ., New Brunswick, N.J.

Kline, J. R. Prof., Univ. of Pennsylvania, Philadelphia, Pa. 529 Riverview Road, Swarthmore, $\mathrm{Pa}$.

Kline, Dr. Morris. Instr., New York Univ., New York, N.Y. Physicist, U.S.A., Signal Corps, Fort Monmouth, N.J. Care of Wainwright, Little Silver, N.J.

Klipple, E. C. Asso. Prof., Agric. and Mech. Coll. of Texas, College Station, Tex. 107 Faculty Exch.

Knebelman, M. S. Prof., State Coll. of Washington, Pullman, Wash. 2008 Indiana St.

Knedler, P. A. Prof., State Teachers Coll., Kutztown, Pa. East Texas, Pa.

Knipp, J. C. Asst. Prof., Univ. of Pittsburgh, Pittsburgh, Pa. 450 Cathedral of Learning.

§Knobelauch, E. A. Instr., Drew Univ., Madison, N.J.

Knowler, L. A. Asst. Prof., State Univ. of Iowa, Iowa City, Iowa. 212 Physics Bldg.

Knox, R. H. Asst. Prof., Virginia Military Inst., Lexington, Va. 313 Letcher Ave.

Koch, R. F. $2 d$ Lt., U.S.A., Signal Corps, Radar School, Camp Murphy, Fla. $43643 d$ St., West Palm Beach, Fla. 
Koehler, Dr. Fulton. Actuarial Dept., Northwestern National Life Ins. Co., Minneapolis, Minn. R.F.D. 2, Hopkins, Minn.

Koehler, T. L. Asst. Prof., Muhlenberg Coll., Allentown, Pa. 625 N. 24th St.

$\S$ Kolchin, Dr. E. R. U.S.A., Air Forces, Office of Ordnance Officer, Tampa, Fla. 218 Hyde Park Ave.

Kolsrud, E. R. Univ. of Illinois, Urbana. I11. 201 S. Gregory St.

§omm, Horace. Engineering Dept., Curtiss-Wright Corp., Buffalo, N.Y. 399 Jefferson Ave.

Koopman, B. O. Asso. Prof., Columbia Univ., New York, N.Y.

Korgen, R. L. Instr., Bowdoin Coll., Brunswick, Me. 38 College St.

Kormes, Joan P. (Mrs. Mark). Instr., Brooklyn Coll., Brooklyn, N.Y. 119 E. 84th St., New York, N.Y.

Kormes, Dr. Mark. Asst. Dir., New York State Ins. Fund, 625 Madison Ave., New York, N.Y. 119 E. 84th St.

Korn, Arthur. Prof. of Electrical Engineering, Stevens Inst. of Tech., Hoboken, N.J. 523 River St.

Korzybski, Alfred. Dir., Inst. of General Semantics, 1234 E. 56th St., Chicago, I11.

Kossack, C. F. Asst. Prof., Univ. of Oregon, Eugene, Ore.

Kovarik, A. F. Prof., Physics Dept., Yale Univ., New Haven, Conn.

Krabill, Dr. D. M. Instr., Potomac State School, West Virginia Univ., Keyser, W.Va. Ensign, U.S.N.R.

Kraft, Ona. Collinwood High School, Cleveland, Ohio. 11328 Euclid Ave.

Krall, H. L. Asst. Prof., Pennsylvania State Coll., State College, Pa. 1420 N. Nichols St., Arlington, Va.

Kramer-Lassar, Dr. Edna (Mrs. I. B. Lassar). Thomas Jefferson High School, Brooklyn, N.Y. 500 St. John's P1.

Krathwohl, W. C. Dir., Dept. of Educational Tests and Measurements, and Prof., Illinois Inst. of Tech., Chicago, I11.

Kratz, P. H. 1623 W. Westmoreland, Philadelphia, Pa.

Krieger, Cecilia. Asst. Prof., Univ. of Toronto, Toronto, Ont., Canada. 382 Roxton Road.

Kryloff, Dr. Nicolas. St. Novo-Mosto-Vaya N. 17/A, Ufa, U.S.S.R.

Kubis, J. F. Asst. Prof., Fordham Univ., New York, N.Y.

Kubota, Tadahiko. Prof., Tôhoku Imperial Univ., Sendai, Japan. Coll. of Science.

Kuhn, .H. W. Prof., Ohio State Univ., Columbus, Ohio. 1179 Fairview Ave.

Kuniyeda, Motoji. Prof., Tokyo Univ. of Literature and Science, Tokyo, Japan.

$\dagger$ Kuschke, C. G. P. Prof., Univ. of Puerto Rico, Rio Piedras, Puerto Rico. 723 W. 3d St., Los Angeles, Calif.

Kwerel, S. M. Statistician and Economist, National Resources Planning Board, Washington, D.C.

Lackay, Sister M. Pachomia. St. Teresa's Coll., Kansas City, Mo.

Laden, Dr. H. N. Instr., Univ. of Wisconsin, Madison, Wis. Ensign, U.S.N.R.

Laderman, Jack. Asso. Statistician, Chemical Warfare Service, U.S. War Dept., 292 Madison Ave., New York, N.Y. 3224 Bronx Blvd., Bronx.

Ladner, A. C. Asst. Prof., Denison Univ., Granville, Ohio. Box 253.

Ladue, Dr. Mary E. Instr., Barnard Coll., Columbia Univ., New York, N.Y.

LaFon, Gladys Q. (Mrs. J. E.). 609 S. Flood, Norman, Okla.

Lambert, W. D. Chief, Section of Gravity and Astronomy, and Prin. Mathematician, Coast and Geodetic Survey, U. S. Dept. of Commerce, Washington, D.C.

Lampland, Dr. C. O. Astronomer, Lowell Observ., Flagstaff, Ariz. Box 1640.

Lamson, K. W. Asso. Prof., Lehigh Univ., Bethlehem, Pa.

Lancaster, O. E. Asst. Prof., Univ. of Maryland, College Park, Md.

Lanckton, A. L. Box 163, Athens, Greece.

Lanczos, Cornelius. Prof., Purdue Univ., Lafayette, Ind.

Landau, H. G. Coal Research Lab., Carnegie Inst. of Tech., Pittsburgh, Pa. Asso. Statistician, Office of Quartermaster General, U.S. War Dept., Washington, D.C. 2408 20th St. N.E.

Landers, Dr. A. W. Instr., Brooklyn Coll., Brooklyn, N.Y. 108-48 67th Dr., Forest Hills, N.Y. 
Landers, Dr. Mary K. (Mrs. A. W.). Instr., Hunter Coll., Bedford Park Blvd. and Navy Ave., New York, N.Y. 108-48 67th Dr., Forest Hills, N.Y.

Landin, Joseph. Asst., Univ. of Notre Dame, Notre Dame, Ind.

Landry, A. E. Prof., Catholic Univ. of America, Washington, D.C. 3624 13th St. N.E., Brookland, D.C.

$\S L$ andshoff, Rolf. Prof., Coll. of St. Thomas, St. Paul, Minn.

Lane, E. P. Prof., Univ. of Chicago, Chicago, I11.

Lane, R. E. Statistician, Flitcraft, Inc., 613-615 S. Maple Ave., Oak Park, I11.

Lang, Dr. G. B. Instr., Univ. of Florida, Gainesville, Fla.

Langer, R. E. Prof., Univ. of Wisconsin, Madison, Wis. 1802 Jefferson St.

Langhaar, Dr. H. L. Structures Engineer, Consolidated Aircraft Corp., Chula Vista, Calif., R.F.D. 1, Box 31-B-1.

LaPaz, Lincoln. Prof. Ohio State Univ., Columbus, Ohio.

§Lapsley, Janie C. Asst., Univ. of Illinois, Urbana, I1l. 153 Math. B1dg.

Larew, Gillie A. Prof., Randolph-Macon Woman's Coll., Lynchburg, Va.

Larguier, E. H. Instr., Loyola Univ., New Orleans, La. 4425 S. Johnson St.

Larkin, Sister Francis X. Prof., St. Joseph's Coll. for Women, 245 Clinton Ave., Brooklyn, N.Y.

Larrivee, Dr. J. A. Asso. Astronomer, Nautical Almanac Office, U.S. Naval Observ., Washington, D.C.

Larsen, Thorstein. Asso., School of Engineering, Johns Hopkins Univ., Baltimore, $\mathrm{Md}$.

Larus, J. R. Vice Pres. and Actuary, Phoenix Mutual Life Ins. Co., Hartford, Conn.

LaSalle, Dr. J. P. Instr., Massachusetts Inst. of Tech., Cambridge, Mass. 383 Harvard St.

Lasley, J. W. Prof., Univ. of North Carolina, Chapel Hill, N.C. 523 E. Rosemary Lane.

Lass, Harry. Asst., Univ. of California at Los Angeles, Los Angeles, Calif. $2805 \mathrm{r} / 2$ Fairmount St.

Latimer, C. G. Prof., Univ. of Kentucky, Lexington, Ky.

Latshaw, V. V. Asst. Prof., Lehigh Univ., Bethlehem, Pa. 1228 W. North St.

Lawrence, v. S. Asso. Prof., Cornell Univ., Ithaca, N.Y. Maj., U.S.A., U. S. Military Acad., West Point, N.Y.

Lawton, w. S. Asst. Prof., Temple Univ., Philadelphia, Pa. 7500 New 2d St., Melrose Park, Pa.

Leach, C. E. R.F.D. 2, Marion, Ohio.

$\S$ Learson, R. J. Actuary, Western and Southern Life Ins. Co., 4th and Broadway, Cincinnati, Ohio.

Leavens, D. H. Research Asso., Cowles Com., Univ. of Chicago, Chicago, I11.

Leavitt, W. G. Ground School Instr., U. S. Naval Air Station, Corpus Christi, Tex. $11172 d$ St.

LeCaine, Dr. Jeanne. Instr., Smith Coll., Northampton, Mass. Junior Professional, National Research Council, Ottarva, Ont., Canada. 107 Dalhousie St.

Lee, (T.) H. Asst., Univ. of Wisconsin, Madison, Wis. North Hall.

Leech, J. S. Yale Univ., New Haven, Conn. 2737 Yale Station.

Lefschetz, Solomon. Prof., Princeton Univ., Princeton, N.J.

Lehmann, C. H. Instr., School of Engineering, Cooper Union, New York, N.Y. 144-17 29th Ave., Flushing, N.Y. Defense Training Inst., 375 Pearl St., Brooklyn, N.Y.

Lehmer, D. H. Asso. Prof., Univ. of California, Berkeley, Calif. 942 Hilldale Ave.

Lehner, Dr. Joseph. Instr., Cornell Univ., Ithaca, N.Y. White Hall.

Lehr, (A.) Marguerite (M.). Asso. Prof., Bryn Mawr Coll., Bryn Mawr, Pa. Cartref.

Leibler, Dr. R. A. Instr., Purdue Univ., Lafayette, Ind. 560 Aldine, Chicago, I11. Ensign, U.S.N.R.

Leifer, H. R. Admin. Asst., U. S. Dept. of Labor, Pittsburgh, Pa. 5500 Hays St. U.S.A., Ordnance Replacement Training Center, Aberdeen Proving Ground, $M d$.

Leighton, Dr. Walter, Lecturer, Rice Inst., Houston, Tex.

Leimanis, Eugene. Asso. Prof., Univ. of Latvia, Riga, Latvia. Jumaras ielá 11, dz. 10 . 
LeLeiko, Max. Statistician, 547 W. 142d St., New York, N.Y.

$\S$ Lemmer, J. G. Asst. Prof, of Philosophy, West Baden Coll., Loyola Univ., West Baden Springs, Ind.

Lengyel, Dr. B. A. Physics Dept., Coll. of the City of New York, 139th St. and Convent Ave., New York, N.Y. 69 W. 90th St.

†Lennes, N. J. Prof., Montana State Univ., Missoula, Mont.

Lenzen, V. F. Prof., Physics Dept., Univ. of California, Berkeley, Calif.

Leonard, H. B. Prof., Univ. of Arizona, Tucson, Ariz. 4024 University Station.

Lester, Caroline A. Asst. Prof., New York State Coll. for Teachers, Albany, N.Y.

LeStourgeon, Flora E. Asso. Prof., Univ. of Kentucky, Lexington, Ky. 630 Maxwelton $\mathrm{Ct}$.

Leuschner, A. O. Dir. Emeritus, Student's Observ., and Prof. of Astronomy, Univ. of California, Berkeley, Calif. 1816 Scenic Ave.

Levi, Dr. Beppo. Dir., Mathematical Inst., Univ. of Rosario, Rosario, Argentina. San Lorenzo 2133.

§Levi, Dr. Howard. Instr., U.S.N. Pre-Flight School, Iowa City, Iowa.

§Levin, Dr. J. H. 12515 Broadstreet Blvd., Detroit, Mich. $2 d$ Lt., U.S.A., Aberdeen Proving Ground, $M d$.

Levin, Dr. Madeline. Instr., Hunter Coll., 695 Park Ave., New York, N.Y.

Levine, Jack. Asso. Prof., North Carolina State Coll., Raleigh, N.C. 5548 State College Station.

Levinson, Dr. H. C. Treas., L. Bamberger and Co., Newark, N.J.

Levinson, Norman. Asst. Prof., Massachusetts Inst. of Tech., Cambridge, Mass.

Levit, Dr. R. J. Ensign, U.S.N.R., Cruft Lab., Harvard Univ., Cambridge, Mass.

Levitski, Dr. Jakob. Asst., Hebrew Univ., Jerusalem, Palestine. Math. Inst.

Levy, Harry. Asst. Prof., Univ. of Illinois, Urbana, Ill.

Levy, Sophia H. Asso. Prof., Univ. of California, Berkeley, Calif. 453 Wheeler Hall.

§Lewis, Anne L. Fellow, Univ. of Chicago, Chicago, I11.

Lewis, D. C. Asso. Prof,, Univ. of New Hampshire, Durham, N.H. Box 53.

Lewis, F. A. Prof., Univ. of Alabama, University, Ala. Box 1444.

Lewis, Florence P. Prof., Goucher Coll., Baltimore, Md.

$\S$ Lewis, J. V. Asst., Univ. of California, Berkeley, Calif. 24 Wheeler Hall.

Lewis, Dr. P. E. Instr., Oklahoma Agric. and Mech. Coll., Stillwater, Okla.

Lewy, Hans. Asso. Prof., Univ. of California, Berkeley, Calif. 417 Wheeler Hall.

Lieber, H. G. Asst. Prof., Long Island Univ., Brooklyn, N.Y. 258 Clinton Ave.

Lieber, Dr. Lillian R. (Mrs. H. G.). Dir., Galois Inst. of Math., Long Island Univ, Brooklyn, N.Y. 258 Clinton Ave.

§Light, Dr. F. W. Johns Hopkins Univ., Baltimore, Md. 2410 St. Paul St.

$\S$ Lin, C. C. Asst., California Inst. of Tech., Pasadena, Calif. Guggenheim Lab.

Lindquist, Dr. C. B. Instr., U. S. Naval Acad., Annapolis, Md. Lt. (j.g.), U.S.N.

Lindsay, R. B. Prof., Physics Dept., Brown Univ., Providence, R.I.

Lindstrum, Dr. A. O. Instr., Univ. of Wisconsin, Madison, Wis. U.S.A., Air Forces, Tech. Training Detachment, Univ. of Chicago, Chicago, Ill.

Linehan, P. H. Prof., Coll. of the City of New York, New York, N.Y. 346 Convent Ave.

Linfield, B. Z. Asso. Prof., Univ. of Virginia, University Station, Charlottesville, Va. Hilltop Road.

Ling, D. P. 377 Hudson St., New York, N.Y.

Ling, G. H. Dean Emeritus and Prof. Emeritus, Univ. of Saskatchewan, Saskatoon, Sask., Canada. 10 Wanless Crescent, Toronto, Ont., Canada.

Linscheid, H. W. Asst. Prof., Bluffton Coll., Bluffton, Ohio.

§Lintvedt, D. R. 806 N. Spring Ave., Sioux Falls, S.Dak.

Litterick, Dr. W. S. Dir. of Studies, The Peddie School, Hightstown, N.J.

Litzinger, Marie. Prof., Mt. Holyoke Coll., South Hadley, Mass. Mosier St.

Livens, G. H. Prof., University Coll., Cardiff, Wales.

§Livesay, Naomi M. Asst., Univ. of I1linois, Urbana, I11. 153 Math. B1dg.

Livingood, J. N. B. Instr., Gettysburg Coll., Gettysburg, Pa. 152 Spruce St., Birdsboro, $\mathrm{Pa}$.

Locke, L. L. 950 St. John's P1., Brooklyn, N.Y.

§Lockhart, B. J. Asst., Univ. of Illinois, Urbana, I11. 153 Math. Bldg.

Loewner, Charles. Asst. Prof., Univ. of Louisville, Louisville, Ky. 125 W. Lee St. 
Logsdon, Mayme I. (Mrs.). Asso. Prof., Univ. of Chicago, Chicago, I11.

Long, T. R. Asst. Prof., Univ. of Rochester, Rochester, N.Y. 75 Cypress St.

Longenecker, J. V. S. Farmers and Bankers Life Ins. Co., Wichita, Kan.

Longley, W. R. Prof., Yale Univ., New Haven Conn. 305 Lawrence St.

Lonseth, Dr. A. T. Instr., Iowa State Coll., Ames, Iowa. Bureau of Ordnance, U. S. Navy Dept., Washington, D.C. 3027 Newark St.

Loomer, A. P. 7762 S. Foord St., Stellarton, N.S., Canada.

Loomis, Dr. L. H. Instr., Harvard Univ., Cambridge, Mass. 43 Museum St.

Lorch, E. R. Asst. Prof., Barnard Coll., Columbia Univ., New York, N.Y. 435 W. $123 \mathrm{~d}$ St.

Lorch, Dr. Lee. Asst. Mathematician, National Advisory Com. for Aeronautics, Langley Field, Hampton, Va. Box 293.

Lorell, Jack. 10 Liberty St., Aberdeen, Md.

§Lorenz, E. N..Fellow, Harvard Univ., Cambridge, Mass. 56 W. Hill Dr., West Hartford, Conn.

Lorenzen, Robert. 2156 Lexington Ave., New York, N.Y. Radio Engineer, Camp Murphy, Florida.

Loring, S. J. Research Engineer, Vought-Sikorsky Aircraft, Stratford, Conn. 63 Camden St., Devon, Conn.

de Losada y Puga, Christóbal. Prof., Catholic Univ. of Peru, Lima, Peru. Apartado 2708.

Lotka, Dr. A. J. Asst. Statistician, Metropolitan Life Ins. Co., 1 Madison Ave., New York, N.Y. Beattie Park, Red Bank, N.J.

$\S$ Loud, W. S. Massachusetts Inst. of Tech., Cambridge, Mass. 171 Commercial St., Weymouth, Mass.

Love, C. E. Prof., Univ. of Michigan, Ann Arbor, Mich. 1915 Scottwood.

$\dagger$ Lovett, E. O. Pres. Emeritus, Rice Inst., Houston, Tex.

Lowan, A. N. Asso. Prof., Yeshiva Coll., New York, N.Y.; Senior Mathematician, Mathematical Tables Project, National Bureau of Standards, U. S. Dept. of Commerce, 70 Columbus Ave., New York, N.Y. 312 Schenectady Ave., Brooklyn, N.Y.

Lowenstein, L. L. Asso. Prof., Alfred Univ., Alfred, N.Y.

Lubben, R. G. Asst. Prof., Univ. of Texas, Austin, Tex. W.H. 16, University Station.

Lubin, C. I. Asso. Prof., Univ. of Cincinnati, Cincinnati, Ohio. 3612 Washington Ave.

§Luckey, Dr. R. R. R. Instr., Houghton Co11., Houghton, N.Y.

$\S$ Lufkin, Dr. H. M. Instr., Univ. of Pennsylvania, Philadelphia, Pa. Bennett Hall.

Lukacs, Dr. Eugene. 5510 Pimlico Road, Baltimore, Md.

Luke, Y. L. Care of Alper, 1839 N. Whipple, Chicago, I11.

Luneburg, Dr. R. K. Research Dept., Spencer Lens Co., Buffalo, N.Y.

Lunn, A. C. Prof. Emeritus, Univ. of Chicago, Chicago, I11. 5211 Kenwood Ave.

Luther, C. F. Dean, Coll. of Liberal Arts, and Prof., Willamette Univ., Salem, Ore.

§Lyman, Theodore. Prof. Emeritus, Harvard Univ., Cambridge, Mass. Research Physics Lab.

Lynch, Rev. J. J. Prof., Fordham Univ., New York, N.Y.

§McCankie, R. C. Equitable Life Ins. Co. of Iowa, Des Moines, Iowa.

McCarthy, E. D. Asst. Prof., Coll. of Engineering, Univ. of Detroit, Detroit, Mich.

McCarthy, J. J. Asst. Prof., St. John's Univ., Brooklyn, N.Y. 211 Lafayette Ave.

McCarty, A. L. Prof., San Francisco Junior Coll., San Francisco, Calif. 1798 Grove St.

McCauley, C. E. Woodstock Coll., Woodstock, Md.

McClay, Dr. D. T. Instr., Univ. of Vermont and State Agric. Coll., Burlington, Vt. 37 Colonial Sq.

$\dagger$ McClenon, R. B. Prof., Grinnell Coll., Grinnell, Iowa. 1105 Park.

McConnell, A. J. Prof. of Philosophy, Univ. of Dublin, Dublin, Ireland. 36 Trinity Coll.

McConnell, H. J. Cartographic Engineer. 3328 Madison Ave., San Diego, Calif.

McCormick, J. T. Prof., Holy Cross Coll., Worcester, Mass. 
McCoy, Dorothy. Prof., Belhaven Coll., Jackson, Miss.

McCoy, N. H. Prof., Smith Coll., Northampton, Mass. 53 Ridgewood Ter.

McCrea, W. H. Prof., Queen's Univ., Belfast, Northern Ireland. Flying Officer, R.A.F.V.R.

$\S M c C u s k e y$, S. W. Asso. Prof., Case School of Applied Science, Cleveland, Ohio. McDaniel, R. R. Asso. Prof., Virginia State Coll., Ettrick, Va.

McDaniel, w. C. Asst. Prof., Southern Illinois State Normal Univ., Carbondale, I11.

McDermott, Eugene. Pres., Geophysical Service, Inc., 1311 Republic Bank, Da1las, Tex. Consultant, National Defense Research Com.

McDonough, Dr. D. L. 1205 Belfield Ave., Drexel Hill, Pa.

McDougle, Edith A. Instr., Women's Coll., Univ. of Delaware, Newark, Del.

McEwen, Dr. G. F. Scripps Inst., Univ. of California, La Jolla, Calif. Box 109.

McEwen, W. H. Prof., Mt. Allison Univ., Sackville, N.B., Canada.

McEwen, W. R. Instr., Univ. of Minnesota, Minneapolis, Minn.

McFadden, Dr. Leonard. Instr., Virginia Polytech. Inst., Blacksburg, Va. Box 65.

McFarlan, L. H. Asso. Prof., Univ. of Washington, Seattle, Wash. 158 Philosophy Hall.

McFarland, Dora. Asso. Prof., Univ. of Oklahoma, Norman, Okla. Faculty Exch.

McGaughey, Dr. A. W. Instr., U. S. Naval Acad., Annapolis, Md.

McGrath, Rev. P. H. Prof., St. Peter's Coll., 144 Grand St., Jersey City, N.J.

McKee, Dr. Ruth S. 3607 Brisban St., Harrisburg, Pa.

McKelvey, J. V. Prof., Iowa State Co11., Ames, Iowa. 2117 Graeber St.

McKelvey, Martha M. (Mrs. J. V.). Instr., Iowa State Coll., Ames, Iowa. 2117 Graeber St.

McKenney, Rev. J. L. Prof., Providence Coll., Providence, R.I.

McKinsey, Dr. J. C. C. Instr., Washington Square Coll., New York Univ., New York, N.Y. Guggenheim Fellow. Inst. for Advanced Study, Princeton, N.J.

McLaughlin, K. F. Junior Instr., U.S.A., Air Corps Tech. School, Scott Field, IIl. 307 E. Washington St., Belleville, I11.

McMahon, F. A. Brooklyn Coll., Brooklyn, N.Y. 40-18 Forley St., Elmhurst, N.Y.

McMaster, A. S. Asst. Prof., Engineering Math. Dept., Univ. of Colorado, Boulder, Colo.

McMillan, Dr. Audrey W. (Mrs. B.). Instr., Vassar Coll., Poughkeepsie, N.Y. Asst., Inst. for Advanced Study, Princeton, N.J.

McMillan, Dr. Brockway. Instr., Princeton Univ., Princeton, N.J.

McMullen, C. B. Prof., State Teachers Coll., Hyannis, Mass.

McNeil, Sister M. Gertrude. Prof., Seton Hill Coll., Greensburg, Pa.

McShane, E. J. Prof., Univ. of Virginia, University Station, Charlottesville, Va. 209 Maury Ave.

MacColl, Dr. L. A. Research Mathematician, Bell Telephone Labs., 463 West St., New York, N.Y.

MacCullough, R. H. Prof., Defiance Coll., Defiance, Ohio. Sisson Hall.

MacDonald, J. K. L. Asst. Prof., Cooper Union, New York, N.Y.

MacDougal, H. B. Prof., South Dakota State Coll., Brookings, S.Dak.

MacDuffee, C. C. Prof., Hunter Coll., 695 Park Ave., New York, N.Y.

§Mackey, Dr. G. W. Instr., Illinois Inst. of Tech., Chicago, Ill.

Mackie, E. L. Prof., Univ. of North Carolina, Chapel Hill, N.C. 702 Gimghoul Road.

§MacKinnon, W. D. Equitable Life Ins. Co. of Iowa, Des Moines, Iowa.

MacLane, G. R. Radiation Lab., Massachusetts Inst. of Tech., Cambridge, Mass. 7 Avon St.

MacLane, Saunders. Asso. Prof., Harvard Univ., Cambridge, Mass. 7 Avon St.

MacLean, N. B. Prof., McGill Univ., Montreal, Quebec, Canada.

MacNeille, H. M. Prof., Kenyon Coll., Gambier, Ohio.

MacNeish, H. F. Prof., Brooklyn Coll., Brooklyn, N.Y. 48 Taunton Road, Scarsdale, N.Y.

Macphail, M. S. Asst. Prof., Acadia Univ., Wolfville, N.S., Canada.

MacQueen, M. L. Prof., Southwestern Coli., Memphis, Tenn.

Maddaus, Ingo. Asst. Prof., Univ. of Oregon, Eugene, Ore.

Madden, Sister Teresa M. Prof., Coll. of Our Lady of the E1ms, Chicopee, Mass. 
Madow, Dr. W. G. Asso. Statistician Bureau of the Census, U. S. Dept. of Commerce, Washington, D. C. 1445 Ogden St. N.W.

$\S$ Maharam, Dr. Dorothy. Inst. for Advanced Study, Princeton, N.J.

Makarov, A. G. Instr., Univ. of Delaware, Newark, Del.

Maker, P. T. Asst. Prof., Duke Univ., Durham, N.C. 2803 Nation Ave.

Malin, Dr. Henry. Instr., Coll. of the City of New York, 139th St. and Convent Ave., New York, N.Y.

Mallory, V. S. Prof., New Jersey State Teachers Coll., Upper Montclair, N.J.

Maloney, Sister M. Michael. Instr., D'Youville Coll., 320 Porter Ave., Buffalo, N.Y.

Maloney, Sister Thomas M. Asso. Prof., Trinity Coll., Washington, D.C.

Malti, M. G. Asst. Prof., Cornell Univ., Ithaca, N.Y. Franklin Hall.

Manchester, R. E. Dean, Kent State Univ., Kent, Ohio.

Mancill, J. D. Asso. Prof., Univ. of Alabama, University, Ala.

Mandelbrojt, Szolem. Prof., Coll. of France, Paris, France; Visiting Prof., Rice Inst., Houston, Tex.

Mann, Dr. H. B. Research Asso., Economics Dept., Columbia Univ., New York, N.Y. 189 8th Ave., Brooklyn, N.Y.

$\$$ Mann, W. R. Asst., Univ. of California, Berkeley, Calif. American Friends Service Com., Paso de Ovejas, Veracruz, Mexico.

† Manning, H. P. Asso. Prof. Emeritus, Brown Univ., Providence, R.I. 148 Governor St.

Manning, Dr. Rhoda. Instr., Oregon State Coll., Corvallis, Ore. 321 N. 23d St.

Manning, W. A. Prof., Stanford Univ., Stanford University, Calif. 649 Alvarado Row.

Manring, W. E. Sales Engineer, American Cyanamid and Chemical Corp., 30 Rockefeller Plaza, New York, N.Y.

Mansfield, Ralph. Prof., Chicago Teachers Coll., Chicago, I11.

Many, Anna E. Prof., Newcomb Coll., Tulane Univ., New Orleans, La.

§Mapes, G. S. Asst., Univ. of Illinois, Urbana, I11. 160 Math. Bldg.

March, H. W. Prof., Univ. of Wisconsin; Head Mathematician, U. S. Forest Products Lab., Madison, Wis. 1825 Sumnit Ave.

Marcou, R. J. Prof., Boston Coll., Chestnut Hill, Mass.

Marden, Morris. Asso. Prof., Univ. of Wisconsin at Milwaukee, Milwaukee, Wis. 403 E. Carlisle Ave.

Maria, Dr. A. J. Instr., Brooklyn Coll., Brooklyn, N.Y.

Maria, Dr. Deborah H. (Mrs. A. J.). Brooklyn Coll., Brooklyn, N.Y.

Marm, Anna. Prof., Bethany Coll., Lindsborg, Kan. 741 N. 2d St.

Marquis, R. H. Prof., Ohio Univ., Athens, Ohio. Box 216.

Marriott, R. W. Prof., Swarthmore Coll., Swarthmore, Pa. 213 Lafayette Ave.

Marshall, William. Prof. Emeritus, Purdue Univ., Lafayette, Ind. R.F.D. 9.

Marston, Dr. A. E. Box 630, Monterey, Calif. Lt. (j.g.), U.S.N.R.

$\S$ Martin, Dr. F. L. Meteorologist, Northwest Airlines, Felts Field, Spokane, Wash.

Martin, M. H. Asso. Prof., Univ. of Maryland, College Park, Md.

Martin, Margaret $P$. Instr. in Biostatistics, DeLamar Inst. of Public Health, Columbia Univ., 600 W. 168th St., New York, N.Y.

Martin, W. T. Asst. Prof., Massachusetts Inst. of Tech., Cambridge, Mass.

Martyn, W. J. Otago Boys' High School, Dunedin, New Zealand.

\$Marx, Imanuel. New York Univ., New York, N.Y. 53 Washington Sq. S.

$\nmid$ Mason, Dr. Max. Chairman, Observ. Council, California Inst. of Tech., Pasadena, Calif.

Mason, W. E. Asso. Prof., Univ. of California at Los Angeles, Los Angeles, Calif.

Mason, Dr. W. P. Research Engineer, Bell Telephone Labs., 463 West St., New York, N.Y.

$\S$ Mathews, C. W. Asst., Univ. of Illinois, Urbana, I11. Marissa, I11. Ensign, U.S.N.R.

Mathewson, L. C. Prof., Dartmouth Coll., Hanover, N.H.

Matz, Joshua. Instr., Yeshiva Coll., New York, N.Y.

May, Dr. A. E. Instr., Univ. of Wisconsin at Kenosha, Kenosha, Wis. 416 68th St.

Mayer, Dr. Joanna I. 217 S. Moniteau, Sedalia, Mo. 
Mayer, Dr. Walther. Asso., Inst. for Advanced Study, Princeton, N.J.

Mayor, J. R. Prof., Southern Illinois Normal Univ., Carbondale, Ill.

Meacham, E. D. Dean, Coll. of Arts and Sciences, and Prof. Univ. of Oklahoma, Norman, Okla. Faculty Exch.

Mead, Sallie P. (Mrs. C. E.). Tech. Staff, Bell Telephone Labs., 463 West St., New York, N.Y.

Mears, Florence M. Asso. Prof., George Washington Univ., Washington, D.C.

Meder, A. E. Prof., New Jersey Coll. for Women, Rutgers Univ., New Brunswick, N.J.

Mehlenbacher, L. E. Prof., Arizona State Teachers Coll., Flagstaff, Ariz.

Melcher, Dr. George. Asst. Supt. of Schools, Kansas City, Mo. 201 W. 51st St.

Melchor, Alejandro. Prof. of Engineering, Philippine Military Acad., Baguio, Philippine Islands. Lt. Col., U.S.A., Engineer Board, Fort Belvoir, Va.

Mendel, Dr. C. W. Asso., Univ. of Illinois, Urbana, I11. 369 Math. Bldg.

$\S$ Mendelsohn, Dr. N. S. Supervisor, Munitions Gauge Lab., National Research Council, Ottawa, Ont., Canada.

Menger, Karl. Prof., Univ. of Notre Dame, Notre Dame, Ind. 1421 E. LaSalle St., South Bend, Ind.

Merrick, Rev. J. P. Prof., Baghdad Coll., Baghdad, Iraq.

Merrill, A. S. Prof., Montana State Univ., Missoula, Mont. Consultant, Civil Aeronautics Admin.

$\uparrow$ Merrill, Helen A. Prof. Emeritus, Wellesley Coll., Wellesley, Mass. 6 Waban St.

Merriman, G. M. Asso. Prof., Univ. of Cincinnati, Cincinnati, Ohio.

Mersman, Dr. W. A. Instr., Coll. of Agric., Univ. of California, Davis, Calif. Radiation Lab., Massachusetts Inst. of Tech., Cambridge, Mass. 395 Broadway.

$\S$ Meserve, B. E. Asst., Duke Univ., Durham, N.C. 1625 Forest Ave., Portland, Me. Civilian Public Service Camp, Buck Creek, Marion, N.C.

†Metzler, Dr. W. H. Retired. 172 High St., Lockport, N.Y.

Mewborn, A. B. Asst. Prof., Univ. of Arizona, Tucson, Ariz. 4425 University Station.

Meyer, Herman. Asst. Prof., Univ. of Miami, Coral Gables, Fla.

Meyer, H. A. Prof., Hanover Coll., Hanover, Ind.

Meyer, H. L. Instr., Coll. of Wooster, Wooster, Ohio.

Michal, A. D. Prof., California Inst. of Tech., Pasadena, Calif.

Michel, R. J. Prof., Southeast Missouri State Teachers Coll., Cape Girardeau, Mo. 225 N. Ellis St.

Mickelson, Dr. E. L. Fox Construction Co., Tucson, Ariz. 607 Bayard St., Silver City, N.Mex.

Midgley, Dr. Thomas. General Motors Chemical Co., Dayton, Ohio. R.F.D. 2, Worthington, Ohio.

Mijares, J. M. Prof., Mapua Inst. of Tech., Manila, Philippine Islands.

Miles, E. J. Asso. Prof., Yale Univ., New Haven, Conn. 87 Marvel Road.

Miles, E. P. 837 7th St. W., Birmingham, Ala. Ensign, U.S.N.R., Supply Dept., U. S. Naval Station, New Orleans, La.

Miles, Dr. H. J. Asso., Univ. of Illinois, Urbana, I11. 253 Math. Bldg.

Milgram, A. N. Asst. Prof., Univ. of Notre Dame, Notre Dame, Ind. 1006 E. Ewing St., South Bend, Ind.

Miller, Dr. A. L. Treas., C. N. Miller Co., Boston, Mass. 25 Clinton Road, Brookline, Mass.

Miller, C. E. Asst. Prof., Univ. of New Brunswick, Fredericton, N.B., Canada.

Miller, Dr. D. D. Instr., Ohio Univ., Athens, Ohio. Faculty Club. Lt. (j.g.), U.S.N.R.

Miller, Dr. D. S. Instr., Yale Univ., New Haven, Conn.

Miller, E. A. 113 N. Tioga St., Ithaca, N.Y.

Miller, F. A. Instr., Wentworth Military Acad., Lexington, Mo. Capt., U.S.A.

Miller, F. H. Asst. Prof., Cooper Union, New York, N.Y.

†Miller, G. A. Prof., Univ. of Illinois, Úrbana, I1l. 1203 W. Illinois St.

Miller, Harlan C. Asso. Prof., North Texas State Teachers Coll., Denton, Tex.

Miller, H. L. Asso. Prof., Univ. of Cincinnati, Cincinnati, Ohio.

Miller, Dr. J. A. Wallingford, $\mathrm{Pa}$. 
Miller, J. L. 542 Georgia Ave., Brooklyn, N.Y. Junior Inspector, Office of Inspectors of Naval Materiel, U. S. Navy Dept. 30 Church St., New York, N.Y.

Miller, Norman. Prof., Queen's Univ., Kingston, Ont., Canada.

Miller, O. M. Mathematical Geography Dept., American Geographical Society, Broadway and 156th St., New York, N.Y.

Miller, R. A. Lt., A.U.S., Pre-Flight School, Maxwell Field, Ala.

Miller, W. I. Asst. Prof., Bucknell Univ., Lewisburg, Pa. 220 S. 3d St.

§Mills, M. M. Research Asst. in Aeronautics, California Inst. of Tech., Pasadena, Calif. 405 Northcliffe Road.

$\S$ Millsaps, K. T. California Inst. of Tech., Pasadena, Calif.

Milne, W. E. Prof., Oregon State Coll., Corvallis, Ore.

Milne, W. P. Prof., Univ. of Leeds, Leeds, England. 16 Monkbridge Road, Headingly.

Mintzer, H. L. Fellow, Brown Univ., Providence, R.I.

Mira, J. A. Prof. Manhattanville Coll. of the Sacred Heart, New York, N.Y. 61 E. 86th St.

Mirick, G. R. Prin., High School, Lincoln School, 425 W. 123d St., New York, N.Y.

Miser, H. J. Instr., Illinois Inst., of Tech., Chicago, Ill.

Miser, W. L. Prof., Vanderbilt Univ., Nashville, Tenn. 1702 Cedar Lane.

von Mises, Dr. R. E. Lecturer, Harvard Univ., Cambridge, Mass.

Mitchell, A. K. Asst. Prof., Trinity Coll., Hartford, Conn.

Mitchell, H. B. Chairman of the Board, Griscom-Russell Co., 285 Madison Ave., New York, N.Y. Chapel Farm, Riverdale-on-Hudson, N.Y.

Mitchell, H. H. Prof., Univ. of Pennsylvania, Philadelphia, Pa. College Hall.

$\S$ Mitchell, Dr. Josephine M. Instr., Hollins Coll., Hollins College, Va.

Mitchell, Dr. W. L. Instr., Michigan State Coll., East Lansing, Mich. 317 Evergreen Ave.

§Mittleman, Don. 300 Fort Washington Ave., New York, N.Y.

Mode, E. B. Prof., Boston Univ., Boston, Mass. Longmeadow Road, Wellesley, Mass.

Modesitt, Dr. Virginia. Wright Junior Coll., Chicago, I11.

Molina, E. C. Switching Theory Engineer, Bell Telephone Labs., 463 West St., New York, N.Y.

Monsky, Morris. Asst. Actuary, Mutual Life Ins. Co. of N.Y., 32 Nassau St., New York, N.Y.

Montague, Harriet F. Asst. Prof., Univ. of Buffalo, Buffalo, N.Y.

Montgomery, A. G. Asst., Univ. of Minnesota, Minneapolis, Minn. 204 Main Engineering Bldg.

Montgomery, Deane. Prof., Smith Coll., Northampton, Mass.

Montgomery, Dr. J. C. Instr., Univ. of Connecticut, Storrs, Conn.

Montgomery, P. v. Vice Pres. and Actuary, Southland Life Ins. Co., Dallas, Tex.

Montroll, Dr. E. W. Instr., Princeton Univ., Princeton, N.J. Palmer Physics Lab.

Moody, W. A. Prof. Emeritus, Bowdoin Coll., Brunswick, Me. 60 Federal St.

Moon, Parry. Asso. Prof., Massachusetts Inst. of Tech., Cambridge, Mass.

$\$$ Moore, B. C. Asst. Prof., Agric. and Mech. Coll. of Texas, College Station, Tex. Box 424, Laguna Beach, Calif. Lt., U.S.N.R.

Moore, C. N. Dir. of Graduate Studies, and Prof., Univ. of Cincinnati, Cincinnati, Ohio. 219 Woolper Ave.

Moore, Dr. Lillian. Far Rockaway High School, Far Rockaway, N.Y.; General Asst., Central Evening High School, Brooklyn, N.Y. 885 N. 28th St., Philadelphia, $\mathrm{Pa}$.

Moore, L. T. Asso. Prof., Brooklyn Coll., Brooklyn, N.Y. 205 Hicks St.

Moore, M. G. Prof., Tri-State Coll., Angola, Ind. 301 E. Gilmore.

† Moore, R. L. Prof., Univ. of Texas, Austin, Tex.

Moore, T. W. Asst. Prof., U. S. Naval Acad., Annapolis, Md.

Moore, w. L. Asso. Prof., Univ. of Louisville, Louisville, Ky. Box 163, R.F.D. 1, Coral Ridge, Ky.

Moots, E. E. Prof., Cornell Coll., Mt. Vernon, Iowa.

Morales, J. D. Prof., Univ. of Puerto Rico, Mayaguez, Puerto Rico. Box 882.

Moran, Dr. C. W. Lane Tech. School, Chicago, I11. 2950 Jarlath St.

Mordell, L. J. Prof., Univ. of Manchester, Manchester, England. 
Morehead, J. C. Prof. Emeritus, Carnegie Inst. of Tech., Pittsburgh, Pa. 4815 Bayard St.

Morenus, Eugenie M. Prof., Sweet Briar Coll., Sweet Briar, Va.

Morgan, Dr. F. M. Dir., Clark School, Hanover, N.H.

Morgan, W. B. 6728 Woodland Ave., Philadelphia, Pa. Civilian Public Service Camp, Fresno, Ohio.

Morkovin, Dr. Vladimir. Fellow, School of Mech., Brown Univ., Providence, R.I.

Morley, R. K. Prof., Worcester Polytech. Inst., Worcester, Mass.

Morrey, C. B. Asso. Prof., Univ. of California, Berkeley, Calif. Mathematician, Ballistic Research Lab., Aberdeen Proving Ground, Md.

Morrill, Dr. W. K. Asso., Johns Hopkins Univ., Baltimore, Md. 534 Stevenson Lane, Towson, Md.

Morris, F. R. Prof., Fresno State Coll., Fresno, Calif.

Morris, Max. Asso. Prof., Case School of Applied Science, Cleveland, Ohio.

Morris, Richard. Prof., Rutgers Univ., New Brunswick, N.J. 12 Johnson St.

Morris, Dr. W. L. Mathematician, Research Dept., Phillips Petroleum Co., Bartlesville, Okla. 519 Keeler St.

Morrison, Nathan. Senior Statistician, Div. of Research and Statistics, U. S. Employment Service, 342 Madison Ave., New York, N.Y. 420 Ave. F, Brooklyn, N.Y.

Morrow, D. C. Asso. Prof., Wayne Univ., Detroit, Mich.

§Morrow, Dorothy J. Fellow, Bryn Mawr Coll., Bryn Mawr, Pa. 4332 8th N.E., Seattle, Wash.

Morse, A. P. Asst. Prof., Univ. of California, Berkeley, Calif. 18 Oak Vale.

Morse, D. S. Prof., Union Coll., Schenectady, N.Y.

Morse, Marston. Prof., Inst. for Advanced Study, Princeton, N.J.

Mosesson, Dr. Z. I. Prudential Ins. Co. of America, Newark, N.J. U.S.A., Box 192, Ft. Monroe, Va.

Moskovitz, David. Asst. Prof., Carnegie Inst. of Tech., Pittsburgh, Pa.

Mossman, Thirza A. Asst. Prof., Kansas State Coll. of Agric. and Applied Science, Manhattan, Kan.

Moulton, E. J. Prof., Northwestern Univ., Evanston, I11. 1114 Colfax St.

Moulton, Dr. F. R. Permanent Sec., American Asso. for the Advancement of Science, Smithsonian Inst. Bldg., Washington, D.C.

Moursund, A. F. Asso. Prof., Univ. of Oregon, Eugene, Ore.

Mowbray, A. H. Prof., Univ. of California, Berkeley, Calif. 119 South Hall.

Muhly, Dr. H. T. Instr., U. S. Naval Acad., Annapolis, Md. 5 School St.

Muhly, Isabel F. (Mrs. H. T.). 5 School St., Annapolis, Md.

Mullemeister, Hermance. Asst. Prof., Univ. of Washington, Seattle, Wash. 1900 E. 68th St.

Mullings, M. E. Prof., Abilene Christian Coll., Abilene, Tex.

Mullins, G. W. Prof., Barnard Coll., Columbia Univ., New York, N.Y.

Mumford, C. G. Asso. Prof., North Carolina State Coll., Raleigh, N.C. State College Station.

Munro, G. C. Asso. Prof., Kansas State Coll. of Agric. and Applied Science, Manhattan, Kan.

$\S$ Munro, W. D. Asst., Univ. of Minnesota, Minneapolis, Minn. 123 Folwell Hall.

Munroe, Florence L. Retired. 51 Henshaw Ave., Northampton, Mass.

\$Munroe, M. E., Fellow, Brown Univ., Providence, R.I.

Munshower, C. W. Asso. Prof., Colgate Univ., Hamilton, N.Y.

Murdoch, D. C. Asst. Prof., Univ. of Saskatchewan, Saskatoon, Sask., Canada.

Murnaghan, F. D. Prof., Johns Hopkins Univ., Baltimore, Md. 6202 Sycamore Road.

Murphy, R. D. Vice Pres. and Actuary, Equitable Life Assurance Society of the U.S., 120 Broadway, New York, N.Y. 28 Godfrey Road, Upper Montclair, N.J.'

Murray, Dr. F. H. 5412 Kimbark Ave., Chicago, I11.

Murray, F. J. Asst. Prof., Columbia Univ., New York, N.Y. 2 Arden St.

Murray, w. R. Asso. Prof., Franklin and Marshall Coll., Lancaster, Pa.

Murtaugh, T. H. Asst. Registrar, School of Education, Fordham Univ., New York, N.Y. 597 North Ave., New Rochelle, N.Y. 
Musselman, J. R. Prof., Western Reserve Univ., Cleveland, Ohio.

Myers, F. G. Instr., Univ. of Virginia, University Station, Charlottesville, Va. 1410 Park P1.

Myers, S. B. Asst. Prof., Univ. of Michigan, Ann Arbor, Mich. 3020 Angell Hall.

Myers, W. H. Asst. Prof., San Jose State Coll., San Jose, Calif. 1344 Heddings St. $2 d$ Lt., U.S.A., Ordnance Presidio, San Francisco, Calif.

Nagel, Ernest. Asso. Prof., Philosophy Dept., Columbia Univ., New York, N.Y.

Nagle, J. L. Head Engineer, Caribbean Div., U.S.A., Corp of Engineers, 711 Park Ave., Elizabeth, N.J.

Nahikian, Dr. H. M. Instr., North Carolina State Coll., Raleigh, N.C. 5548 State College Station.

$\S N$ aim, Yael. Johns Hopkins Univ., Baltimore, Md.

Nakayama, Tadasi. Asst. Prof., Osaka Imperial Univ., Osaka, Japan.

Nardelli, M. J. Asst. Prof., Fordham Univ., New York, N.Y.

Nash, Dr. J. P. Instr., Univ. of Notre Dame, Notre Dame, Ind. Research Asso., Radiation Lab., Massachusetts Inst. of Tech., Cambridge, Mass. 541 Commonwealth Ave., Boston, Mass.

Neelley, J. H. Prof., Carnegie Inst. of Tech., Pittsburgh, Pa. 300 Broadmoor Ave., Mt. Lebanon, Pa.

Neikirk, L. I. Asst. Prof., Univ. of Washington, Seattle, Wash. 4723 21st Ave. N.E.

Nelson, A. L. Prof., Wayne Univ., Detroit, Mich.

Nelson, C. A. Asso. Prof., New Jersey Coll. for Women, Rutgers Univ., New Brunswick, N.J.

Nelson, J. B. Instr., Evening Div., Univ. of Southern California, Los Angeles, Calif. 6345 Ben Ave., North Hollywood, Calif.

$\S N$ elson, M. L. Asst., Univ. of California, Berkeley, Calif.

Nelson, N. D. Asst. Univ. of Wisconsin, Madison, Wis.

Nelson, Sara L. Prof., Georgia State Coll. for Women, Milledgeville, Ga. 512 N. Columbia St.

Nesbitt, C. J. Asst. Prof., Univ. of Michigan, Ann Arbor, Mich. 3016 Angell Hall.

Ness, Marie M. Research Asso., Univ. of Minnesota, Minneapolis, Minn. 2530 Dupont Ave. S.

Netzorg, Dr. D. L. Box 2714, Manila, Philippine Islands.

Neugebauer, Otto. Prof., Brown Univ., Providence, R.I.

Neuhaus, Dr. Albert. Instr., Univ. of Nebraska, Lincoln, Neb. 1718 N St.

Neuman, Sidney. 717 W. 177th St., New York, N.Y.

von Neumann, John. Prof., Inst., for Advanced Study, Princeton, N.J. Bureau of Ordnance, U. S. Navy Dept., Washington, D.C.

Newell, Dr. H. E. Instr., Univ. of Maryland, College Park, Md.

Newman, J. R. 6019 N. 19th Road, Arlington, Va.

Newman, Dr. Philip. Coll. of the City of New York, New York, N.Y.

Newsom, C. V. Prof., Univ. of New Mexico, Albuquerque, N.Mex. 105 S. 14th St.

Newson, Dr. Mary W. (Mrs. H. B.). Retired. Eureka Coll., Eureka, I11.

Newton, Abba V. Prof., Hartwick Coll., Oneonta, N.Y. 22 Maple St.

Newton, G. A. Prof., Trinity Univ., San Antonio, Tex.

Neyman, Jerzy. Dir., Statistical Lab., and Prof., Univ. of California, Berkeley, Calif. National Defense Research Com.

Nichols, G. D. Asso. Prof., Univ. of Arkansas, Fayetteville, Ark.

Nielsen, Dr. K. L. Instr., Louisiana State Univ., University, La.

Nightwine, Sister M. Cyprian. Asst. Prof., Xavier Univ., New Orleans, La.

Nilson, E. N. Asst. Prof., Mt. Holyoke Coll., South Hadley, Mass. 31 Woodbridge St.

Niven, Dr. Ivan. Instr., Purdue Univ., Lafayette, Ind.

Noble, Dr. Andrewa R. Instr., San Francisco Junior Coll., San Francisco, Calif. 14957 th Ave.

Noble, C. A. Prof. Emeritus, Univ. of California, Berkeley, Calif. 2224 Piedmont Ave.

Noble, C. E. Northeast Missouri State Teachers Coll., Kirksville, Mo. 407 S. Main St.

Nodelman, H. M. Instr., Radio Corp. of America Inst., Inc., 75 Varick St., New York, N.Y. 
Noether, G. E. U.S.A., Antiaircraft Artillery School, Camp Davis, N.C.

Nordhaus, Dr. E. A. Instr., Univ. of Wisconsin at Milwaukee, Milwaukee, Wis.

Nordling, Dr. C. G. A. Instr., Univ. of Connecticut, Storrs, Conn.

Norlie, O. M. Prof., St. Olaf Coll., Northfield, Minn.

Nörlund, N. E. Prof., Univ. of Copenhagen, Copenhagen, Denmark. Stockholmsgade 33 .

Norman, P. B. Instr., Univ. of Wisconsin, Madison, Wis.

$\S$ Norris, M. J. Fellow, Harvard Univ., Cambridge, Mass. 3544 Portland Ave. S., Minneapolis, Minn.

Norris, Dr. Nilan. Instr., Economics Dept., Hunter Coll., 695 Park Ave., New York, N.Y.

Norris, R. E. Prof., State Teachers Coll., Milwaukee, Wis.

$\S$ Northham, J. I. Instr., U.S.A., Signal Corps, Chicago, I1l. 828 W. Kalamazoo Ave., Kalamazoo, Mich.

Northcott, J. A. Asso. Prof., Columbia Univ., New York, N.Y.

Northrop, Dr. E. P. Hotchkiss School, Lakeville, Conn.

Norton, K. A. Senior Radio Engineer, Federal Communications Com., Washington, D.C. 4623 Kenmore Dr. N.W.

Nyswander, J. A. Asso. Prof., Univ. of Michigan, Ann Arbor, Mich.

Oakley, C. O. Prof., Haverford Coll., Haverford, Pa.

Oberbeck, T. E. 7230 Glades Ave., Richmond Heights, Mo. Ensign, U.S.N.R.

Oberg, Dr. E. N. Instr., State Univ. of Iowa, Iowa City, Iowa.

Oboukhoff, N. M. Prof., Oklahoma Agric. and Mech. Coll., Stillwater, Okla. 402 Jefferson St.

O'Brien, Sister Catherine M. Prof., Coll. of Mt. St. Vincent, New York, N.Y.

O'Callahan, Rev. J. T. Holy Cross Coll., Worcester, Mass.

O'Connor, Rev. R. E. Prof., Loyola Coll., Montreal, Quebec, Canada.

Odle, Dr. J. W. Research Mathematician, U. S. Rubber Co., Detroit, Mich. 20506 Picadilly.

O'Donnell, G. A. Dean, Graduate School, Boston Coll., Chestnut Hill, Mass.

Ogden, E. B. Prof., Union Coll., Lincoln, Neb. 4626 Bancroft Ave.

Ogg, F. C. Prof., Bowling Green State Univ., Bowling Green, Ohio.

Oglesby, E. J. Prof., Univ. of Virginia, University Station, Charlottesville, Va. Box 1032 .

Oldenburger, Rufus. Prof., Illinois Inst. of Tech. Chicago, I11. Math. Consultant, Woodward Governor Co. and Askania Regulator Co.

Olds, C. D. Instr., Purdue Univ., Lafayette, Ind. 208 W. Stadium, West Lafayette, Ind.

Olds, E. G. Asso. Prof., Carnegie Inst. of Tech., Pittsburgh, Pa. 953 La Clair Ave., Regent Sq.

Ollivier, Arthur. Asst. Prof., Mississippi State Coll., State College, Miss. Box 405 .

Ollmann, L. F. Asso. Prof., Hofstra Coll., Hempstead, N.Y.

Olmstead, Dr. P. S. Lecturer, Stevens Inst. of Tech., Hoboken, N.J.; Tech. Staff, Bell Telephone Labs., 463 West St., New York, N.Y. Box 72, Essex Fells, N.J.

Olmsted, Dr. J. M. H. Instr., Univ. of Minnesota, Minneapolis, Minn. 111 Arthur Ave. S.E.

Olshen, Dr. A. C. Actuary and Chief Examiner, Oregon Ins. Dept., State Office Bldg., Salem, Ore.

Olson, Emma J. Asst. Prof., Kent State Univ., Kent, Ohio. 537 S. Lincoln St.

Olson, F. C. W. Physicist, Research Dept., American Can Co., Maywood, Ill.

Olson, H. L. Asst. Prof., Southwestern Univ., Georgetown, Tex. 1017 College St.

O'Neill, Sister Anna C. Dean, Coll. of St. Elizabeth, Convent Station, N.J.

Opatowski, Dr. Isaac. Armour Research Foundation, Illinois Inst. of Tech., Chicago, Ill.

Oppenheim, Alexander. Prof., Raffles Coll., Singapore, Straits Settlements.

Ore, Oystein. Prof., Yale Univ., New Haven, Conn. 326 Hall of Graduate Studies.

Oseen, C. W. Prof., Nobel Inst., Stockholm, 50, Sweden.

Osgood, W. F. Prof. Emeritus, Harvard Univ., Cambridge, Mass. 10 Dorset Road, Belmont, Mass. 
Ott, E. R. Asso. Prof., Univ. of Buffalo, Buffalo, N.Y. 20 Nicholson Ave.

Ott, W. P. Prof., Univ. of Alabama, University, Ala.

Overman, J. R. Dean, Coll. of Liberal Arts, and Prof., Bowling Green State Univ., Bowling Green, Ohio.

Owens, F. W. Prof., Pennsylvania State Coll., State College, Pa.

§Owens, Dr. Glynn. Massachusetts Inst. of Tech., Cambridge, Mass.

Owens, Dr. Helen B. (Mrs. F. W.). 462 E. Foster Ave., State College, Pa.

Oxtoby, J. C. Asst. Prof., Bryn Mawr Coll., Bryn Mawr, Pa.

Page, Leigh. Prof., Physics Dept., Yale Univ., New Haven, Conn.

Paige, L. J. 686 W. Irving Park Rd., Chicago, Ill. Ensign, U.S.N.R.

Pall, Gordon. Asst. Prof., McGill Univ., Montreal, Quebec, Canada. Engineering Bldg.

Palm, W. G. No address. 1st Lt., U.S.A.

Palmer, T. P. Instr., Rose Polytech. Inst., Terre Haute, Ind. 592 Lincoln Ave., St. Paul, Minn. $2 d$ Lt., U.S.A.

Palmquist, Dr. K. L. Instr., Southern Methodist Univ., Dallas, Tex. Asso. Ground School Instr., U. S. Naval Air Station, Cabaniss Field, Corpus Christi, Tex. Box 3031 .

§Panofsky, Dr. W. K. H. Asso. Physicist, National Defense Research Com., California Inst. of Tech., Pasadena, Calif.

$\dagger$ Paranjpye, Sir R. P. Retired. Purushattam Ashram, Poona, 4, India.

Park, Eugene. No address.

Park, H. V. Asst. Prof., North Carolina State Coll., Raleigh, N.C. 5548 State College Station.

Park, R. S. Prof., Eastern Kentucky State Teachers Coll., Richmond, Ky. 213 Burnam Ct.

Parke, N. G. Aeronautical Engineer, Bureau of Aeronautics, U.S. Navy Dept., Washington, D.C. Malvern Ave., Ruxton, Md.

Parker, S. T. Instr., Univ. of Louisville, Louisville, Ky. 2626 Pindell Ave.

Parker, W. v. Prof., Louisiana State Univ., University, La.

Parsons, C. H. 415 W. 115th St., New York, N.Y.

Pate, Dr. R. S. Instr., Univ. of Kansas, Lawrence, Kan.

Patten, W. E. Asst. Hydraulic Engineer, U.S. Dept. of Agric. 1306 Irish St., South Boston, Va.

Patterson, B. C. Prof., Hamilton Coll., Clinton, N.Y.

Patterson, G. W. Asst. Physicist, National Hydraulic Lab., National Bureau of Standards, U.S. Dept. of Commerce, Washington, D.C.

Patterson, Dr. J. O. Retired. 16 W. Lynnwood Ave., Glenside, Pa.

Patterson, W. A. Asst. Prof., Fenn Coll., Cleveland, Ohio. 16601 Raymond St., Maple Heights, Ohio.

Paxson, Dr. E. W. Instr., Wayne Univ., Detroit, Mich. Mathematician, Jam Handy Organization, Detroit, Mich.

Paxton, E. K. Asso. Prof., Washington and Lee Univ., Lexington, Va. Box 754.

Paydon, J. F. Instr., Northwestern Univ., Evanston, Ill.

Payne, C. K. Asst. Prof., Washington Square Coll., New York Univ., New York, N.Y. Butler, N.J.

§Peabody, Mary K. Yale Univ., New Haven, Conn. 246 Park St.

Peach, M. O. Instr. of Mech., Carnegie Inst. of Tech., Pittsburgh, Pa. 51 Maytide St.

Pease, E. M. J. Asst. Prof., Rhode Island State Coll., Kingston, R.I.

Peck, E. R. Research, National Defense Research Com., Physics Dept., Northwestern Univ., Evanston, I11. 1233 Crain St.

$\S$ Peck, L. G. Johns Hopkins Univ., Baltimore, Md.

Peebles, G. H. Asst. Prof., Univ. of Minnesota, Minneapolis, Minn. 207 Main Engineering B1dg.

Pegram, Annie M. Prof., Greensboro Coll., Greensboro, N.C.

Pehrson, E. W. Prof., Univ. of Utah, Salt Lake City, Utah.

Pell-Wheeler, Anna. See Wheeler, Anna Pell.

Pence, Sallie E. Asst. Prof., Univ. of Kentucky, Lexington, Ky. 635 Maxwelton Ct. 
Penney, W. F. U. S. Navy Dept., Washington, D.C. 621 19th St. N.W.

Pepper. Dr. Echo D. Counselor, Personnel Bureau, and Asso., Univ. of Illinois, Urbana, I11. 356 Math. Bldg.

Pepper, P. N. Asst. Prof., Univ. of Notre Dame, Notre Dame, Ind.

Perez, Francisco. Asso. Prof., Univ. of the Philippines, Manila, Philippine Islands.

Perkins, F. W. Prof., Dartmouth Coll., Hanover, N.H. 8 Prospect St.

Perkins, H. A. Asso. Prof., Hampton Inst., Hampton, Va.

Perlin, Dr. I. E. Instr., Illinois Inst. of Tech., Chicago, I11.

§Perlis, Dr. Sam. Lockheed Aircraft Corp., Burbank, Calif. 140 N. Jackson St., Glendale, Calif.

Peters, Dr. A. S. Instr., New York Univ., University Heights, New York, N.Y.

Peters, Dr. J. W. Instr., Univ. of Illinois, Urbana, Ill. 360 Math. Bldg.

Peters, Ruth M. Prof., Lake Erie Coll., Painesville, Ohio.

Peterson, O. J. Prof., Kansas State Teachers Coll., Emporia, Kan.

Peterson, T. S. Asst. Prof., Univ. of Oregon, Eugene, Ore. Physicist, Degaussing Section, 13th Naval District, Seattle, Wash. 2408 N. 45th St.

Petrie, G. W. Lt. (j.g.), U.S.N.R., Midshipmen's School, New' York, N.Y. 419 W. 119th St.

Pettis, Dr. B. J. Box 673, Spartanburg, S.C. $2 d$ Lt., U.S.A., Army Post Office, San Francisco, Calif.

Pettis, C. S. Prof. of Physics, Wofford Coll., Spartanburg, S.C. Box 673.

Pettit, H. P. Prof., Marquette Univ., Milwaukee, Wis. Waterford, Wis.

Pfeiffer, G. A. Asso. Prof., Columbia Univ., New York, N.Y. 1110 Anderson Ave., Palisade, N.J.

Phalen, H. R. Asso. Prof., Coll. of William and Mary, Williamsburg, Va. Box 493.

Phelps, Dr. C. R. Ensign, U.S.N.R., U. S. Naval Acad., Annapolis, Md.

Philip, Maximilian. Prof., Coll. of the City of New York, 17 Lexington Ave., New York, N.Y.

Phillips, H. B. Prof., Massachusetts Inst. of Tech., Cambridge, Mass.

Phillips, Dr. R. S. Instr., Harvard Univ., Cambridge, Mass. 20 Highland Ave.

Phipps, C. G. Asso. Prof., Univ. of Florida, Gainesville, Fla. 2132 University Station.

Pierce, J. A. Prof., Atlanta Univ., Atlanta, Ga.

Pierce, T. A. Prof., Univ. o $x^{\circ}$ braska, Lincoln, Neb. 1811 Pepper Ave.

Pietenpol, H. W. Dean a1 Prof., Central Coll., Pella, Iowa.

§Pinney, Dr. Edmund. F ......h Asso., Radiation Lab., Massachusetts Inst. of Tech., Cambridge, Mass.

Pipes, Dr. L. A. Instr. in Electrical Engineering, Harvard Univ., Cambridge, Mass.

Piranian, George. Fellow, Rice Inst., Houston, Tex.

Pitcher, (A.) E. Asst. Prof., Lehigh Univ., Bethlehem, Pa. 422 W. Broad St. $2 d$ Lt., A.U.S., Aberdeen Proving Ground, Md.

Pixley, Emily C. (Mrs. H. H.). Prof., St. Francis Xavier Coll. for Women, 4900 Cottage Grove Ave., Chicago, I11. 20194 Briarcliff Ave., Detroit, Mich.

Pixley, H. H. Asso. Prof., Wayne Univ., Detroit, Mich

Plancherel, Michel. Prof., Federal Inst. of Tech., Zurich, Switzerland. Zeltweg 66.

Plant, L. C. Prof., Michigan State Coll., East Lansing, Mich

Podmele, Theresa L. East High School, Buffalo, N.Y. 356 Lisbon Ave.

Polachek, Harry. Ballistic Research Lab., Aberdeen Proving Ground, Md. 25 Swan St., Aberdeen, Md.

Polansky, F. J. Actuarial Dept., Northwestern National Life Ins. Co., Minneapolis, Minn. 767 Portland Ave., St. Paul, Minn.

Polanyi, M. Helen (Mrs.). Bennington Coll., Bennington, Vt.

Pollard, Dr. Harry. Instr., Harvard Univ., Cambridge Mass. 58 Waldeck St., Dorchester, Mass.

Pollard, H. S. Asso. Prof., Miami Univ., Oxford, Ohio. 350 Patterson Ave.

Polley, J. C. Prof., Wabash Coll., Crawfordsville, Ind.

Polya, George. Asso. Prof., Stanford Univ., Stanford University, Calif.

§Pond, J. A. Asst., Lehigh Univ., Bethlehem, $\mathrm{Pa}$.

Poor, C. L. Prof., Columbia Univ., New York, N.Y. 35 E. 69th St. 
Poor, V. C. Asso. Prof., Univ. of Michigan, Ann Arbor, Mich. 930 Packard St.

Poritsky, Dr. Hillel. General Electric Co., Schenectady, N.Y. 1012 Wendell Ave.

Porter, E. A. Sec. and Actuary, Manhattan Life Ins. Co., New York, N.Y. 17 Glen Ridge Pkwy., Bloomfield, N.J.

Porter, Goldie Horton (Mrs. M. B.). Adj. Prof., Univ. of Texas, Austin, Tex. 2402 Windsor Road.

Porter, J. W. Asso. Prof., Bluefield State Teachers Coll., Bluefield, W.Va. Asst. Ground School Instr., U.S.N., Chicago Vocational School, Chicago, Ill. 528 E. 34th St.

Porter, M. B. Prof., Univ. of Texas, Austin, Tex. 2402 Windsor Road.

Porter, Ruth E. Albany Senior High School, Albany, Ore. Ellsworth Apts.

Post, E. L. Asst. Prof., Coll. of the City of New York, New York, N.Y. 610 W. $173 \mathrm{~d}$ St.

Potron, M. M. J. Prof., Catholic Inst., Paris, France. 42 rue de Grenelle, Paris, 7, France.

Pound,' V. E. Prof., Univ. of Buffalo, Buffalo, N.Y. Hayes Hall.

Pounder, I. R. Prof., Univ. of Toronto, Toronto, Ont., Canada.

Povey, Kenneth. Lib., Queen's Univ., Belfast, Northern Ireland.

Powell, J. E. Asso. Prof., Michigan State Coll., East Lansing, Mich.

†Powers, R. E. Box 511, Denver, Colo.

§Prager, Willy. Prof. of Applied Mech., Brown Univ., Providence, R.I.

Pratt, E. A. Civil Engineer, Barber, N.J. R.F.D. 1, Scotch Plains, N.J.

Preiser, T. H. Financial Advisor. 10 Haim Cohen St., Petah Tikvah, Palestine.

Prenowitz, Walter. Asst. Prof., Brooklyn Coll., Brooklyn, N.Y. 2610 Glenwood Road.

Preston, Bernard. Certified Public Accountant, 103 Park Ave., New York, N.Y.

Pretz, P. S. Prof., St. Benedict's Coll., Atchison, Kan.

Price, G. B. Asso. Prof., Univ. of Kansas, Lawrence, Kan. 209 Frank Strong Hall.

Price, Dr. H. V. Instr., University High School and State Univ. of Iowa, Iowa City, Iowa.

Price, Irene. Prof., State Teachers Coll., Oshkosh, Wis.

§Price, J. F. Asst., Univ. of Illinois, Urbana, I11. 303 2d St. S., Forest Grove, Ore. U.S.A. Aberdeen Proving Ground, Md.

Protter, M. H. Asst., Brown Univ., Providence, R.I. 121 Power St.

Puckett, W. T. Asst. Prof., Univ. of California at Los Angeles, Los Angeles, Calif.

Pulliam, F. M. Prof., Lambuth Coll., Jackson, Tenn. 1420 N. Main St., Decatur, I11. U.S.M.C.R.

Purcell, E. J. Asso. Prof., Univ. of Arizona, Tucson, Ariz. Box 738, R.F.D. 5.

§ Putnam, Dr. A. L. Instr., Yale Univ., New Haven, Conn. 781 Yale Station.

$\S$ Putnam, George. 50 State St., Boston, Mass.

Putnam, R. G. Asso. Prof., Washington Square Coll., New York Univ., New York, N.Y. 115 Riverview Ave., Tarrytown, N.Y.

Putnam, T. M. Dean, Univ. of California, Berkeley, Calif.

Quade, E. S. Asst. Prof., Univ. of Florida, Gainesville, Fla.

Querry, J. W. Prof., Sam Houston State Teachers Coll., Huntsville, Tex. 1313 21 st St.

Quilliam, V. V. Cost Accountant, Firestone Tire and Rubber Co., 2525 E. Firestone Blvd., Los Angeles, Calif. 3771 May St., Venice, Calif.

Quinn, Dr. Grace S. (Mrs. R. B.). U. S. Navy Dept., Washington, D.C. 3221 Wheeler Road S.E.

Rademacher, Hans. Prof., Univ. of Pennsylvania, Philadelphia, Pa. Box 247, Swarthmore, $\mathrm{Pa}$.

Rado, Tibor. Prof., Ohio State Univ., Columbus, Ohio. 92 Walhalla Road.

Rafferty, T. C. Consulting Actuary, 233 International Bldg., St. Louis, Mo.

Rahn, Edris P. Hayward Union High School, Hayward. Calif. 1456 Glen Dr., San Leandro, Calif.

Rainich, G. Y. Prof., Univ. of Michigan, Ann Arbor, Mich. 602 Oswego St. 
Rainville, E. D. Asst. Prof., Univ. of Michigan, Ann Arbor, Mich. 1459 Rosewood, R.F.D. 4.

Rambo, Susan M. Prof., Smith Coll., Northampton, Mass. 12 Barrett P1.

Ramler, O. J. Prof., Catholic Univ. of America, Washington, D.C. 12 Girard St. N.E.

Ramsey, N. F. Asst. Prof., Physics Dept., Columbia Univ., New York, N.Y. Research Asso., Radiation Lab., Massachusetts Inst. of Tech., Cambridge, Mass. 4 Farrar St.

Randels, w. C. Asso. Prof., Univ. of Oklahoma, Norman, Okla. Faculty Exch.

Randolph, J. F. Asst. Prof., Cornell Univ., Ithaca, N.Y. White Hall.

Rankin, J. M. Prof., Coll. of Idaho, Caldwell, Idaho. 1810 Ash St.

Rao, K. N. Lib., Annamalai Univ., Annamalainagar, India.

Rasmusen, Dr. Ruth B. Instr., Wilson Junior Coll., Chicago, I11. 6105 Woodlawn Ave.

Rasor, S. E. Prof., Ohio State Univ., Columbus, Ohio. 1594 Neill Ave.

Rauch, L. L. Fellow, Princeton Univ., Princeton, N.J.

Rauch, Dr. L. M. Seton Hall Coll., South Orange, N.J.

Rauch, S. E. Santa Barbara State Coll., Santa Barbara, Calif.

Raudenbush, H. W. Asst. Prof., Queens Coll., Flushing, N.Y. 84-41 169th St., Jamaica, N.Y.

Rawhouser, Robert. Fort Hill High School, Cumberland, Md. 409 Louisiana Ave.

Rawlins, C. H. Prof., Postgraduate School, U.S. Naval Acad., Annapolis, Md. 13 Franklin St.

Raybould, Ethel H. Lecturer, Univ. of Queensland, Brisbane, Australia.

Rayl, Adrienne S. Asst. Prof., Birmingham Center, Univ. of Alabama, Birmingham, Ala. 1214 N. 32d St.

Raymond, J. H. Instr., St. Martin's Coll., Lacey, Wash.

Raynor, G. E. Asso. Prof., Lehigh Univ., Bethlehem, Pa. 530 10th Ave.

Reade, Dr. Maxwell. Instr., Purdue Univ., Lafayette, Ind.

Reagen, L. M. Asst. Prof., Polytech. Inst. of Brooklyn, Brooklyn, N.Y.

Reaves, S. W. Dean Emeritus and Prof., Univ. of Oklahoma, Norman, Okla.

ReBarker, Herbert. Prof., East Carolina Teachers Coll., Greenville, N.C.

Rechard, O. H. Prof., Univ. of Wyoming, Laramie, Wyo.

$\nmid$ Reddick, H. W. Prof., Cooper Union, New York, N.Y. Dir., Defense Training Inst., 375 Pearl St., Brooklyn, N.Y.

Reed, F. W. Prof., Ohio Univ., Athens, Ohio. 61 Columbia Ave.

Reed, L. J. Prof., School of Hygiene, Johns Hopkins Univ., Baltimore, Md. 615 N. Wolfe St.

Rees, C. J. Prof., Univ. of Delaware, Newark, Del.

Rees, Mina. Asso. Prof., Hunter Coll., Bedford Park Blvd. and Navy Ave., New York, N.Y. 140 E. 40th St.

Rees, P. K. Asst. Prof., Southern Methodist Univ., Dallas, Tex.

Regan, Francis. Asso. Prof., St. Louis Univ., St. Louis, Mo.

Rehberg, C. F. Instr. in Electrical Engineering, New York Univ., New York, N.Y. 25-28 84th St., Jackson Heights, N.Y.

Reichelderfer, Dr. P. V. Instr., Univ. of Chicago, Chicago, Ill.

Reid, W. T. Asso. Prof., Univ. of Chicago, Chicago, I11. Eckhart Hall.

Reingold, Dr. Haim. Instr., Our Lady of Cincinnati Coll., Cincinnati, Ohio. Illinois Inst. of Tech., Chicago, Ill. 146 N. Parkside Ave.

Reinsch, B. P. Prof., Florida Southern Coll., Lakeland, Fla. 191 Lake Morton Dr.

Reissner, Eric. Asst. Prof., Massachusetts Inst. of Tech., Cambridge, Mass.

Remick, B. L. Prof., Kansas State Coll. of Agric. and Applied Science, Manhattan, Kan. 613 Houston St.

Rempfer, Dr. R. W. Instr., Rensselaer Polytech. Inst., Troy, N.Y. Carnegie Hall. Reschovsky, Dr. Helene. Baldwin School, Bryn Mawr, Pa.

Reynolds, C. N. Prof., West Virginia Univ., Morgantown, W.Va. 217 McLane Ave.

Reynolds, Dr. F. G. Retired. 75 Central Park W., New York, N.Y.

Rhodes, E. E. 300 Broadway, Newark, N.J.

Riblet, H. J. Asst. Prof., Hofstra Coll., Hempstead. N.Y. Research Asso., Radiation Lab., Massachusetts Inst. of Tech., Cambridge, Mass. 26 Concord Ave.

Rice, Harris. Prof., Worcester Polytech. Inst., Worcester, Mass. 
Rice, J. N. Asso. Prof., Catholic Univ. of America, Washington, D.C. 3326 13th St. N.E.

Rich, G. R. Chief Design Engineer, Tennessee Valley Authority, 305 Union Bidg., Knoxville, Tenn.

Richardson, A. V. Prof., Bishop's Coll., Lennoxville, Quebec, Canada.

Richardson, D. P. Asso. Prof., Univ. of Arkansas, Fayetteville, Ark. Cleveland Ave. Capt., U.S.A.

Richardson, Dr. Moses. Instr., Brooklyn Coll., Brooklyn, N.Y.

$\nmid$ Richardson, R. G. D. Dean, Graduate School, Brown Univ., Providence, R.I. 44 University Ave.

Richert, D. H. Prof., Bethel Coll., North Newton, Kan.

Richmond, C. A. Retired. Tyngsboro, Mass. Instr., U.S.A., Signal Corps, Harvard Univ., Cambridge, Mass.

Richmond, D. E. Prof., Williams Coll., Williamstown, Mass. Mt. Pleasant.

$\nmid$ Richmond, Dr. H. W. Fellow, King's Coll., Univ. of Cambridge, Cambridge, England.

Rickart, Dr. C. E. Instr., Harvard Univ., Cambridge, Mass. 5 Crawford St.

Ricker, Dr. N. (H.) Senior Geophysicist, Geophysical Research Labs., Carter Oil Co., Tulsa, Okla. 1718 E. 30th St.

Rickey, F. A. Asso. Prof., Louisiana State Univ., University, La.

Rider, P. R. Prof., Washington Univ., St. Louis, Mo.

Riess, J. K. Asst., Physics Dept., Brown Univ., Providence, R.I.

Riesz, Frederick. Prof., Univ. of Szeged, Szeged, Hungary. 2 Baross-u.

Rietz, H. L. Retired. State Univ. of Iowa, Iowa City, Iowa. Physics Bldg.

Rigby, Dr. F. D. Instr., Texas Tech. Coll., Lubbock, Tex.

Rinehart, R. F. Asst. Prof., Case School of Applied Science, Cleveland, Ohio.

Rines, David. Patent Attorney, 739 First National Bank Bldg., 1 Federal St., Boston, Mass.

Riordan, John. Tech. Staff, Bell Telephone Labs., 463 West St., New York, N.Y.

Ripandelli, J. S. 35 Summer St., Forest Hills, N.Y. U.S.A., Camp Croft, S.C.

Ritt, J. F. Prof., Columbia Univ., New York, N.Y.

$\S$ Ritter, E. K. Asst. Instr., Univ. of Pennsylvania, Philadelphia, Pa. Lt. (j.g.), U.S.N.R., Postgraduate School, U.S. Naval Acad., Annapolis, Md.

Ritter, Dr. I. F. Instr., Coll. of Engineering, New York Üniv., University Heights, New York, N.Y.

Robbins, Dr. H. E. Instr., New York Univ., University Heights, New York, N.Y.

Robbins, Dr. R. B. Vice Pres. and Sec., Teachers Ins. and Annuity Asso., 522 5 th Ave., New York, N.Y.

Roberts, J. H. Asso. Prof., Duke Univ., Durham, N.C. Lt., U.S.N.R.

Roberts, W. C. Instr., Glendale Junior Coll., Glendale, Calif. 403 E. Fairview Ave.

Robertson, H. P. Prof., Princeton Univ., Princeton, N.J. 175 Prospect Ave.

Robertson, M. S. Asst. Prof., Rutgers Univ., New Brunswick, N.J.

Robertson, W. M. 41 Narbrook Park, Narberth, Pa.

Robinson, Dr. C. V. Instr., Univ. of Notre Dame, Notre Dame, Ind.

Robinson, G. de B. Asst. Prof., Univ. of Toronto, Toronto, Ont., Canada. Research Officer, National Research Council, Ottawa, Ont., Canada. 151 Metcalfe St.

Robinson H. A. Prof., Agnes Scott Coll., Decatur, Ga. Maj., U. S. Military Acad., West Point, N.Y.

§Robinson, Julia B. (Mrs. R. M.). 2633 Durant Ave., Berkeley, Calif.

$\S R o b i n s o n$, L. B. 131 E. North Ave., Baltimore, Md.

Robinson, L. V. Asst. Prof., Univ. of Mississippi, University, Miss.

Robinson, P. G. Asso. Prof., Iowa State Coll., Ames, Iowa.

Robinson, Robin. Prof., Dartmouth Coll., Hanover, N.H. 16 Allen St.

Robinson, R. M. Asst. Prof., Univ. of California, Berkeley, Calif.

Robinson, Dr. S. L. Instr., Coll. of the City of New York, 139th St. and Convent Ave., New York, N.Y. 4138 Westmoreland St., Little Neck, N.Y.

Robinson, Dr. V. N. Instr., Louisiana State Univ., University, La. 27 15th St. N.E., Atlanta, Ga. Lt. (j.g.), U.S.N.R., Amphibian Force, Norfolk, Va.

Robinson, W. J. Asst. Prof., Washington Coll., Chestertown, Md.

Rochford, Sister M. de Pazzi. Briar Cliff Coll., Sioux City, Iowa. 
Rock, Dr. D. H. Instr., Univ. of Nebraska, Lincoln, Neb. Fellow, School of Mech., Brown Univ., Providence, R.I.

Rodabaugh, L. D. Asst. Prof., Butler Univ., Indianapolis, Ind.

$\S R o d d$, T. E. Asst., Univ. of Wisconsin, Madison, Wis.

Rodgers, T. G. Dean Emeritus, New Mexico State Normal Univ., Las Vegas, N.Mex. 1018 4th St.

†Roe, Dr. Josephine R. (Mrs. E. D.). Retired. 123 W. Ostrander Ave., Syracuse, N.Y.

Roessler, E. B. Asst. Prof. and Asst. Statistician, Coll. of Agric., Univ. of California, Davis, Calif.

Roever, W. H. Prof., Washington Univ., St. Louis, Mo.

Rogers, F. W. Asst. Prof., Univ. of Cincinnati, Cincinnati, Ohio.

Roos, Dr. C. F. 500 5th Ave., New York, N.Y.

Roosevelt, G. E. Roosevelt and Son, 30 Pine St., New York, N.Y.

Root, R. E. Prof., U. S. Naval Acad., Annapolis, Md. 7 Franklin St.

Rorer, Dr. J. T. Lecturer, Temple Univ., Philadelphia, Pa. 333 N. 34th St.

Rosenbach, J. B. Prof., Carnegie Inst. of Tech., Pittsburgh, Pa. 2550 Beechwood Blvd.

Rosenbaum, Dr. Benjamin. Milford School, Milford, Conn. 1251 Boulevard, New Haven, Conn.

Rosenbaum, Ira. U.S.A., Branch Immaterial Replacement Training Center, Fort McClellan, Ala.

Rosenbaum, Dr. Louise Johnson (Mrs. R. A.). Instr., Reed Coll. Portland, Ore.

Rosenbaum, R. A. Instr., Reed Coll., Portland, Ore.

Rosenblatt, Alfred. Prof., Univ. of San Marcos, Lima, Peru. Calle Atahualpa 192, Miraflores, Peru.

Rosenbloom, J. H. Junior Physicist, Fire Control Lab., U. S. Gov't Service, Philadelphia, Pa. 2913 Tulip St.

§Rosenbloom, P. C. Asst., Stanford Univ., Stanford University, Calif.

Rosenthal, Arthur. Asst. Prof., Univ. of New Mexico, Albuquerque, N.Mex. 314 S. Maple St.

Rosenthal, Dr. Jenny E. Physicist, U.S.A. Signal Corps, Radar Lab., Fort Monmouth, N.J.

Ross, A. E. Asst. Prof., St. Louis Univ., St. Louis, Mo. 1342 McCutcheon, Richmond Heights, St. Louis, Mo.

Rosser, J. B. Asso. Prof., Cornell Univ., Ithaca, N.Y. White Hall.

Rosskopf, Dr. M. F. John Burroughs School, 755 S. Price Road, Clayton, Mo.

Roth, S. G. Instr., Washington Square Coll., New York Univ., New York, N.Y.

Roth, W. E. Asso. Prof., Univ. of Wisconsin at Milwaukee, Milwaukee, Wis.

Rothe, E. H. Prof., William Penn Coll., Oskaloosa, Iowa.

Rothrock, D. A. Prof. Emeritus, Indiana Univ., Bloomington, Ind. 1000 Atwater Ave.

Rowe, C. H. Prof., Univ. of Dublin, Dublin, Ireland. 38 Trinity Coll.

Rowland, S. A. Prof., Ohio Wesleyan Univ., Delaware, Ohio. 49 Oak Hill Ave. Lt. Col., U.S.A., New York Port of Embarkation, Brooklyn, N.Y.

Royall, N. N. Asso. Prof., Winthrop Coll., Rock Hill, S.C. Lt. (j.g.), U.S.N.R., Communications Office, Navy Yard, U.S. Navy Dept., Charleston, S.C.

Ruger, Dr. H. A. Retired. 526 N. Main St., Wellington, Ohio.

Ruley, B. T. Fellow, School of Mech. and Instr., Engineering Dept., Brown Univ., Providence, R. I.

Rulon, P. J. Asso. Prof., Education Dept., Harvard Univ., Cambridge, Mass. 13 Kirkland St.

Runge, Lulu L. Asst. Prof., Univ. of Nebraska, Lincoln, Neb.

Running, T. R. Prof. Emeritus, Univ. of Michigan, Ann Arbor, Mich. 1019 Michigan Ave.

Rupp, C. A. Prof., Pennsylvania State Coll., State College, Pa.

Rusk, W. J. Retired, 1415 Park St., Grinnell, Iowa.

Russell, Helen G. Asst. Prof., Wellesley Coll., Wellesley, Mass.

Rust, Dr. W. M. Head of Geophysics Research, Humble Oil and Refining Co., Houston, Tex.

Rutt, N. E. Prof., Louisiana State Univ., University, La.

$\S$ Ryan, R. A. Treas., Western and Southern Life Ins. Co., 4th and Broadway, Cincinnati, Ohio. Box 869. 
§Saastad, Arthur. Asst., Northwestern Univ., Evanston, I11.

Sabin, Mary S. 1333 E. 10th Ave., Denver, Colo.

Sadowsky, M. A. Asst. Prof., Illinois Inst. of Tech., Chicago, I11.

$\uparrow$ Safford, F. H. Prof. Emeritus, Univ. of Pennsylvania, Philadelphia, Pa. 4527 Osage Ave.

Saibel, E. A. Asso. Prof. of Mech., Carnegie Inst. of Tech., Pittsburgh, Pa. Engineer, Waco Aircraft Co., Troy, Ohio.

Salem, Dr. Raphael. Lecturer, Massachusetts Inst. of Tech., Cambridge, Mass.

§Samuelson, Dr. Hans. Univ. of Wyoming, Laramie, Wyo.

Samuelson, P. A. Asst. Prof., Economics Dept., Massachusetts Inst. of Tech., Cambridge, Mass.

Sánchez-Díaz, Rafael. Prof., Coll. of Agric. and Mech. Arts, Univ. of Puerto Rico, Mayaguez, Puerto Rico. Box 231.

Sanders, S. T. Retired. Box 1322, Baton Rouge, La.

Sanders, S. T., Jr. Prof., Southwestern Louisiana Inst., Lafayette, La. 310 General Mouton St.

Sandidge, w. L. Texas Military Coll., Terrell, Tex. U.S.A., Air Forces, S.A.T.S., Decatur, Ala.

Sandt, J. E. Asst. Prof., Marietta Coll., Marietta, Ohio. U.S.A.

Sanger, R. G. Asst. Prof., Univ. of Chicago, Chicago, Iil. Eckhart Hall.

Sard, Arthur. Asst. Prof., Queens Coll., Flushing, N.Y. 146-19 Beech Ave.

Sarratt, C. M. Prof., Vanderbilt Univ., Nashville, Tenn.

Sasuly, Max. U. S. Social Security Board, Washington, D.C. 3815 Upton St. N.W.

Satterthwaite, Dr. F. E. Group Statistical Div., Aetna Life Ins. Co., Hartford, Conn.

Saunders, T. J. Prof., Univ. of San Francisco, San Francisco, Calif.

Savage, Dr. L. J. Instr., Cornell Univ., Ithaca, N.Y.

Scammon, R. E. Prof., Univ. of Minnesota, Minneapolis, Minn. 172 Bedford St. S.E.

Scarborough, J. B. Prof., U. S. Naval Acad., Annapolis, Md. Ferry Farms.

\$Schaaf, S. A. Univ. of California, Berkeley, Calif. 24 Wheeler Hall.

Schaefer, M. B. Scientist, International Pacific Salmon Fisheries Com., Univ. of Washington, Seattle, Wash. Ensign, U.S.N.R., D.C.O., 12th Naval District, San Francisco, Calif.

Schaeffer, A. C. Asst. Prof., Stanford Univ., Stanford University, Calif.

§Schafer, Dr. R. D. Ensign, U.S.N.R., U.S.S. Essex. Care of Postmaster, New York, N.Y.

Schart, Dr. W. J. Asst. Physicist, Aircraft Radio Lab., Wright Field, Dayton, Ohio. 425 Grand Ave.

$\S$ Schatten, Robert. U.S.A., Station Hospital, Fort Benning, Ga.

Scheffé, Dr. Henry. Instr., Princeton Univ., Princeton, N.J. Fine Hall.

Scheier, Rev. M. A. Prof., St. Bonaventure Coll., St. Bonaventure, N.Y.

Schelkunoff, Dr. S. A. Consultant in Electromagnetic Theory, Bell Telephone Labs., 463 West St., New York, N.Y.

Scherberg, Dr. M. G. Aerodynamicist, Vought-Sikorsky Aircraft, Stratford, Conn. 87 Locust St., Bridgeport, Conn.

Scherk, Dr. Peter. Asst., Indiana Univ., Bloomington, Ind.

\$Schilansky, Dr. Ivor. Univ. of Michigan, Ann Arbor, Mich.

Schilling, Dr. O. F. G. Instr., Univ. of Chicago, Chicago, Ill. Eckhart Hall.

Schmidt, O. H. Instr., Brown Univ., Providence, R.I.

†Schmiedel, Oscar. San Blas Ave., R.F.D. 1, Atascadero, Calif.

Schneckenburger. Dr. Edith R. Instr., Michigan State Normal Coll., Ypsilanti, Mich. $210 \mathrm{~N}$. Washington St.

Schneider, Sister M. Christopher. 45 Candler Ave., Highland Park, Mich.

Schoenberg, I. J. Asst. Prof., Univ. of Pennsylvania, Philadelphia, Pa. 19 S. Wycombe Ave., Lansdowne, P.

Schraut, K. C. Asst. Prof., Univ. of Dayton, Dayton, Ohio.

$\S$ Schuettler, Alberta. Instr., Wellesley Coll., Wellesley, Mass.

Schwartz, Dr. Abraham. Instr., Pennsylvania State Coll., State College, Pa.

Schwartz, Dr. H. M. Instr., Univ. of Illinois, Urbana, I11. 367 Math. Bldg.

Schweigert, Dr. G. E. Instr., Univ. of Missouri, Columbia, Mo. 
Schweitzer, Dr. A. R. 452 W. Oakdale Ave., Chicago, I11.

Schweitzer, E. O. Electrical Engineer, 1241 Waukegan Road, Northbrook, Ill.

Scott, Dr. W. M. Federal Bureau of Investigation, U. S. Dept. of Justice, Washington, D.C.

Scott, Dr. W. T. Instr., Northwestern Univ., Evanston, I11. $2 d$ Lt., A.U.S., Ordnance Replacement Training Center, Aberdeen Proving Ground, Md.

Sealander, C. E. Asst. Prof., Univ. of South Dakota, Vermillion, S.Dak.

Sedgewick, C. H. W. Asso. Prof., Univ. of Connecticut, Storrs, Conn.

Sedgewick, Dr. Rose W. (Mrs. C. H. W.). Storrs, Conn.

Seebeck, Dr. C. L. Instr., Randolph Field, Tex. Box 303, New Braunfels, Tex.

Seekins, C. W. Ensign, U.S.N.R., U. S. Naval Acad., Annapolis, Md. 114 Severn Ave., West Annapolis, Md.

$\S$ Seely, Dr. Caroline E. Willseyville, Tioga Co., N.Y.

Segal, Dr. I. E. Research Asst., Princeton Univ., Princeton, N.J.

Seidel, Wladimir. Asso. Prof., Univ. of Rochester, Rochester, N.Y. River Campus.

§Seidenberg, Abraham. Johns Hopkins Univ., Baltimore, Md.

Seiler, Brother Louis de LaSalle. Dean of Studies and Prof., St. Mary's Coll., Winona, Minn.

Sellew, G. T. Prof. Emeritus, Knox Coll., Galesburg, I11. 140 W. 8th St., Claremont, Calif.

Semple, J. G. Prof., King's Coll., Univ. of London, London, W. C. 2, England. The University, Bristol, 8, England.

Senior, Dr. J. K. Research Asso., Chemistry Dept., Univ. of Chicago, Chicago, I11. 5612 Kenwood Ave.

Serbin, Dr. Hyman. Chief Aerodynamicist, Fairchild Aircraft Co., Hagerstown, Md.

Seth, B. R. Prof., Hindu Coll., Delhi, India.

Sewell, W. E. Asst. Prof., Georgia School of Tech., Atlanta, Ga. Care of T. W. Sewell, Newnan, Ga.

†Shah, S. M. Lecturer, Muslim Univ., Aligarh, India. Jail Road.

Shamos, M. H. 45 Christopher St., New York, N.Y.

§Shand, William. Research Asst., California Inst. of Tech., Pasadena, Calif. 551 S. Hill Ave.

§Shanks, E. B. Asst., Univ. of Illinois, Urbana, I11. 160 Math. Bldg.

Shanks, Dr. M. E. Instr., Univ. of Missouri, Columbia, Mo. 208 Engineering Bldg.

Shannon, Dr. C. E. Bell Telephone Labs., 463 West St., New York, N.Y.

$\S$ Shapley, Harlow. Prof., Harvard Univ., Cambridge, Mass. Harvard Observ.

§Sharpe, F. R. Prof. Emeritus, Cornell Univ., Ithaca, N.Y. 57 Central Ave., Ocean City, N.J.

Sharpe, Dr. J. A. Chief Physicist, Stanolind Oil and Gas Co., Tulsa, Okla. Box 591. Special Advisor, National Defense Research Com.

Shaub, H. C. Prof., Washington and Jefferson Coll., Washington, Pa.

Shaw, A. N. Prof., McGill Univ., Montreal, Quebec, Canada. Physics Bldg.

Shaw, W. F. 1733 Ohio Ave., Mercedes, Tex.

§Sheedy, J. w. Asst. Prof., Michigan State Coll., East Lansing, Mich.

Sheffer, H. M. Prof., Philosophy Dept., Harvard Univ., Cambridge, Mass. Emerson Hall.

Sheffer, I. M. Prof., Pennsylvania State Coll., State College, Pa. 212 Sparks B1dg.

Sheldon, E. W. Prof., Univ. of Alberta, Edmonton, Alta., Canada.

$\S S h e p a r d$, E. I. Asso. Prof., Williams Coll., Williamstown, Mass.

Shephard, Dr. R. W. Instr., Univ. of California, Berkeley, Calif.

Sheridan, L. W. Prof., Coll. of Mt. St. Vincent, New York, N.Y.

$\S$ Sherman, Bernard. Johns Hopkins Univ., Baltimore, Md.

Sherman, Dr. Jacob. Test Lab., Navy Yard, U. S. Navy Dept., Philadelphia, Pa. 5822 Pentridge St.

Sherman, Dr. Seymour. Research Lab., Curtiss-Wright Corp., Buffalo, N.Y.

Sherwood, G. E. F. Prof., Univ. of California at Los Angeles, Los Angeles, Calif. 
Shewhart, Dr. W. A. Research Statistician, Bell Telephone Labs., 463 West St., New York, N.Y. 158 Lake Dr., Mountain Lakes, N.J.

Shiffman, Dr. Max. Instr., Coll. of the City of New York, 139th St. and Convent Ave., New York, N.Y. 110 E. 177th St., Bronx.

Shirk, J. A. G. Prof., Kansas State Teachers Coll., Pittsburg, Kan.

Shohat, J. A. Prof., Univ. of Pennsylvania, Philadelphia, Pa.600 S. Eagle Road, Manoa, $\mathrm{Pa}$.

Shonka, E. A. Instr., St. Procopius Coll., Lisle, Ill.

Shook, C. A. Asso. Prof., Lehigh Univ., Bethlehem, Pa. 1122 W. Broad St.

Shook, Dr. R. C. International Statistical Bureau, Inc., 70 5th Ave., New York, N.Y.

Short, R. L. Beaumont High School, St. Louis, Mo. 1234 Broadway, Quincy, I11.

Shreve, Dr. D. R. Stress Analyst, McDonnell Aircraft Corp., St. Louis, Mo. 7108 Dale Ave., Richmond Heights, St. Louis, Mo.

Shugert, S. P. Prof., Univ. of Pennsylvania, Philadelphia, Pa. College Hall.

Shulits, Samuel. 1st Lt., U.S.A., Corps of Engineers, Engineer Office, Charleston, S.C. Box 516 .

Shumway, R. R. Asst. Dean and Prof., Univ. of Minnesota, Minneapolis, Minn.

Shuster, C. N. Prof., New Jersey State Teachers Coll., Trenton, N.J.

Siceloff, L. P. Prof., Columbia Univ., New York, N.Y.

Sigley, D. T. Asso. Prof., Kansas State Coll. of Agric. and Applied Science, Manhattan, Kan. 1710 Fairchild.

Siller, Harry. Asst. Statistician, National Resources Planning Board, Washington, D.C. 42 Independence Ave. S.W.

Silverman, L. L. Prof., Dartmouth Coll., Hanover, N.H.

Simmons, D. H. Fellow, Univ. of California at Los Angeles, Los Angeles, Calif. 260 Aster St., Laguna Beach, Calif. Instr., U.S.A., Air Corps, Santa Ana, Calif.

Simmons, H. A. Asso. Prof., Northwestern Univ., Evanston, I11.

Simon, L. G. Statistician, 225 W. 34th St., New York, N.Y.

Simon, W. G. Vice Pres., Dean and Prof., Western Reserve Univ., Cleveland, Ohio.

Simond, Ruth G. Asso. Prof., Hampton Inst., Hampton, Va. 123 Orchard St., Franklin, N.H. U. S. Navy Dept.

Simons, Dr. Lao G. Retired. 875 West End Ave., New York, N.Y.

§Simons, W. H. Asst., Univ. of California, Berkeley, Calif.

Simpson, Harold. Prof., Bedford Coll., Univ. of London, London, N.W. 1, England.

§Simpson, R. C. Asst., Univ. of Wisconsin, Madison, Wis. North Hall.

Simpson, T. M. Prof., Univ. of Florida, Gainesville, Fla.

Sinclair, Mary E. Prof., Oberlin Coll., Oberlin, Ohio. 260 Oak St.

Singer, Dr. James. Instr., Brooklyn Coll., Brooklyn, N.Y. 3054 Bedford Ave.

Singer, Dr. P. M. Instr., Extension Div., Univ. of California, Berkeley, Calif. 1288 Campus Dr.

Singleton, H. E. 141 Anacostia Road S.E., Washington, D.C.

Singleton, R. R. Research Asso., Princeton Univ., Princeton, N.J. 20 Nassau St.

Sinkov, Dr. Abraham. Cryptanalyst, Signal Corps, Signal Office, Munitions Bldg., Washington, D.C. Maj., U.S.A.

†Sisam, C. H. Prof., Colorado Coll., Colorado Springs, Colo. 816 N. Weber St.

Slepian, Dr. Joseph. Asso. Dir., Research Labs., Westinghouse Electric and Mfg. Co., East Pittsburgh, Pa.

†Slichter, C. S. Dean Emeritus and Prof. Emeritus, Univ. of Wisconsin, Madison, Wis. $636 \mathrm{~N}$. Frances St.

Slobin, H. L. Dean, Univ. of New Hampshire, Durham, N.H.

Slotnick, Dr. M. M. Humble Oil and Refining Co., Houston, Tex. Supervisor, Ordnance Lab., Navy Yard, U. S. Navy Dept., Washington, D.C. 12 Ridge Road S.E.

Smail, L. L. Prof., Lehigh Univ., Bethlehem, Pa.

Smiley, C. H. Asso. Prof. of Astronomy, Brown Univ., Providence, R.I.

Smiley, Dr. Dorothy M. (Mrs. M. F.). 204 E. Market St., Bethlehem, Pa.

Smiley, M. F. Asst. Prof., Lehigh Univ., Bethlehem, Pa. 204 E. Market St. 
Smith, A. H. Asst. Prof., Purdue Univ., Lafayette, Ind.

Smith, A. J. Prof., Susquehanna Coll., Selinsgrove, $\mathrm{Pa}$.

Smith, Dr. Burke. Transmission Engineer, Illinois Bell Telephone Co., 212 W. Washington St., Chicago, I11. 5729 Kimbark Ave.

Smith, Dr. C. B. Instr., Montana State Coll., Bozeman, Mont.

†Smith, Clara E. Prof. Emeritus, Wellesley Coll., Wellesley, Mass. 14 Waban St.

Smith, C. W. Acting Pres., State Teachers Coll., Superior, Wis.

†Smith, D. E. Prof. Emeritus, Teachers Coll., Columbia Univ., New York, N.Y. 501 W. 120th St.

Smith, D. M. Prof., Georgia School of Tech., Atlanta, Ga.

Smith, E. R. Prof., Iowa State Coll., Ames, Iowa.

Smith, E. S. Prof., Univ. of Cincinnati, Cincinnati, Ohio. Maj., U.S.A., Engineer Depot Columbus, Ohio.

Smith, F. C. Prof., Coll. of St. Francis, Joliet, Ill.

Smith, Gertrude. Asso. Prof., Vassar Coll., Poughkeepsie, N.Y.

Smith, G. W. Prof., Univ. of Kansas, Lawrence, Kan. 1730 Illinois St.

Smith, Helen F. Instr., Iowa State Coll., Ames, Iowa. 113 Welch Ave.

Smith, H. L. Prof., Louisiana State Univ., University, La. Nicholson Hall.

Smith, H. W. Asso. Prof., Oklahoma Agric. and Mech. Coll., Stillwater, Okla.

Smith, I. W. Prof. Emeritus, North Dakota State Coll., Fargo, N.Dak. Actuary, Ancient Order of United Workmen of North Dakota, 203 10th St. N., Fargo, N.Dak.

Smith, J. C. Instr., Cornell Univ., Ithaca, N.Y.

Smith, Rev. J. P. Church of Gesu, 18th and Thompson Sts., Philadelphia, Pa.

Smith, P. A. Asso. Prof., Columbia Univ., New York, N.Y.

Smith, R. E. Prof., Atlantic Christian Coll., Wilson, N.C. 110 N. Kincaid Ave.

Smith, R. G. Prof., Kansas State Teachers Coll., Pittsburg, Kan.

Smith, S. S. Asst. Prof., Univ. of Utah, Salt Lake City, Utah.

Smith, T. L. Asst. Prof., Carnegie Inst. of Tech., Pittsburgh, Pa.

Smith, W. F. Prof., West Virginia Inst. of Tech., Montgomery, W.Va. Box 788.

Smith, W. H. 320 T St. N.W., Washington, D.C.

Smith, W. M. Prof., Lafayette Coll., Easton, Pa.

Smith, W. N. Asst., Univ. of Wisconsin, Madison, Wis.

Smithies, Dr. Frank. Fellow, St. John's Coll., Univ. of Cambridge, Cambridge, England. Experimental Officer, Ministry of Supply, London, England.

Snapper, Dr. Ernst. Instr., Princeton Univ., Princeton, N.J. 82 Linden Lane.

Snook, T. E. Architect, 299 Broadway, New York, N.Y.

Snow, Dr. Chester. Senior Physicist, National Bureau of Standards, U.S. Dept. of Commerce, Washington, D.C. 110 Radio Bldg.

§Snowdon, S. C. Fellow, Physics Dept., California Inst. of Tech., Pasadena, Calif.

Snyder, A. D. Asso. Prof., Union Coll., Schenectady, N.Y. 1592 Union St.

§Snyder, Virgil. Prof. Emeritus, Cornell Univ., Ithaca, N.Y. 214 University Ave. Visiting Prof., Brown Univ., Providence, R.I.

Snyder, Dr. W. S. Instr., Illinois Inst. of Tech., Chicago, I11. 5649 S. Drexel Ave.

Sobczyk, Dr. Andrew. Instr., Oregon State Coll., Corvallis, Ore. Radiation Lab., Massachusetts, Inst. of Tech., Cambridge, Mass.

§Sohl, H. K. Fellow, School of Mech., Brown Univ., Providence, R.I.

Sohn, Harry. No address.

Sohon, Rev. F. W. Dir., Seismological Observ., and Prof., Georgetown Univ., Washington, D.C.

Sokolnikoff, Dr. Elizabeth S. (Mrs. I. S.). Lecturer, Univ. of Wisconsin, Madison, Wis. 1111 Oak Way, Shorewood.

Sokolnikoff, I. S. Prof., Univ. of Wisconsin, Madison, Wis. North Hall.

Solomon, Herbert. Statistician, Quartermaster Depot, Jersey City, N.J. 801 E. Tremont Ave., Bronx, New York, N.Y.

Solt, M. R. Asst. Prof., Univ. of New Hampshire, Durham, N.H. Box 283.

Sorgenfrey, Dr. R. H. Instr., Case School of Applied Science, Cleveland, Ohio.

Southard, Dr. T. H. Instr., Wayne Univ., Detroit, Mich. Statistician, War Production Board, Washington, D.C. 1504 Seminary Road, Silver Spring, Md. 
Spear, Joseph. Prof., Northeastern Univ., Boston, Mass. 360 Huntington Ave.

Specht, Dr. R. D. Instr., Univ. of Florida, Gainesville, Fla.

Speeker, G. G. Asso. Prof., Michigan State Coll., East Lansing, Mich. Box 861.

Speer, Dr. Mary T. (Mrs.). Instr., Univ. of Pittsburgh, Pittsburgh, Pa.

Spencer, D. C. Asso. Prof., Stanford Univ., Stanford University, Calif.

Spencer, Domina E. Asst. Prof., American Univ., Washington, D.C. 106 Hurst Hall.

Spencer, Dr. Vivian E. Senior Mineral Economist, U.S. Dept. of Commerce, Washington, D.C. Room 5122, Commerce Bldg.

Sperry, Pauline. Asso. Prof., Univ. of California, Berkeley, Calif. 28 Wheeler Hall.

Spicer, C. A. Prof., Western Maryland Coll., Westminster, Md. 17 Ridge Road.

Spinks, M. J. 425 W. Main St., Wilmington, Ohio.

Spitzbart, Dr. Abraham. Instr., Univ. of Minnesota, Minneapolis, Minn. 126 Folwell Hall.

Spooner, C. C. Prof., Northern Michigan Coll. of Education, Marquette, Mich. 117 E. Ridge St.

Sprague, A. H. Prof., Amherst Coll., Amherst, Mass. 227 S. Pleasant St.

Springer, C. E. Prof., Univ. of Oklahoma, Norman, Okla.

$\S$ Springer, M. D. Asst., Univ. of Illinois, Urbana, I11. 160 Math. Bldg.

Stabler, E. R. Asst. Prof., Hofstra Coll., Hempstead, N.Y.

§Staderman, O. P. Asst., Univ. of Illinois, Urbana, I1l. 160 Math. Bldg.

Stafford, Anna A. Asst. Prof., Univ. of Utah, Salt Lake City, Utah.

Stanwick, C. A. Electrical Engineer. 131 Rynda Road, South Orange, N.J.

Starcher, G. W. Prof., Ohio Univ., Athens, Ohio. R.F.D. 3.

Stark, Marion E. Asso. Prof., Wellesley Coll., Wellesley, Mass. 6 Waban St.

Stark, R. H. Instr., Northwestern Univ., Evanston, Ill.

Starke, E. P. Asso. Prof., Rutgers Univ., New Brunswick, N.J.

Starr, Dr. D. W. Instr., Southern Methodist Univ., Dallas, Tex.

Stauffer, J. R. K. Kingston, R.I.

§Stechschulte, V. C. Prof., Xavier Univ., Cincinnati, Ohio.

Stecker, Ormelle (Mrs.). Asst. Prof., Pennsylvania State Coll., State College, Pa. 306 Miles St.

Steed, D. V. Asso. Prof., Univ. of Southern California, Los Angeles, Calif.

Steen, F. H. Asst. Prof., Georgia School of Tech., Atlanta, Ga. 532 Peeples St. S.W.

Steenrod, N. E. Asst. Prof., Univ. of Chicago, Chicago, I11.

Steinhaus, Dr. H. W. Chief, Research Div., Group Dept., Equitable Life Assurance Society of the U.S., 393 7th Ave., New York, N.Y. Box 386, Scarsdale, N.Y.

Stelson, H. E. Prof., Kent State Univ., Kent, Ohio.

Stephan, F. F. Prof. of Sociology and Statistics, Cornell Univ., Ithaca, N.Y. Chief, Statistical Service, War Manpower Com., Washington, D.C. 4924 Little Falls Road, Arlington, Va.

Stephany, E. O. 92 Tyler St., Rochester, N.Y.

Stephens, R. C. Prof., Knox Coll., Galesburg, I11.

Stephens, R. P. Dean, Graduate School, Univ. of Georgia, Athens, Ga.

\$Sternberg, Dr. Wolfgang. 17 Francis Ave., Cambridge, Mass.

†Stetson, J. M. Prof., Coll. of William and Mary, Williamsburg, Va.

Stevenson, Guy. Prof., Univ. of Louisville, Louisville, Ky.

Stewart, Dr. B. M. Instr., Michigan State Coll., East Lansing, Mich. 705 Grove St.

§Stewart, F. M. Fellow, Harvard Univ., Cambridge, Mass. 31 Conant Hall.

§Stewart, J. C. Asst., Univ. of Illinois, Urbana, I11.

Stewart, S. W. Junior Radio Engineer, U.S.A., Signal Corps, Radar Lab., Fort Monmouth, N.J. 503 W. Oliver St., Owosso, Mich.

Stibitz, Dr. G. R. Mathematician, Bell Telephone Labs., 463 West St., New York, N.Y. 748 Green St., Boonton, N.J. Prin. Tech. Aide, National Defense Research Com.

Stoker, J. J. Asso. Prof., New York Univ., New York, N.Y. 66 Wayne Ave., White Plains, N.Y. 
Stokes, C. N. Prof., Temple Univ., Philadelphia, Pa. 6416 N. Camac St.

Stokes, Dr. Ellen C. Instr., New York State Coll. for Teachers, Albany, N.Y.

Stokes, Ruth W. Prof., Winthrop Coll., Rock Hill, S.C. Box 113.

Stoll, R. R. Instr., Williams Coll., Williamstown, Mass.

§Stone, Dr. A. H. Inst. for Advanced Study, Princeton, N.J. Fuld Hall.

Stone, M. H. Prof., Harvard Univ., Cambridge, Mass. 30 Hillside Ave.

Stone, R. B. Asso. Prof. and Registrar, Purdue Univ., Lafayette, Ind. 615 Russell St., West Lafayette, Ind.

Stopher, Dr. E. C. Brockport State Normal School, Brockport, N.Y.

Story, Helen F. Instr., St. Petersburg Junior Coll., St. Petersburg, Fla.

Stouffer, E. B. Dean, Univ. of Kansas, Lawrence, Kan. 1019 Maine St.

§Strange, W. J. Asst., Yale Univ., New Haven, Conn. 323 Harkness.

Stratton, W. T. Prof., Kansas State Coll. of Agric. and Applied Science, Manhattan, Kan.

§Straus, E. G. Fellow, Columbia Univ., New York, N.Y. 98 Thayer St.

Strayhorn, Elizabeth C. Asst. Prof., Western Kentucky State Teachers Coll., Bowling Green, Ky. W.A.A.C., Fort Des Moines, Iowa.

Street, Dr. R. E. Asst. Physicist, National Advisory Com. for Aeronautics, Langley Field, Hampton, Va. 146 Hanover Ave.

Strobel, Dr. C. F. Instr., North Carolina State Coll., Raleigh, N.C.

Strodt, Ruth C. (Mrs. W. C.). 45 Tiemann P1., New York, N.Y.

Strodt, Dr. W. C. Instr., Columbia Univ., New York, N.Y. 45 Tiemann P1.

Struik, D. J. Prof., Massachusetts Inst. of Tech., Cambridge, Mass.

Struik, Dr. Saly R. R. (Mrs. D. J.). Fellow, Radcliffe Coll., Cambridge, Mass. 179 Oakley Road, Belmont, Mass.

§Stump, Margaret E. Asst., Indiana Univ., Bloomington, Ind. 127 Blue Ridge Road, Indianapolis, Ind.

Sturley, E. A. Berkshire School, Sheffield, Mass.

Sturm, Dr. R. G. Research Engineer Physicist, Aluminum Co. of America, New Kensington, Pa. Box 772.

Suffa, Mary C. Prof., Elmira Coll., Elmira, N.Y.

Sugar, Dr. Alvin. 2450 Blaine, Detroit, Mich. $2 \dot{d}$ Lt., U.S.A., Air Corps.

Sullivan, C. T. Prof., McGill Univ., Montreal, Quebec, Canada. Engineering Bldg.

Sullivan, Sister Helen. Prof., Mt. St. Scholastica Coll., Atchison, Kan.

Sullivan, Dr. Mildred M. Instr., Queens Coll., Flushing, N.Y.

Sunde, E. D. Engineer, Bell Telephone Labs., 463 West St., New York, N.Y.

Supnick, Fred. 4037 New Hampshire Ave. N.W., Washington, D.C.

Sutherland, Ethel. Asso., Teachers Coll., Columbia Univ., New York, N.Y.

Sutton, C. S. Asst. Prof., The Citadel, Charleston, S.C.

Sutton, Dr. Flora D. 308 Southway St., Baltimore, Md

Sutton, F. X. Instr., Suffolk Univ., Boston, Mass.

Swain, Dr. R. L. Instr., Univ. of Wisconsin, Madison, Wis.

Swann, Dr. W. F. G. Dir., Bartol Research Foundation, Franklin Inst., Swarthmore, $\mathrm{Pa}$.

Swanson, L. W. Asst. Prof., Coe Coll., Cedar Rapids, Iowa.

†Swift, Elijah. Dean and Prof., Univ. of Vermont and State Agric. Coll., Burlington, Vt. $415 \mathrm{~S}$. Willard St.

Swift, J. D. 1st Lt., U.S.A., Camp Bowie, Tex.

Swinford, Dr. L. H. Instr., Univ. of California, Berkeley, Calif. 1619 Oxford St.

Swingle, P. M. Asso. Prof., New Mexico Coll. of Agric. and Mech. Arts, State College, N.Mex. Box 277.

Swope, W. G. Instr., Yale Univ., New Haven, Conn. 32 N. Sheffield Hall.

Synge, J. L. Prof., Univ. of Toronto, Toronto, Ont., Canada.

Szász, Dr. Otto. Research Lecturer, Univ. of Cincinnati, Cincinnati, Ohio.

§Szatrowski, Dr. Zenon. Instr., Economics Dept., Northwestern Univ., Evanston, I11. $1762 \mathrm{r} / 2$ Hinman Ave.

Szegö, Gabor. Prof., Stanford Univ., Stanford University, Calif.

Takasu, Tsurusaburo. Prof., Tóhoku Imperial Univ., Sendai, Japan. Math. Inst., Coll. of Science. 
Tamarkin, J. D. Prof., Brown Univ., Providence, R.I.

Tanzola, J. J. Chief, Pension Div., Dept. of Finance, New York, N.Y. 2041 Watson Ave., Bronx.

Tappan, A. Helen. Dean and Prof., Western Coll., Oxford, Ohio.

Tarski, Dr. Alfred. Lecturer, Univ. of California, Berkeley, Calif.

Taub, A. H. Asst. Prof., Univ. of Washington, Seattle, Wash. Palmer Physical Lab., Princeton Univ., Princeton, N.J.

Taussky, Dr. Olga (Mrs. John Todd). Lecturer, Westfield Coll., Univ. of London, Oxford, England. St. Peter's Hall.

Taylor, A. E. Asst. Prof., Univ. of California at Los Angeles, Los Angeles, Calif.

Taylor, B. P. Instr., Extension Div., Univ. of California at Los Angeles, Los Angeles, Calif. 11326 Gladwin St., West Los Angeles, Calif. Senior Mathematician, Douglas Aircraft Co., El Segundo, Calif.

Taylor, Eugene. Prof., Univ. of Idaho, Moscow, Idaho.

Taylor, J. H. Prof., George Washington Univ., Washington, D.C.

Taylor, J. S. Prof., Univ. of Pittsburgh, Pittsburgh, Pa.

Taylor, Mildred E. Prof., Mary Baldwin Coll., Staunton, Va.

Taylor, Dr. W. C. Instr., Univ. of Cincinnati, Cincinnati, Ohio.

Tepping, Dr. B. J. Asso. Statistician, Bureau of the Census, U. S. Dept. of Commerce, Washington, D.C.

Terracini, Alejandro. Prof., Univ. of Tucumán, Tucumán, Argentina. Calle Salta 417.

Terry, M. E. 14 Elm St., Springfield, Vt. R.C.A.

Thaxton, H. M. Prof., Agric. and Tech. Coll. of North Carolina, Greensboro, N.C. Box 1074 .

Theobald, John. Prof., Loras Coll. of Dubuque, Dubuque, Iowa.

Thielman, H. P. Prof., Coll. of St. Thomas, St. Paul, Minn.

Thomas, C. F. Prof., Case School of Applied Science, Cleveland, Ohio.

Thomas, Dr. G. B. Instr., Massachusetts Inst. of Tech., Cambridge, Mass.

Thomas, J. M. Prof., Duke Univ., Durham, N.C. 4785 Duke Station.

Thomas, Sister M. Placide. Pres., Mt. St. Agnes Junior Coll., Mt. Washington, Baltimore, Md.

Thomas, T. Y. Prof., Univ. of California at Los Angeles, Los Angeles, Calif.

Thompson, E. L. Prof., Texas Tech. Coll., Lubbock, Tex.

Thompson, J. E. Prof., Pratt Inst., Brooklyn, N.Y. 183 Steuben St.

Thompson, Dr. J. M. Western Div., Agric. Adjustment Admin., U.S. Dept. of Agric., Washington, D.C.

Thompson, J. S. Vice Pres., Mutual Benefit Life Ins. Co., 300 Broadway, Newark, N.J.'

Thompson, Dr. W. R. 883 Warren St., Albany, N.Y.

Thomson, J. F. Asst. Prof., Tulane Univ., New Orleans, La.

Thorne, Dr. C. J. Instr., Univ. of Michigan, Ann Arbor, Mich.

Thorne, H. H. Lecturer, Univ. of Sydney, Sydney, Australia.

Thornton, Dr. Marian W. (Mrs.). Statistician, Minnesota Dept. of Health, Minneapolis, Minn. 852 Warren St., Albany, N.Y.

Thrall, R. M. Asst. Prof., Univ. of Michigan, Ann Arbor, Mich. 319 W. Engineering Bldg.

Thron, W. J. Fellow, Rice Inst., Houston, Tex.

Thuener, Sister M. Domitilla. Prof., Villa Madonna Coll., Covington, Ky. Villa Madonna Convent, Box 33, R.F.D. 2.

Thullen, Dr. Peter. Asst. Dir., Actuarial Dept., Inst. Nacional de Previsión, Quito, Ecuador. Apartado 636.

Thurman, Dr. G. R. Instr., Univ. of Missouri, Columbia, Mo. 208 Engineering Bldg.

Thurston, H. S. Asso. Prof., Univ. of Alabama, University, Ala.

Tilley, Arthur. Asso. Prof., Washington Square Coll., New York Univ., New York, N.Y.

Timoshenko, S. P. Prof., Stanford Univ., Stanford University, Calif. 536 W. Crescent Dr., Palo Alto, Calif.

Tintner, Gerhard. Asso. Prof., Economics Dept., Iowa State Coll., Ames, Iowa. 
Titt, E. W. Asso. Prof., Univ. of Texas, Austin, Tex. 2206 Newfield Lane.

Tolar, Blanche H. (Mrs. M. B.). Asst. Prof., Fenn Coll., Cleveland, Ohio. 3124 E. Overlook Road, Cleveland Heights, Ohio.

Tolle, L. F. Prof., Teachers Coll., St. John's Univ., Brooklyn, N.Y. 240-23 141st Ave., Rosedale, N.Y.

Tolman, R. C. Dean, Graduate School, California Inst. of Tech., Pasadena, Calif.

Tolonen, E. O. Cost Auditor, U.S.A., Air Forces, Detroit, Mich. 15764 Kentucky Ave.

Tompkins, Dr. C. B. Instr., Princeton Univ., Princeton, N.J. Care of R. D. Tompkins, Mountain Home, Tenn. Lt. (j.g.), U.S.N.R.

Tonelli, Leonida. Prof., Univ. of Pisa, Pisa, Italy.

Toops, H. A. Prof., Ohio State Univ., Columbus, Ohio. 1430 Cambridge Blvd.

Topp, C. W. Instr., Fenn Coll., Cleveland, Ohio. 1946 Woodward Ave., Cleveland Heights, Ohio.

Tordella, L. W. Asst. Prof., Loyola Univ., Chicago, I11. 7431 Kenwood Ave. Lt. (j.g.), U.S.N.R., U.S. Navy Dept., Washington, D.C.

§Tornheim, Dr. Leonard. 4246 West End Äve., Chicago, Ill. U.S.A., Fort Sill, Okla.

Torrance, C. C. Asst. Prof., Case School of Applied Science, Cleveland, Ohio.

Torrance, Dr. Esther M. (Mrs. C. C.). 3607 Randolph Road, Cleveland Heights, Ohio.

Torrey, Marian M. Prof., Goucher Coll., Baltimore, Md.

Townsend, E. J. Prof. Emeritus, Univ. of Illinois, Urbana, I11. 510 E. John St., Champaign, Ill.

Tracey, J. I. Asso. Prof., Yale Univ., New Haven, Conn. 84 McKinley Ave.

Trachtenberg, Sidney. 2d Lt., U.S.A., Air Forces, Kelly Field, Tex.

Transue, Dr. W. R. Asst., Inst. for Advanced Study, Princeton, N.J. 9 Patton Ave.

Trapani, B. J. 1406 Farr St., Scranton, Pa. Capt., U.S.A., Camp Livingston, La.

Tremblay, Althéod. Prof., Laval Univ., Quebec, Quebec, Canada. 367 St. Cyrille St.

Tricomi, Francesco. Prof., Univ. of Turin, Turin, Italy. Via Po 15.

Trimble, Dr. H. C. Instr., Iowa State Teachers Coll., Cedar Falls, Iowa.

Tripp, M. O. Prof. Emeritus, Wittenberg Coll., Springfield, Ohio. 218 W. Cecil St.

§Tripp, Dr. R. H. Grumman Aircraft Engineering Corp., Bethpage, N.Y.

Trjitzinsky, W. J. Prof., Univ. of Illinois, Urbana, Ill.

Trott, G. R. Prof., Blue Mountain Coll., Blue Mountain, Miss.

§Truesdell, C. A. Instr., Princeton Univ., Princeton, N.J.

Trump, P. L. Asst. Prof., University High School, Univ. of Wisconsin, Madison, Wis.

Tryon, G. M. V. 105 W. Shiawassee Ave., Fenton, Mich.

Tuan, H. F. Princeton Univ., Princeton, N.J.

Tucker, A. W. Asso. Prof., Princeton Univ., Princeton, N.J. Fine Hall.

Tucker, C. B. Asst. Prof., Kansas State Teachers Coll., Emporia, Kan.

Tuckerman, Bryant. Cosmos Club, Washington, D.C.

Tukan, K. H. Prof. and Vice Prin., Najah National Coll., Nablus, Palestine. Box 31.

Tukey, J. W. Asst. Prof., Princeton Univ., Princeton, N.J. Fine Hall.

Tuller, Dr. Annita. Instr., Hunter Coll., Bedford Park Blvd. and Navy Ave., New York, N.Y. 139-62 Pershing Crescent, Jamaica, N.Y.

Tulloch, L. H. Instr., San Antonio Junior Coll., San Antonio, Tex.

§Turner, Dr. Alice E. Instr., Connecticut Coll., New London, Conn.

Turner, Bird M. Prof., West Virginia Univ., Morgantown, W.Va. 354 Spruce St.

Turner, Dr. Mary H. (Mrs. M. J.). 150 Washington Ter., Bridgeport, Conn.

Turquette, A. R. Instr., Cornell Univ., Ithaca, N.Y. 1 Sage Ave.

Turrittin, H. L. Asst. Prof., Univ. of Minnesota, Minneapolis, Minn. 4046 Beard Ave. S.

Uhler, H. S. Prof. Emeritus and Lecturer, Yale Univ., New Haven, Conn. 217 Prospect St.

Ulam, S. M. Asst. Prof., Univ. of Wisconsin, Madison, Wis. 
Ulrich, Dr. F. E. Instr., Rice Inst., Houston, Tex.

Umberger, E. H. Instr., Pennsylvania State Coll., State College, Pa. 511 E. Foster Ave.

Underhill, A. L. Asso. Prof., Univ. of Minnesota, Minneapolis, Minn. 126 Folwell Hall.

Upton, C. B. Prof. Emeritus, Teachers Coll., Columbia Univ., New York, N.Y.

Urban, F. W. Prof., Central Missouri State Teachers Coll., Warrensburg, Mo. 418 N. Maguire St.

Urner, Dr. S. E. Instr., Los Angeles City Coll., Los Angeles, Calif. 821 N. Mariposa Ave.

Uspensky, J. V. Prof., Stanford Univ., Stanford University, Calif. 511 Hanover St., Palo Alto, Calif.

Vaage, E. F. Telephone Engineer, Bell Telephone Labs., 463 West St., New York, N.Y.

Valentine, F. A. Asst. Prof., Univ. of California at Los Angeles, Los Angeles, Calif.

Vallarta, M. S. Prof., Physics Dept., Massachusetts Inst. of Tech., Cambridge, Mass.

Van Buskirk, H. C. Prof., California Inst. of Tech., Pasadena, Calif. 390 S. Ho1liston Ave.

Vance, E. P. Asst. Prof., Univ. of Nevada, Reno, Nev.

Vanderslice, J. L. Asst. Prof., Univ. of Maryland, College Park, Md.

Vandiver, H. S. Prof., Univ. of Texas, Austin, Tex.

Van Engen, Henry. Asst. Prof., Iowa State Teachers Coll., Cedar Falls, Iowa.

†Van Orstrand, Dr. C. E. Retired. 3906 Morrison St., Chevy Chase Branch, Washington, D.C.

Van Schaack, G. B. Asst. Prof., Michigan State Coll., East Lansing, Mich.

Van Vleck, E. B. Prof. Emeritus, Univ. of Wisconsin, Madison, Wis. 519 N. Pinckney St.

Van Vleck, J. H. Prof., Harvard Univ., Cambridge, Mass. Research Physics Lab.

Varineau, Dr. V. J. Ground School Instr., U.S. Naval Air Station, Pensacola, Fln. 700 E. Mallory St.

Vass, Dr. J. I. Instr., Univ. of Wisconsin at Milwaukee, Milwaukee, Wis.

Vassallo, Brother Godfrey. Prof., Univ. of Portland, Portland, Ore.

Vaudreuil, Sister M. Felice. Prof., Mt. Mary Coll., Milwaukee, Wis.

Vaughan, Dr. H. E. Asso., Univ. of I1linois, Urbana, I11. 907 S. Vine St.

† Veblen, Oswald. Prof., Inst. for Advanced Study, Princeton, N.J. 58 Battle Road.

Vest, M. L. Instr., West Virginia Univ., Morgantown, W.Va.

Vezeau, W. A. Instr., Univ. of Detroit, Detroit, Mich.

Vickery, Dr. C. W. Asso. Statistician, Public Roads Admin., Washington, D.C.

† Vijayaraghavan, Dr. Thirukkannapuram. Reader, Univ. of Dacca, Dacca, India.

$\S$ Vinograde, Dr. Bernard. Instr., Univ. of Wisconsin, Madison, Wis.

Virata, E. T. Prof., Univ. of the Philippines, Manila, Philippine Islands.

Vitale, R. L. Junior Electrical Engineer, Board of Transportation of the City of New York, New York, N.Y. $2 d$ Lt., A.U.S., Signal Corps, Radar School, Camp Murphy, Fla.

Votaw, D. F. Princeton Univ., Princeton, N.J. 73 Graduate Coll.

Waddell, Mary E. G. Univ. of Toronto, Toronto, Ont., Canada. 72 Madison Ave.

Wade, Dr. L. I. National Research Fellow. Inst. for Advanced Study, Princeton, N.J.

Wade, T. L. Asst. Prof., Univ. of Alabama, University, Ala. 2400 Glendale Gardens, Tuscaloosa, Ala.

Wagner, C. C. Prof., Pennsylvania State Coll., State College, Pa. 122 S. Atherton St.

Wagner, Dr. R. W. Lt. (j.g.), U.S.N.R., U. S. Naval Acad., Annapolis, Md.

Wagner, T. C. G. Instr., Univ. of Maryland, College Park, Md. 3728 Northampton St. N.W., Washington, D.C.

Wahlin, G. E. Prof., Univ. of Missouri, Columbia, Mo. 1401 Anthony St.

Wakerling, R. K. Asst. Prof., Fresno State Coll., Fresno, Calif. 
Wald, Abraham. Asst. Prof., Columbia Univ., New York, N.Y.

Walder, O. E. Asso. Prof., South Dakota State Coll., Brookings, S.Dak. State College Station.

Walker, B. M. Prof. Emeritus, Mississippi State Coll., State College, Miss.; Pres., West Point Oil Mill, Starkville, Miss.

$\S$ Walker, Dr. G. L. Instr., Univ. of Delaware, Newark, Del.

Walker, Rev. G. W. 47 Grove St., Perry, N.Y.

Walker, R. J. Asst. Prof., Cornell Univ., Ithaca, N.Y. White Hall.

Wall, H. S. Asso. Prof., Northwestern Univ., Evanston, I11.

Wallace, Dr. A. D. Instr., Univ. of Pennsylvania, Philadelphia, $\mathrm{Pa}$.

Wallace, Dr. P. R. Instr., Massachusetts Inst. of Tech. Cambridge, Mass. 17A Forest St.

Wallman, Dr. Henry. Radiation Lab., Massachusetts Inst. of Tech., Cambridge, Mass. 5 Crawford St.

Walmsley, Charles. Asso. Prof. Dalhousie Univ., Halifax, N.S., Canada. 6 Studley Ave.

Walsh, J. L. Prof., Harvard Univ., Cambridge, Mass. 474 Widener Lib. Lt. Com., U.S.N.R.

Walter, R.' M. Asst. Prof., New Jersey Coll. for Women, Rutgers Univ., New Brunswick, N.J.

Walton, Dr. L. F. Instr., San Diego State Coll., San Diego, Calif.

Walton, Sister M. Bertrand. Marywood Coll., Scranton, Pa.

Walton, T. O. Prof., Kalamazoo Coll., Kalamazoo, Mich.

Wannemacher, Joseph. 63 Valley Way, West Orange, N.J. U.S.A., Quartermaster Replacement Training Center, Camp Lee, Va.

Ward, J. A. Prof., Delta State Teachers Coll., Cleveland, Miss. Asso. Ground School Instr. U. S. Naval Air Station, Corpus Christi, Tex. 1317 Logan St.

Ward, L. E. Asso. Prof., State Univ. of Iowa, Iowa City, Iowa. 209 Physics Bldg.

Ward, Morgan. Prof., California Inst. of Tech., Pasadena, Calif.

Wardwell, J. F. Asst. Prof., Colgate Univ., Hamilton, N.Y.

Warnock, W. G. Asst. Prof., Univ. of Alabama, University, Ala.

Warschawski, S. E. Asst. Prof., Washington Univ., St. Louis, Mo.

Washburne, A. C. Actuary Emeritus, Berkshire Life Ins. Co., Pittsfield, Mass.

§Wasow, Dr. W. R. 166 E. 91st St., New York, N.Y.

Watkeys, C. W. Prof., Univ. of Rochester, Rochester, N.Y. River Campus.

Watson, Marjorie. Asst. Prof., Huntingdon Coll., Montgomery, Ala. Box 6.

Wayne, Alan. Instr., Rhodes School, New York, N.Y.; Junior High School 184, New York, N.Y. 141-24 78th Road, Flushing, N.Y.

Wear, L. E. Asso. Prof., California Inst. of Tech., Pasadena, Calif. 2247 Lambert Dr.

Weatherburn, C. E. Prof., Univ. of Western Australia, Perth, Australia.

Weaver, Dr. Warren. Dir., Div. of Natural Sciences, Rockefeller Foundation, 49 W. 49th St., New York, N.Y. Chairman, Section D-2, National Defense Research Com.

Webb, D. L. Asst. Prof., Georgia School of Tech., Atlanta, Ga.

Webber, G. C. Asst. Prof., Univ. of Delaware, Newark, Del.

Webber, W. J. Asso. Prof., Univ. of Toronto, Toronto, Ont., Canada.

Weber, Ernst. Prof., Electrical Engineering Dept., Polytech. Inst. of Brooklyn, Brooklyn, N.Y. Official Investigator, Office of Scientific Research and Development.

Webster, M. S. Asst. Prof., Purdue Univ., Lafayette, Ind.

$\dagger$ Wedderburn, J. H. M. Prof., Princeton Univ., Princeton, N.J. Fine Hall.

Wegner, K. W. Prof., Coll. of St. Catherine, St. Paul, Minn.

Wehausen, Dr. J. V. Instr., Univ. of Missouri, Columbia, Mo.

Weida, F. M. Prof., Statistics Dept., George Washington Univ., Washington, D.C.

$\S$ Weil, André. Asst. Prof., Lehigh Univ., Bethlehem, Pa.

Weinberg, I. T. Instr., McMichael Intermediate School, Detroit, Mich. 2611 Gladstone Ave.

Weinstein, Dr. Alexander. Lecturer, Univ. of Toronto, Toronto, Ont., Canada.

Weisner, Louis. Asso. Prof., Hunter Coll., 695 Park Ave., New York, N.Y. 117 W. 197th St., Bronx. 
Weiss, Marie J. Prof., Newcomb Coll., Tulane Univ., New Orleans, La.

Welch, Brother B. A. Asst. Prof., Manhattan Coll., New York, N.Y.

Welch, F. P. Asso. Prof., Mississippi State Coll., State College, Miss. 1216 18th Ave., Meridian, Miss. 1st Lt., U.S.A., Chemical Warfare Service, Huntsville Arsenal, Huntsville, Ala.

Welker, Dr. E. L. Instr., Univ. of Illinois, Urbana, I11. 1207 W. Daniel St., Champaign, Ill.

Wells, Dr. C. P. Instr., Michigan State Coll., East Lansing, Mich.

Wells, Mary E. Prof., Vassar Coll., Poughkeepsie, N.Y.

Wells, N. W. Instr., Springfield Junior Coll., Springfield, Ill.

Wells, V. H. Asso. Prof., Williams Coll., Williamstown, Mass. 3 Chapin Ct.

Welmers, E. T. Asst. Prof., Michigan State Coll., East Lansing, Mich.

Wente, Irene L. Asst. Prof., South Dakota State Coll., Brookings, S.Dak.

Wernick, Dr. William. McKee Vocational High School, St. George, Staten Island, N.Y. 2515 Davidson Ave., Bronx, New York, N.Y.

Wertheimer, Albert. Engineer, Bureau of Ordnance, U. S. Navy Dept., Washington, D.C.

Wescott, M. E. Asst. Prof., Northwestern Univ., Evanston, I11. 1936 Greenwood Ave., Wilmette, I11.

Westbrook, H. S. U.S.A., Army Post Office, San Francisco, Calif.

$\S$ Westergaard, H. M. Dean, Graduate School of Engineering, Harvard Univ., Cambridge, Mass. Pierce Hall.

$\dagger$ Western, Dr. A. E. Windwhistle, Grayshott, Hindhead Surrey, England.

Western, D. W. Instr., Brown Univ., Providence, R.I.

Westfall, W. D. A. Prof., Univ. of Missouri, Columbia, Mo. 11 S. Glenwood Ave.

Westlund, Dr. Jacob. Retired. 301 W. Oak St., West Lafayette, Ind.

de Wet, J. S. Prof., Univ. of Pretoria, Pretoria, South Africa.

$\S$ Wetzel, Marion D. Fellow, Northwestern Univ., Evanston. I11.

Wetzel, R. A. Retired. 218 Tecumseh Ave., Mt. Vernon, N.Y.

Weyl, Dr. F. J. Instr., Indiana Univ., Bloomington, Ind.

Weyl, Hermann. Prof., Inst. for Advanced Study, Princeton, N.J.

Whaples, Dr. George. Instr., Johns Hopkins Univ., Baltimore, Md.

Wheeler, A. H. North High School, Worcester, Mass. 59 Davidson Road.

Wheeler, Anna Pell (Mrs. A. L.). Prof., Bryn Mawr Coll., Bryn Mawr, Pa.

Wheeler, C. H. Prof. and Treas., Univ. of Richmond, University of Richmond, Va.

\$Wheeler, D. B. Instr. in Physics, Occidental Coll., Los Angeles, Calif.

Wheeler, J. J. Asst. Prof., Univ. of Kansas, Lawrence, Kan.

Whelan, A. Marie. Asst. Prof., Hunter Coll., 695 Park Ave., New York, N.Y. 210 E. 68th St.

White, E. F. Asst. Prof., Univ. of Cincinnati, Cincinnati, Ohio.

White, H. S. Prof. Emeritus, Vassar Coll., Poughkeepsie, N.Y. Box 41.

White, Dr. Marion B. Retired. 1949 Woodlyn Road, Pasadena, Calif.

$\S$ White, M. E. 2 Judson St., Hartford, Conn.

White, Dr. Paul. Structural Engineer, North American Aviation, Inglewood, Calif. 3626 W. Adams St., Los Angeles, Calif.

White, R. L. 2122 W. Washington Blvd., Los Angeles, Calif. Ensign, U.S.N.R.

†Whited, Dr. Willis. Retired. 1407 Market St., Harrisburg, Pa.

Whitehead, Dr. G. W. Instr., Purdue Univ., Lafayette, Ind.

Whiteman, Dr. A. L. Instr., Harvard Univ., Cambridge, Mass. Ensign, U.S.N.R., 5529 Osage Ave., Philadelphia, Pa.

Whitford, D. E. Asst. Prof., Polytech. Inst. of Brooklyn, Brooklyn, N.Y.

Whitman, E. A. Asso. Prof., Carnegie Inst. of Tech., Pittsburgh, Pa.

Whitman, Dr. P. M. Instr., Univ. of Pennsylvania, Philadelphia, Pa.

Whitmore, Dr. W. F. Asst. Physicist, Ordnance Lab., Navy Yard, U. S. Navy Dept., Washington, D.C.

Whitney, A. W. Consulting Dir., National Conservation Bureau, 60 John St., New York, N.Y.

Whitney, D. R. 551 Dunbar Ter., Lincolnshire, Crete, Ill. Ensign, U.S.N.R.

Whitney, Hassler. Asso. Prof., Harvard Univ., Cambridge, Mass. Sudbury Road, Weston, Mass. 
$\dagger$ Whittaker, E. T. Prof., Univ. of Edinburgh, Edinburgh, Scotland. 48 George Sq., Edinburgh, 8, Scotland.

$\dagger$ Whittemore, J. K. Asso. Prof., Yale Univ., New Haven, Conn. 45 Lincoln St.

Whyburn, G. T. Prof., Univ. of Virginia, University Station, Charlottesville, Va. Colonnade Club.

Whyburn, W. M. Prof., Univ. of California at Los Angeles, Los Angeles, Calif. 715 Malcolm Ave.

†Widder, D. V. Prof., Harvard Univ., Cambridge, Mass. 572 Widener Lib.

Widder, Dr. Vera A. (Mrs. D. V.). 43 Linnaean St., Cambridge, Mass.

Widmark, L. E. Chief Engineer, Star Electric Motor Co., Newark, N.J. 6 Pen Bryn Road, West Orange, N.J.

$\S$ Wiegmann, N. A. Asst., Univ. of Wisconsin, Madison, Wis. North Hall.

Wiener, Norbert. Prof., Massachusetts Inst. of Tech., Cambridge, Mass.

Wiesenberg, Albert. Columbia Univ., New York, N.Y. 80 Van Cortlandt Park S., Bronx.

Wiggin, Evelyn P. Prof., Randolph-Macon Woman's Coll., Lynchburg, Va. Lecturer, Wellesley Coll., Wellesley, Mass. 26 Horton House.

Wigner, E. P. Prof., Princeton Univ., Princeton, N.J. Fine Hall.

Wilcox, L. R. Asst. Prof., Illinois Inst. of Tech., Chicago, I1l.

Wilczewski, Rev. Joseph. Prof., Marquette Univ., Milwaukee, Wis.

Wilder, C. E. Prof., Dartmouth Coll., Hanover, N.H. 5 Dana Road.

Wilder, R. L. Prof., Univ. of Michigan, Ann Arbor, Mich. Angell Hall.

Wiley, F. B. Prof., Denison Univ., Granville, Ohio.

\$Wilkins, Dr. J. E. Inst. for Advanced Study, Princeton, N.J.

Wilks, S. S. Asso. Prof., Princeton Univ., Princeton, N.J.

Williams, A. R. Asst. Prof., Univ. of California, Berkeley, Calif. 455 Wheeler Hall.

Williams, C. W. Prof., Armstrong Junior Coll., Savannah, Ga.

Williams, Dr. Emily C. (Mrs. W. H.). 7 E. 63d St., New York, N.Y.

Williams, F. G. Prof. and Registrar, Pennsylvania Military Coll., Chester, Pa.

Williams, H. B. Prof. Emeritus, Columbia Univ., 630 W. 168th St., New York, N.Y.

Williams, J. D. Box 587, R.F.D. 10, Phoenix, Ariz. Official Investigator, National Defense Research Com.

Williams, J. E. Dean, Virginia Polytech. Inst., Blacksburg, Va.

Williams, K. P. Prof., Indiana Univ., Bloomington, Ind. 523 E. 3d St.

Williams, L. B. Instr., Hamilton Coll., Clinton, N.Y.

Williams, Dr. Martha H. (Mrs. J. W.). Instr., Univ. of Maryland, College Park, Md. Prince George Apts., Hyattsville, Md.

Williams, W. J. Prof., Baylor Univ., Waco, Tex.

Williams, w. L. G. Prof., McGill Univ., Montreal, Quebec, Canada. Engineering Bldg.

Williamson, C. O. Prof., Coll. of Wooster, Wooster, Ohio. 1141 Beall Ave.

Williamson, John. Asso. Prof., Queens Coll., Flushing, N.Y.

Wilson, A. H. Prof. Emeritus, Haverford Coll., Haverford, Pa.

Wilson, E. B. Prof., School of Public Health, Harvard Univ., Boston, Mass. 55 Van Dyke St.

Wilson, Dr. Elizabeth W. 1 Waterhouse St., Cambridge, Mass.

Wilson, H. A. Prof., Physics Dept., Rice Inst., Houston, Tex. Official Investigator, Office of Scientific Research and Development.

wilson, L. T. Prof., U. S. Naval Acad., Annapolis, Md. 20 Thompson St.

$\S$ Wilson, N. R. Prof., Univ. of Manitoba, Winnipeg, Man., Canada.

Wilson, R. L. Asst. Prof. of Military Science and Tactics, Alabama Polytech. Inst., Auburn, Ala. 1 st Lt., U.S.A.

wilson, Dr. Wilfrid. Asso., Univ. of Illinois, Urbana, I11. 362 Math. Bldg.

Wilson, W. A. Prof., Yale Univ., New Haven, Conn. 1960 Chapel St.

Wilson, W. H. Asso. Dean, Coll. of Arts and Sciences, Univ. of Florida, Gainesville, Fla. 2227 University Station.

†Wilton, J. R. Prof., Univ. of Adelaide, Adelaide, Australia.

Winger, R. M. Prof., Univ. of Washington, Seattle, Wash.

Wintner, Aurel. Asso. Prof., Johns Hopkins Univ., Baltimore, Md. 
Winton, L. S. Asso. Prof., North Carolina State Coll., Raleigh, N.C. 5548 State College Station.

Wirth, H. P. Asst. Prof., Coll. of the City of New York, 139th St. and Convent Ave., New York, N.Y.

Wise, Dr. W. H. Bell Telephone Labs., 463 West St., New York, N.Y.

Witmer, E. E. Asst. Prof., Univ. of Pennsylvania, Philadelphia, Pa. Randall Morgan Lab.

Wolf, Dr. František. Instr., Univ. of California, Berkeley, Calif.

Wolf, Louise A. Asst. Prof., Univ. of Wisconsin at Milwaukee, Milwaukee, Wis. 3700 S. 116th St., West Allis, Wis.

Wolfe, Herbert. Brooklyn Coll., Brooklyn, N.Y. 314 Pulaski St.

Wolfe, H. E. Asso. Prof., Indiana Univ., Bloomington, Ind. 812 S. Fess Ave.

Wolfe, Dr. Jack. Instr., Brooklyn Coll., Brooklyn, N.Y.

Wolfe, R. S. Instr., Northwestern Univ., Evanston, I11.

Wolfenden, H. H. Consulting Actuary and Statistician. 182 Rosedale Heights Dr., Toronto, Ont., Canada.

Wolfowitz, Dr. Jacob. Research Asso., Div. of War Research, Columbia Univ., 401 W. 118th St., New York, N.Y.

Wolkow, Ruth. Barret Junior High School, Louisville, Ky. 2114 Bonnycastle Ave.

Wong, B. C. Asst. Prof., Univ. of California, Berkeley, Calif. 1933 Grant St.

§Wong, Dr. Y. K. Research Asst., Univ. of Chicago, Chicago, Ill.

Wood, Frederick. Dean, Coll. of Arts and Sciences, Univ. of Nevada, Reno, Nev.

Wood, F. E. Asso. Prof., Northwestern Univ., Evanston, I11.

Wood, Dr. H. A. Applied Mathematician, Vought-Sikorsky Aircraft, Stratford, Conn. 1843 Elm St.

Woodard, D. W. Prof., Howard Univ., Washington, D.C. 127 W St. N.W.

Woodbridge, Dr. Margaret Young (Mrs. D. E.). Instr., Brooklyn Coll., Brooklyn, N.Y. 169 Columbia Heights.

Woodbury, M. A. 248 University St., Salt Lake City, Utah. $2 d$ Lt., U.S.A., Air Corps, Colorado Springs, Colo.

Woods, F. S. Prof. Emeritus, Massachusetts Inst. of Tech., Cambridge, Mass. 123 Sumner St., Newton Centre, Mass.

Woods, Roscoe. Asso. Prof., State Univ. of Iowa, Iowa City, Iowa. 210B Physics Bldg.

Woolson, J. R. Asst. Seismologist, United Geophysical Co., 1255 E. Green St., Pasadena, Calif.

Worth, C. R. Asst. Prof., Rutgers Univ., New Brunswick, N.J. Lt., U.S.N.R., U.S. Naval Acad., Annapolis, Md.

Worthington, Euphemia R. Asst. Prof., Univ. of California at Los Angeles, Los Angeles, Calif.

Wray, Dr. W. D. Instr., Williams Coll., Williamstown, Mass. U. S. Navy Dept., Washington, D.C. Box 375, R.F.D. 2, Alexandria, Va.

Wrench, Dr. J. W. Instr., George Washington Univ., Washington, D.C. 3604 Massachusetts Ave. N.W. Chief Computer, National Defense Research Com.

Wright, C. B. Asso. Prof., East Texas State Teachers Coll., Commerce, Tex.

Wright, $\mathrm{H}$. N. Pres. and Prof., Coll. of the City of New York, New York, N.Y. 3900 Greystone Ave., Bronx.

Wundheiler, Dr. Alexander. Instr., Physics Dept., Coll. of the City of New York, 139th St. and Convent Ave., New York, N.Y. 452 W. 144th St.

Wyckoff, J. F. Instr., Trinity Coll., Hartford, Conn.

Wylie, C. R. Asst. Prof., Ohio State Univ., Columbus, Ohio.

Wyman, Dr. Max. No address.

Wynne, W. M. 149 68th St., Brooklyn, N.Y. Com., U.S.N.

Yagi, Fumio. Massachusetts Inst. of Tech., Cambridge, Mass. Graduate House. Yahner, Rev. C. G. St. Vincent Archabbey, Latrobe, Pa.

Yanney, B. F. Prof. Emeritus, Coll. of Wooster, Wooster, Ohio. 354 E. Bowman St.

Yanosik, G. A. Asso. Prof., New York Univ., University Heights, New York, N.Y. 52 Greenvale Ave., Yonkers, N. Y. 
Yarbrough, H. M. Prof., Western Kentucky State Teachers Coll., Bowling Green, Ky. R.F.D. 4.

Yeaton, C. H. Prof., Oberlin Coll., Oberlin, Ohio. 189 Forest St.

York, Marie. 1075 Bryant Ave., Bronx, New York, N.Y.

Yoshida, Dr. Minoru. Box 665, Tokyo Central, Tokyo, Japan.

Young, J. W. A. Asso. Prof., Univ. of Chicago, Chicago, I1l. 5422 Blackstone Ave.

Young, Mabel M. Prof. Emeritus, Wellesley Coll., Wellesley, Mass. 6 Norfolk Ter.

Young-Woodbridge, Margaret. See Woodbridge, Margaret Young.

Youngs, J. W. T. Asst. Prof., Purdue Univ., Lafayette, Ind.

Yowell, Dr. E. I. Dir. of Observ. and Prof., Úniv. of Cincinnati, Cincinnati, Ohio. 3127 Griest Ave.

Zariski, Oscar. Prof., Johns Hopkins Univ., Baltimore, Md.

Zeldin, S. D. Asst. Prof., Massachusetts Inst. of Tech., Cambridge, Mass.

Zilmer, D. E. Research Engineer, Lockheed Aircraft Corp., Burbank, Calif. 11165 Morrison, North Hollywood, Calif.

Zimmer, J. W. Instr., Michigan State Coll., East Lansing, Mich.

Zimmerberg, H. J. Asst., Univ. of Chicago, Chicago, Ill.

Zippin, Dr. Leo. Instr., Queens Coll., Flushing, N.Y. 1170 E. 24th St., Brooklyn, N.Y.

Zobel, Dr. O. J. Bell Telephone Labs., 463 West St., New York, N.Y.

Zorn, Max. Asso. Prof., Univ. of California at Los Angeles, Los Angeles, Calif. 10589 Kinnard Ave.

Zuckerman, Dr. H. S. Instr., Univ. of Washington, Seattle, Wash.

Zwillinger, Max. Senior Engineer, Optical Shop, Navy Yard, U. S. Navy Dept., Washington, D.C.

Zygmund, Antoni. Asso. Prof., Mt. Holyoke Coll., South Hadley, Mass. 61 College St.

Number of Members, September, 1940 (including 204 nominees of Institutional Members)

Members admitted, 1941-1942

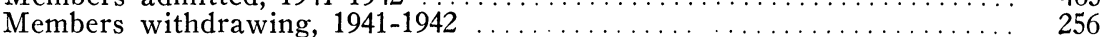

Number of Members, September, 1942 (including 227 nominees of Institutional Members) ..................................... 2523

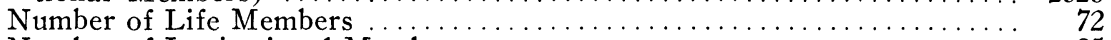

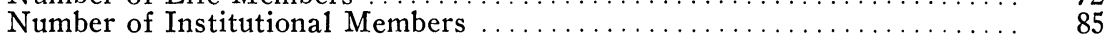

